

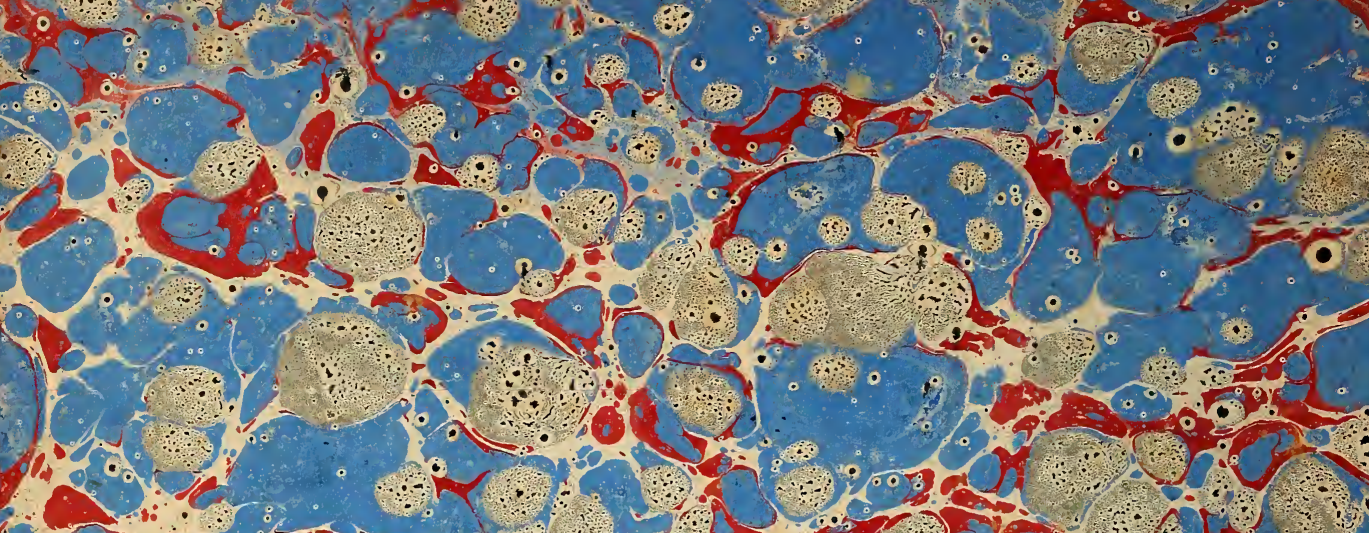

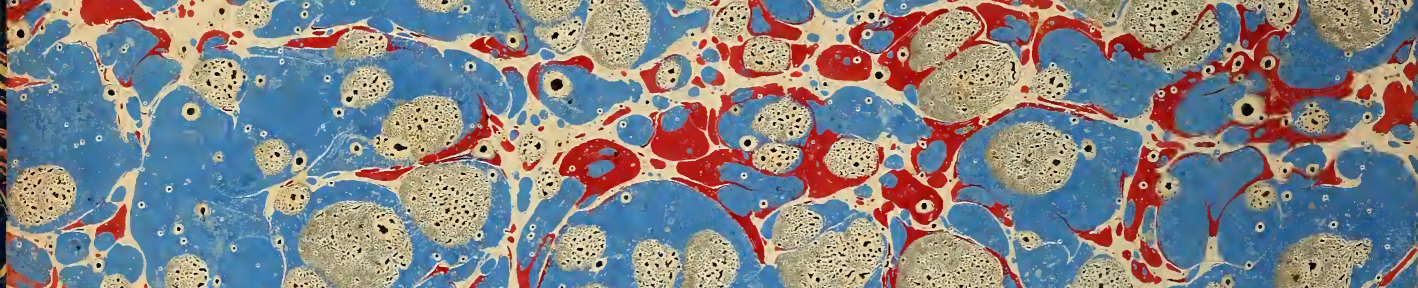
ate

25 $+6$

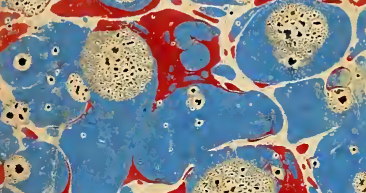

:

3 1.

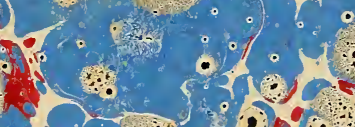
42. a. $=5$

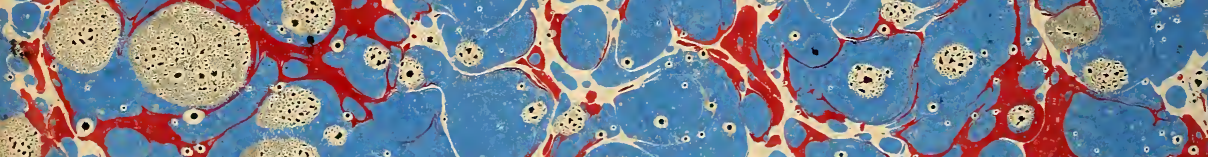

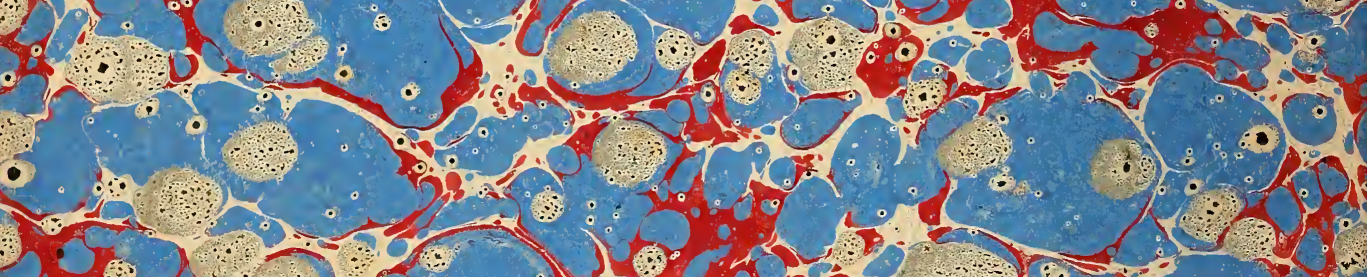
$2020 \% 202^{2}=0$

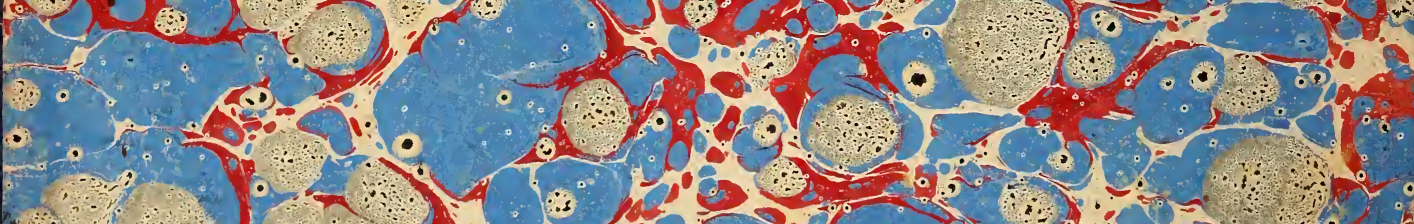







\section{AUG.DE RIDDER \\ Eikenstraat, 5 ANTWERPEN}

\section{LE CONGO BELGE}





\section{LE}

\section{CONGO BELGE}

INITIATION A LA COLONISATION NATIONALE

PAR

J. BERTRAND

Professelr de Géographie

a l'École Normale de Charleroy
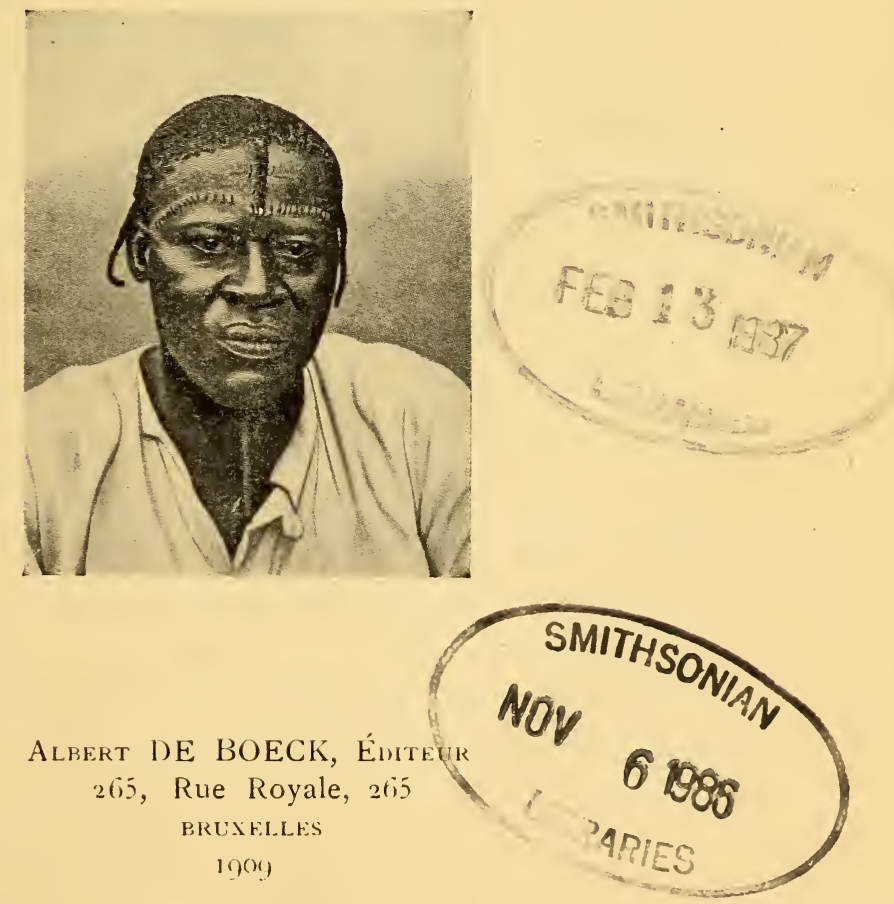



\section{PREFACE}

Nous présentons au public un petit opuscule dont la matière, condensée, devait primitivement constituer un chapitre d'un cours élémentaire de géographie moderne. Nous sommes assurés que beaucoup de questions traitées dans ce petit ouvrage comporteront d'ici peu des résolutions plus amples si pas différentes. Etant donné l'esprit d'améliorisme que nous avons rencontré en haut lieu, nous croyons pouvoir affirmer l'imminence d'heureuses réformes au Congo. Notre opuscule est une esquisse générale, un aperçu géographique. A la demande de plusieurs personnes d'enseignement colonial, nous nous sommes décidé à offrir aujourd'hui ce petit livre d'initiation. Nous demandons au public autant d'indulgence à nous lire que nous avons mis de bonne volonté à écrire.

L'illustration de cette brochure, quoique travaillée et recherchée avec soin, est nécessairement insuffisante, aussi engageons-nous très vivement nos lecteurs, petits et grands, à visiter avec attention les serres du jardin botanique de Bruxelles, du parc royal de Laeken et surtout le Musée Colonial de Tervueren. 
L'orthographe géographique que nous avons adoptée pour des raisons d'ordre pédagogique diffère de l'orthographe officielle. C'est que seule la prononciation nous a guidé dans la transcription française des noms. Exempless :

\section{Orthographe officielle Prononciation \\ Ubanghi. . . . . Oubanghi \\ Etshutshu . . . . Étchouchou \\ Lubilash . . . . Loubilach \\ Dungu . . . . . Doungou \\ Etc...}

Nous avons adopté l'aspect phonétique pour amoindrir le doute au sujet d'une prononciation singulièrement élastique d'ailleurs et souvent très difficile à saisir.

Nous remercions de tout cœur ceux qui ont bien voulu nous aider dans notre documentation, notamment M. A.-J. Wauters, directeur du Mouvement géographique et notre ami Ch. Lemaire, explorateur, qui nous a ouvert ses manuscrits et ses collections.

J. BERTRAND.

Bruxelles, 20 mai 1909. 


\section{TABLE DES MATIĖRES}

la Colonisation . . . . . . . . . . . . . . . . 1

La Reconfaissance de l'Afrique équatoriale. - La Constitution de L'Etat indépendant du Congo. - La Colonie belge du Congo . . . . . . . . . . . . . . 3

Le Milieu naturel . . . . . . . . . . . . . 25

I. Position astronomique et situation géographique . 25

1I. Le sol . . . . . . . . . . . . 27

Le relief . . . . . . . . . . . . . 27

La structure et la nature du sol . . . . . . . . 33

III. Le climat . . . . . . . . . . . . . . 34

La température. . . . . . . . . . . . . . 35

Les saisons . . . . . . . . . . . . . . . 38

IV. Les rivières . . . . . . . . . . . . . . 44

Le Congo . . . . . . . . . . . . . . . 44

Le Nil . . . . . . . . . . . . . . . . . 53

Le régime des eaux. . . . . . . . . . . . . 54

V. La végétation . . . . . . . . . . . . . 56

VI. Les animaux. . . . . . . . . . . . . . 68

L'Indigène. - Son État de culture. - La Société indigène. 73

\. Densité de peuplement. . . . . . . . . . . . 73

II. Caractères, langues et genre de vie . . . . . 73

III. Le vêtement . . . . . . . . . . . . . . . 81

IV. L'habitation et la famille. . . . . . . . . . . 84

V. L’agglomération. . . . . . . . . . . . . . 86

VI. L'organisation politique et sociale . . . . . . . 86

VII. Les échanges, les marchés, les routes . . . . . 89

VIII. Les croyances et les arts. . . . . . . . . . . 93 
IX. Les tribus. . . . . . . . . . . . . . . . . . 96

$1^{\circ}$ Bantous-Congos de la còte et de la Savane. . $\quad 96$

$2^{\circ}$ Bantous-Congos du centre forestier. . . . . . 97

30 Bantous-Congos de la Savane et de la brousse
ou du sud et du sud-est. . . . . . . .

$t^{\circ}$ Les Nilotes . . . . . . . . . . . . . . 100

$5^{\circ}$ Les Négrilles. . . . . . . . . . . . . . 100

$6^{\circ}$ Les Sangs-Mêlés . . . . . . . . . . . . 101

X. Meilleurs que nous ou pires. . . . . . . . . . 103

Le Gouvernement colonial. - Régime politique, administra-

TIF, JUDIGIAIRE, FINANGIER . . . . . . . . . . . . 105

I. Le gouvernement . . . . . . . . . . . . . . 105

II. La population blanche. . . . . . . . . . . . 108

III. Les cultes. . . . . . . . . . . . . . . . . 110

IV. La force publique. . . . . . . . . . . . . . 110

V. La justice . . . . . . . . . . . . . 110

VI. Les finances . . . . . . . . . . . . . . . . . . . 110

La dette. . . . . . . . . . . . . . . 110

Les impôts. . . . . . . . . . . . . ... . 111

La monnaie . . . . . . . . . . . . . . 112

VII. Le régime foncier. . . . . . . . . . . . . . . . . 112

La Situation économeue de la Colonie . . . . . . . . . . 117

I. Exploitation forestière et agricole . . . . . . . . 117

Les bois. . . . . . . . . . . . . . . . 117

Les huiles. . . . . . . . . . . . . . . . 119

Les gommes et résines . . . . . . . . . . . 120

Les textiles . . . . . . . . . . . . . . . 123

Les cultures alimentaires . . . . . . . . . . . 124

Les plantes médicinales et aromatiques . . . . 127

Les animaux. . . . . . . . . . . . . . . 128

L'élevage . . . . . . . . . . . . 130

II. Les gîtes minéraux et les roches . . . . . . . . . 132

III. Le mouvement commercial . . . . . . . . . . 134

IV. Les voies de communication . . . . . . . . . . 136

Comment on arrive au Congo . . . . . . . . . 138

Comment on circule au Congo . . . . . . . . 138

V. La main-d'ouvre . . . . . . . . . . . . . . . 142

L'Avenir de la Colonie . . . . . . . . . . . . . 145 


\title{
TABLE DES FIGURES
}

\author{
Les reproductions photographiques sont indiquées en caracteres \\ ordinaires; les cartes et diagrammes en caractères italiques.
}

F1G. I. La disposition du relief africain; i $\dot{a} 80.000 .000$. . . . . . . . . 4

3. David Livingstone . . . . . . . . . . . . 7

3. Henry .I. Stanley . . . . . . . 9

+. Le partage de l'Afrique; i à $80.000 .000 \quad$. . . . . . . . . . . . . $\quad 15$

5. George Grenfell. . . . . . . . . . . . . . . 17

○. Nlexandre Delcommune . . . . . . . . . . . . . . . . 19

7. La deconverte de l'Afrique 22

8. Etendues comparées de la Belgique et de son domaine colonial; 26

9. Superficies comparées . . . . . . . . . . . . . . . . . 27

ro. Profil de l'Afrique par l'équatenr; i à 25000.000 . . . . . . . $\quad 29$

II. La disposition du relief congolien; i à 25.000 .000 . . . . . . . . 30

12, 13, r+. Trois profils du relicf congolien; i à rn.000.00o . . . . . . 31

15. Le pays des volcans au nord du lac Kivou . . . . . . . . 33

I6. Esquisse approximative de la géologie ilu Bassin dü Congo; 34

17. Diagramme comparatif de la marche ammelle de la température $\quad 36$

18. Variation diurne de la temperature au Congo et en Belgique . . $\quad 37$

19. Marche ammelle moyenne des plnies i Banana . . . . . $\quad 39$

20. Marche anmelle moyenne des pluies a Kimonenza . . . . 40

21. Marche amuelle moyenne des pluies i Eala . . . 40

22. Harche ammelle moyenne des pluies i Loukaf, $u$. . . . . 41

23. Marche anmelle moyenne des pluies i Mono. 42

24. La répartition annulle de la pluie; i à $25.000 .000 . \quad$. . . . . 43

25. Etcudues comparés des réseanx hivlrographigne's du Congo 45

26. Longuemrs comparées de's six plus grands flente's du monde' . 46

27. Haute plaine du Loualaba . . . . 48

28. La haute plaine du Loualaba et l'entrie diu gonlot du N'silou; ;

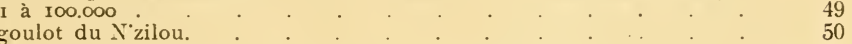

29. Le goulot du N'zilou.

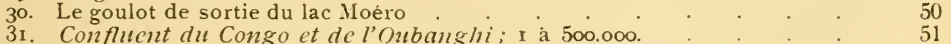

32. Stanley-Pool . . . . 51

33. Stanley-Pool; i à 500.000 . . . . . . 52

34. Le Bas-Congo; i à r.000.000. . . . . . . . . 53

35. La rivière Tchopo (chutes) . . . . . . . . . . . . 54

36. Une éclaircie dans la forét dense . . . . . . . . . . . . . 57

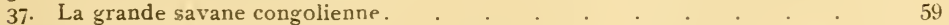

38. La savane à Baobab . . . . . . . . . . . . . . . . . . 60

39. La forêt en galerie . . . . . . . . . . . . . 61

to. De la savane à la brousse. . . . . . . . . . . . . . . 62

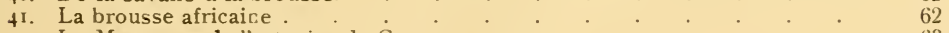

2. La Mangrove de l'estuaire du Congo : . . . . . . . . $\quad 63$ 
43. Schéma de la distribution du tapis végétal

44. Palmiers à l'huile

45. Liane ficus enserrant le tronc d'un Capoquiei

46. La forêt du Haut-Katanga.

47. Une bananeraie .

48. Une termitière

49. La mouche tsetsé

5o. Répartition de la population; esquisse approximative; I à 25.000 .000

5. Un Bantou

52. Katanga. Village Ka-Mapembwe

53. Femmes préparant la farine de maïs

54. Pêcheries dans les rapides du fleuve

55. Piège à gibier

56. Femmes fabriquant des nattes

57. Vanniers

59. Tréfilerie de cuivre

6o. Haut-fourneau indigène au Katanga

6r. Chef paré

62. Chef tatoué

63. Coiffures d'hommes

64. Femme d'un chef du Katahga (coiffure)

65. Huttes rectangulaires

66. Huttes sur pilotis (Ouellé)

67. Maison arabisée à Lofo

68. Type de village rangé

69. Village du Katanga: Tchiamakélé

7o. Scène de marché à Lokandou .

7r. Marché des Mangbettous à Nyangara

72. Un fétiche

73. Un orchestre

74. Un soliste

75. Homme batéké .

76. Jeune homme bangala

77. Pirogue bangala sur le Congo

78. Jeune fille oubanghi .

79. Piroguiers sur le haut Congo

8o. Indigènes de l'Ouroua

81. Troupeau d'un chef indigène du Bahr-el-Ghazal

82. Femme Azandé

83. Chef Mangbettou

84. Panorama d'une station : M'Pwéto en 1898

85. Vue générale d'une station militaire : Doungou en I9o3

86. Campement de la Mission scientifique du Katanga

87. Intérieur d'un poste de l'Etat : Faradj.

88. Type d'une habitation pour Européen

89. Esquisse de l'appropriation foncière : I à 25.000 .000

9o. Arachide

9I. La récolte du caoutchouc

92. L'expcrtation du caoutchouc de 1887 à 1907

93. Maniuc

94. Un caféier d'Afrique.

95. Fleur et fruit du caféier .

96. Défenses d'un éléphant

97. L'exportation de l'ivoire

98. Emploi des éléphants aux Indes

99. Le commerce spécial du Congo

Ioo. Gare de Léopoldville en Igo5.

IoI. L'Afrique politique; les voies de communication; i à 80.000 .000

I02. Un sternwheel

I03. La route de roulage près de Lousambo 


\section{LA COLONISATION}

C'est dans les pays tempérés que la haute civilisation a pu s'épanouir grâce à l'effort continu que la nature a imposé à l'homme. Le développement de la civilisation entraînant une augmentation des besoins, l'activité humaine est vivement sollicitée par l'exploitation de pays nouvellement découverts et naturellement riches. C'est cette exploitation qui sera la colonisation; l'exploitant sera le colon; le pays exploité la colonie. Le climat de ces pays nenfs permettra ou ne permettra pas à l'homme des régions tempérées d'aller s'y installer à demeure avec sa famille. Dans le premier cas le peuplement par des colons sera possible et la colonie sera dite colonie de peuplement; c'est le cas de la Nouvelle Zélande et du Transvaal par exemple. Dans le second cas, l'homme des régions tempérées ne peut séjourner qu'un certain temps sous le climat colonial, il organise l'exploitation de la terre en se servant de travailleurs indigènes; la colonie est alors dite colonie d'exploitation. C'est jusqu'à présent et ce sera longtemps encore, toujours peut-être le cas du Congo.

La colonie de peuplement peut être fondée par des immigrés originaires d'un même pays ou de pays différents. Elle peut devenir pays indépendant comme l'était le Transvaal, comme l'est la République Argentine. La colonie d'exploitation relève toujours d'une puissance extérieure dite Métropole. Exemples : les colonies de l'Afrique équatoriale parmi lesquelles le Congo. Celui-ci, en effet, a toujours relevé d'une 
autorité ayant son siège dans un pays tempéré de haute civilisation.

La colonisation ne doit pas avoir pour but l'enrichissement direct de la métropole par l'exploitation des produits et des indigènes de la colonie, mais bien l'apport sur le marché du monde d'une plus grande abondance de produits. Il en résulte un accroissement dans le commerce de la métropole, avantage que vient augmenter celui de débouché à la surpopulation dans le cas des colonies de peuplement.

Un des buts de la colonisation, et non le moindre, devrait être l'initiation et l'élévation de l'indigène à un degré supérieur de culture. Le plus haut effet de la colonisation sera atteint lorsque l'exploitation entière reposera sur cette bonne initiation. Ces circonstances peuvent se réaliser dans les colonies d'exploitation autant que dans les colonies de peuplement.

A l'exception de l'Abyssinie et de la République nègre de Libéria, pays indépendants, l'Afrique intertropicale entière peut être classée parmi les colonies d'exploitation. 


\section{LA RECONNAISSANCE DE L'AFRIQUE ÉQUATORIALE. - LA CONSTITUTION DE L'ÉTAT INDÉPENDANT DU CONGO. - LA COLONIE BELGE DU CONGO.}

La disposition du relief africain qui se présente sur presque toute l'étendue des côtes sous l'aspect d'une barrière étagée en terrasses a toujours rendu difficile l'accès du continent. Les vallées qui s'ouvrent à l'Océan sont barrées par ’es exhaussements des terrasses côtières que les rivières et fleuves franchissent en de longues séries de chutes et de rapides. Le climat inhospitalier des étroites plaines maritimes et en général de toute l'Afrique accentua les difficultés de pénétration. Mais l'actıvité inlassable des Européens en quête de débouchés finit par avoir raison de ces obstacles et les barrières bordières une fois franchies, la reconnaissance de l'Afrique devait progresser très rapidement.

Jusqu'à la fin du $\mathrm{XVIII}^{\ominus}$ siècle, nous ne connaissions de l'Afrique que la bordure côtière. Il est vrai que déjà des Français s'étaient engagés dans le bassin du Sénégal, que les Boers (I) s'étaient fixés en colons agriculteurs libres sur

(1) En 1652, bien avant la conquête du Cap par les Anglais, des colons hollandais en petit nombre allèrent s'y établir. Quelques années après, à la fin du xvir ${ }^{\ominus}$ siècle, leur nombre s'accrût considérablement par l'arrivée de protestants français chassés de leur pays par le malheur national que fut la révocation de l'Edit de Nuntes (promulgué par Henri IV en 1598, révoqué par Louis XIV en 1685). 
les territoires du Cap et qu'en quelques autres points tels que l'Abyssinie et la Zambésie, l'expansion du christianisme ou les efforts de missionnaires chrétiens pour la conversion des

\section{LA DISPOSITION DU RELIEF AFRICAIN}

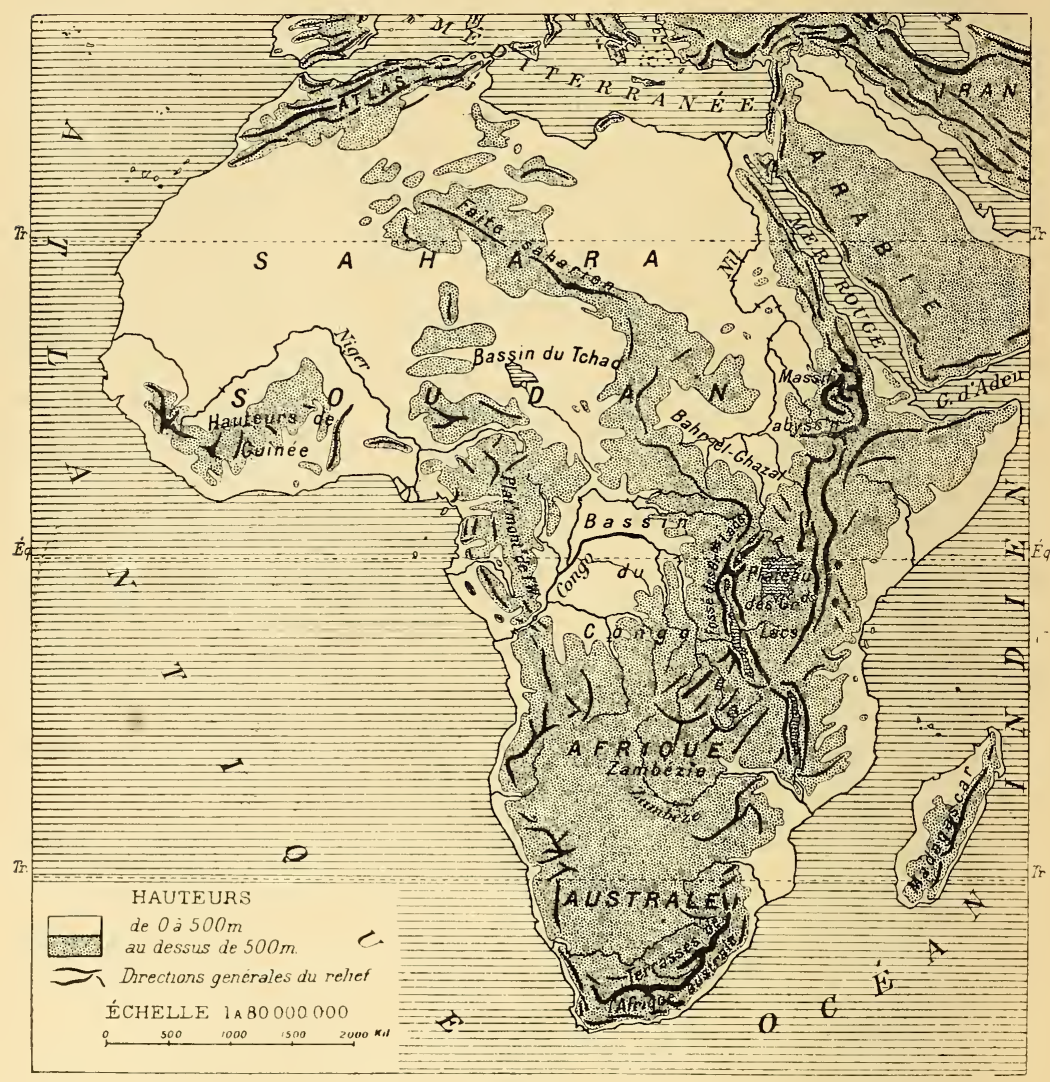

Fig. I

indigènes ne nous avaient pas laissés dans une ignorance totale au sujet de ces régions. Néanmoins le vaste intérieur du continent africain restait mystérieux et c'est plus tard, 
vers I 800 , que les premières tentatives sérieuses de pénétration se réalisèrent.

De I788 à I830, l'allure générale du Niger fut reconnue. De I 800 à I 823 , on s'appliqua à reconnaître le Nil et l'on réussit à remonter jusqu'au Nil blanc et au Nil bleu (fondation de Khartoum). De I8 I 5 à I843, les migrations des Boers fuyant la domination anglaise eurent pour effet la reconnaissance d'une partie considérable de l'Afrique australe. Mais c'est après 1850 que le grand effort fut donné : de I849 à 1874, le Sahara est pénétré et traversé de Tripoli à la Bénoué et au Niger par le Tchad; il est reconnu dans sa partic orientale entre le 'I'chad et l'Égypte.

Le problème du Congo se lia naturellement à celui du Nil. Les deux voies obstruées par des rapides et des chutes ne furent point remontées jusqu'à leurs origines. Le Congo lui-même fut reconnu par l'aval à 300 kilomètres seulement de son embouchure, au delà d'Isanghila, un peu au-dessus des premiers rapides rencontrés (Tuckey, officier anglais, I8I6). Les deux bassins furent pris à revers. La voie d'accès fut celle de la côte orientale avec Zanzibar comme point d'attache. Les têtes du Nil furent découvertes, la région des grands lacs explorée (Burton et Speke au Tanganika, I857, Speke au Victoria, 1858). Les confins septentrionaux du bassin congolien furent reconnus par Schweinfurth depuis les pays du haut Nil jusqu'à l'Ouellé moyen. Du côté méridional, la région supérieure congolienne est englobée dans le vaste domaine des explorations de Livingstone à travers toute l'Afrique australe. Pendant 30 ans, il va du Zambèze à l'Atlantique (Saint-Paul de Loanda), de l'Atlantique à l'Océan Indien (Quilimané), du Zambèze inférieur au lac Nyassa et à Zanzibar, de Zanzibar au haut-Congo (Nyangwé), de Nyangwé au Tanganika et au . Bangwélo non loin duquel il meurt. Les mêmes régions du sud-est et du sud du Congo furent aussi parcourues de 1873 à 1875 
par l'anglais Cameroon, parti de Zanzibar. Il reconnaît la Loukouga, affluent du Tanganika, est empêché de descendre le maître fleuve en aval de Nyangwé et se reporte vers l'Ouroua, le Katanga, le Lounda et l'Atlantique.

Sans affirmer que le fleuve de Nyangwé était le haut Congo - il n'avait pour cela pas assez d'éléments Livingstone s'en doutait bien. Il avait déjà résolu la question du Zambèze et avait selon toute probabilité formé intérieurement le projet de revenir à Nyangwé pour tenter la percée de l'inconnu. Cet honneur devait échoir à Stanley (I874 à 1877)

La figure de David Livingstone domine la série héroïque des explorateurs africains. Barth, allemand, qui de 1850 à I 855 posa les jalons les plus sûrs de la connaissance du Sahara et du Soudan, Nachtigal, allemand, qui de 1869 à I 874 explora des régions sahariennes où personne n'a pénétré depuis, Speke, anglais, dont le labeur fut le plus effectif dans la découverte des sources niliennes, Cameroon, dont nous avons parlé, Stanley, qualifié le plus grand explorateur de l'époque contemporaine et dont les exploits tiennent de la légende, sont les têtes les plus marquantes parmi ceux qui, au cours du XIXe, dévoilèrent la terre d'Afrique.

Livingstone, Stanley, individualités opposées.

Le premier, écossais, missionnaire, de savoir profond, explorateur par vocation, civilisateur type, porteur de la honne parole, guidé par une conception rigoriste de ses devoirs et des droits d'autrui, se donnant complètement à ces pauvres indigènes dans lesquels il respecte et aime l'homme, à ces pauvres indigènes pour lesquels il mourut, entouré de leurs soins émus ( ${ }^{\text {er }}$ mai i 873, Tchitambo, sud du lac Bangwélo). Exemple unique : ils avaient tant aimé leur grand ami qu'ils voulurent lui épargner, dans une vie future à laquelle ils croyaient, la souffrance de l'éloignement de sa terre natale et de ses proches; ils lui rendirent l'hommage suprême 
de rapporter sa dépouille à la côte africaine afin que les blancs la fissent transporter dans son pays. A Tabora, ils rencontrèrent Cameroon venu à la recherche de l'illustre

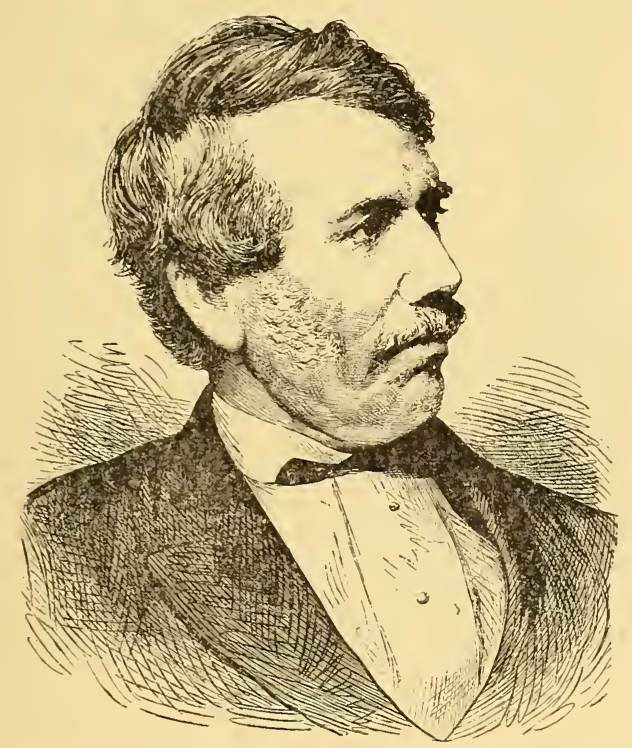

Fig. 2. - David LIVINGSTONE, missionnaire et savant écossais, né à Blantyre, en Écosse (1813-1873). Resta plus de 30 ans en Afrique. De 18+9-1851 : Kolobeng (Betchouana), Ngami, Kalahari, Zambèze ; - I852-1856: du Zambèze à Saint-Paul-de-Loanda et retour par les chutes Victoria et Quilimané; - 1858-1864: Zambèze inférieur, lacs Chirwa et Nyassa ; - I866-1873: Zanzibar, lac Nyassa, Tchambézi, Bangwélo, Louapoula, Moéro, Loualaba Nyangwé, Tanganika, Oudjidji (rencontre de Stanley, 1871), Tanganika, Bangwélo. Il meurt à Tchitambo au sud du Bangwélo, le ${ }^{\text {er }}$ mai 1873. - Ouvrages de Livingstone: Explorations dans l'intérieur de l'Afrique australe et voyages à travers le continent de Saint-Paul-de-Loanda a l'embouchure du Zambèse de $18+0$ à 1856. I vol. 688 p. Trad. Loreau. Hachette, 1877; - Narration d'une expédition au Zambèze et à ses tributaires et déconverte des lacs Chirwa et Nyassa. Trad. Loreau. Paris. 1866; - Dernier journal de Livingstone, relatant ses explorations et découvertes de 1866 a 1873, suivi du récit de ses derniers moments et du transport de ses restes. 2 vol. 800 p. Trad. Loreau. Hachette, 18;6.

explorateur. Ils en conservèrent le souvenir vénéré et vivace qu'ils transmirent à la génération indigène actuelle. Celle-ci évoque encore sa mémoire avec un peu de l'amour qu'il 
donna à ses frères noirs et avec beaucoup de l'admiration dont il fut l'objet de la part de ceux qu'il approcha.

Stanley, anglais américanisé, journaliste, homme d'action et d'espace, de coup d'œil sûr et de volonté claire, d'une confiance en soi illimitée, projetant et isolant son but comme sur un écran et le poursuivant avec une persévérance et une force d'action prodigieuses sans que rien puisse l'en détourner, mettant tout au service de la réalisation de la tâche qu'il s'est imposée, considérant l'homme blanc ou noir, placé sous son autorité, exclusivement comme un moyen dont il disposera absolument dans la lutte qu'il a livrée. Son gigantesque raid de I I,663 kilomètres en 999 jours, la plus célèbre des expéditions africaines, le place parmi les premiers des grands découvreurs. "Après ce prodigieux exploit, qui témoigne chez son auteur d'une audace et d'une énergie merveilleuses, d'une persévérance indomptable, d'un ascendant moral extraordinaire et d'un génie militaire de premier ordre, il ne restait plus qu'à contrôler dans ses détails l'œuvre accomplie,..." " (Elisée Reclus). Nous eussions préféré ignorer ce génie militaire se révélant dans des combats du fusil perfectionné contre la flèche et la lance. Trop homme d'action pour essayer de pénétrer la psychologie simple de l'indigène africain vierge de tout contact avec le blanc, avec l'homme habillé, le mo'n'délé, dominé par la pensée volontaire du but à atteindre, Stanley écrasa les obstacles vivants ou inanimés. Ce qui était secondaire pour lui était essentiel pour Livingstone : communiquer à l'indigène la confiance par la persuasion. Stanley traverse, Livingstone civilise. Stanley commande, Livingstone persuade. Le but de celui-ci est l'homme; le but de celui-là est l'action et pour l'accomplir il livre 32 combats meurtriers, il fait tomber les hommes qui se dressent devant lui comme il fait sauter les rocs qui barrent sa route. Il est Boula-Matari, le briseur de rochers, et a laissé dans l'esprit du nègre fasciné le souvenir d'un géant ter- 


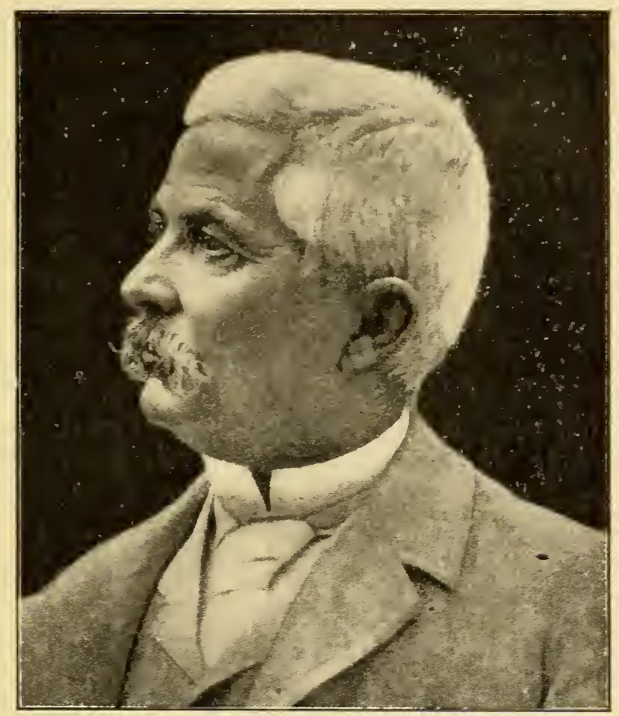

Fig. 3. - John Rowland dit Henry Morton STANLEY (18+1-I904), anglais?du pays de Galles, américanisé, journaliste. Le plus grand explorateur d’Afrique; fit quatre explorations : $I^{\circ}$ à la recherche de Livingstone qu'il retrouva et secourut; le quitta au Tanganika et rentra en Europe en $1873 ;-2^{\circ} 17$ novembre $187+-9$ avril $18 \%$, mission scientifique subventionnée par le New-York Herald et le Daily Telegraph, expédition africaine la plus célèbre; Bagamoyo (Oc. Ind.), lac Victoria, Tanganika, Kasongo, Nyangwé, StanleyFalls, Stanley-Pool, Ntamo (Léopoldville), chutes Livingstone, Boma. Résultat mémorable : découverte et ouverture au monde du grand fleuve Congo $;-3^{\circ}$ 18/9-1884, pour le compte du roi Léopold II, exploration systématique du bassin du Congo $-4^{\circ}$ r887-r889, expédition anglaise au secours d'Emin-Pacha ( $\mathrm{D}^{\text {r }}$ Schnitzler), gouverneur égyptien de la province Equatoria, bloqué sur le Nil par les Mahdistes (jonction au lac Albert le 17 février I89g). Ouvrages de Stanley : Comment j"ai retrouvé Livingstone, I vol. 600 p. Hachette, I\&8o. - A travers le continent mystérieux, descente du fleuve Congo, 2 vol. ro52 p, Hachette, 18;9; - Cinq années au Congo, I vol. 776 p. Bruxelles, Institut national de géographie, 1885; - Dans les Tënèbres de l'Afrique. Recherche, délizrance ct retraite d'Emin-Pacha, 2 vol. Iooo p. Hachette, 1890.

(Reprod. du portrait de Stanley publié par Su H. Johnston dans The Nile Quest.Alston Rivers. Londres.) 
rible. Renom mondial, tâché de sang humain, auquel nous préférons la gloire pure du nom de Livingstone.

Quoiqu'il en soit, saluons la force de Stanley. Dans son premier voyage, il retrouva et accompagna Livingstone dans une reconnaissance au Tanganika. En seconde expédition, il s'engagea sur la terre africaine par Bagamoyo, fit la circumnavigation du lac Victoria, reconnut la tête la plus lointaine du Nil (Kagera, affluent du lac Victoria), découvrit le lac Albert-Édouard, fixa la ligne côtière du Tanganika, gagna . le Congo à Kasongo et perça la terre inconnue, suivant le fleuve jusqu'à son embouchure.

"Commissionné par deux grands journaux : le New York Herald et le Daily Telegraph, le voyageur américain part de Bagamoyo, à la côte orientale, le 17 novembre 1874, et gagne par la route de Speke le lac Victoria, dont il accomplit la circumnavigation. Il découvre ensuite le lac Albert-Édouard (janvier 1876) et fait la reconnaissance complète des rives du Tanganika (11 juin31 juillet).

) A Kasongo, il trouve établi Tippo-Tip, le trafiquant arabe de Zanzibar, qu'il interroge sur les moyens pratiques de reconnaître le cours du Congo en aval et qu'il décide à l'accompagner dans sa tentative hardie.

) Le 5 novembre 1876, les deux expéditions, fortes ensemble de 400 hommes, quittent Nyangwe et pénètrent dans la grande forêt équatoriale. Au prix des plus pénibles efforts, la colonne lutte pendant quatorze jours contre la végétation géante qui l'étouffe et entrave sa marche, et elle atteint le fleuve, déjà décimée par la fatigue et la maladie. Tippo-Tip hésite, Stanley tient bon. Moitié par eau, moitié pédestrement le long de la rive, l'expédition reprend sa marche en avant. Mais de toutes parts surgissent les indigènes en armes. Pour passer, il faut livrer de nombreux combats et voici, en outre, que la petite vérole et la dysenterie se propagent, avec leur lugubre cortège de misères. L'expédition est arrivée au confluent de la rivière Kasuka; il y a cinquante jours qu'elle voit grandir chaque jour les difficultés et les périls de la route. Tippo-Tip déclare renoncer à une tâche qu'il considère comme étant au-dessus des forces humaines.

"Stanley, lui, ne songe pas un instant à abandonner la partie; il 
arrachera son secret au grand fleuve. Ses hommes, dévoués, confiants, et que son ardeur enflamme, achèrent de réunir la flottille d'embarcations nécessaires au voyage et, le 20 décembre, après avoir pris congé des Arabes, l'expédition, forte encore de 150 personnes réparties sur 23 embarcations, s'engage dans l'inconnu mystérieux.

) Le premier obstacle naturel qui arrête la flottille fut la série de rapides à laquelle l'explorateur a donné son nom (StanleyFalls); il fallut vingt jours pour le franchir ou le contourner (6-25 janvier 187i).

»Le $1^{\text {er }}$ février, l'expédition dépassa le confluent de l'Aruwimi, où les guerriers bazoko lui livrèrent un combat en règle. Puis ce fut le tour des Bangala, arec qui elle eut un engagement le 14 . Ce fut le dernier conflit de la vaillante caravane avec les naturels. Le 9 mars, elle passait devant la bouche du Kasaï, arrivait, le 12, au Stanley-Pool et campait au village de Ntamo, qui, cinq ans plus tard, devait échanger son nom indigène contre celui de Léopoldville.

) Les natifs de cette région étaient moins belliqueux que ceux des zones d'amont; mais, au delà du Pool, la nature allait se charger de dresser devant Stanley des obstacles d'un autre genre. Il eut alors à vaincre la colère du fleuve, torrent furieux roulant dans un lit profond, traversant des gorges tortueuses, tombant, écumant de terrasses en terrasses; aussi, l'explorateur mit-il cinq mois à franchir la succession de cataractes qui, sur 250 kilomètres, sépare le Pool de Boma, où l'expédition, décimée et à la fin de ses ressources, arriva enfin, le 9 avril 1877. Il y avait près de trois ans qu'elle avait quitté Zanzibar et neuf mois qu'elle était partie de Nyangwe. Des 356 compagnons nègres que Stanley avait à son départ, 115 seulement arrivèrent avec lui à l'autre côte du continent; ses trois compagnons anglais, Barker et les deux frères Pocock, avaient successivement péri à ses côtés: mais le but du grand voyage était atteint: une vaste courbe était décrite au cœur de l'Afrique équatoriale, l'un des plus grands problèmes de la géographie contemporaine était résolu (1). 》

Plus tard, Stanley accomplira encore deux campagnes dont nous reparlerons plus loin. La deuxième expédition de

(1) A.-J. Wauters. L'Etat Indépendant du Congo, 1899, pp. 16, 17 et 18 . 
Stanley fut une révélation. Elle devait précipiter la reconnaissance et le partage politique des régions traversées. Si, dès lors, les traits généraux de la configuration intérieure de l'Afrique étaient reconnus, partout restaient les explorations locales à accomplir. "Mais il était déjà facile de voir que les explorations allaient perdre de leur caractère désintéressé, et tendaient à servir, outre la science, des intérêts de conquête et de colonisation." (Vidal de la Blache).

Ces explorations locales se poursuivirent sans interruption dans toutes les parties de l'A frique pendant le dernier quart du XIX siècle.

Au moment où Stanley, en plein cœur de l'Afrique, allait entreprendre la véritable grande lutte à travers la forêt du Congo, à Bruxelles, en septembre 1876 , sous l'initiative du roi des Belges Léopold II, se fondait l'Association internationale africaine, dont le but initial était la reconnaissance du centre africain d'après un plan élaboré d'un commun accord. Cette association se décomposait en un certain nombre de comités nationaux qui organisèrent des expéditions. Il faut dire que dans l'esprit de chacun de ces comités existait une arrière pensée politique qui apparut bien dans l'avenir. Le comité belge organisa cinq expéditions (I) dont les résultats ne répondirent pas à l'effort : de I877 à I 885 , entre Zanzibar et le Tanganika, se succédèrent Cambier, Popelin, Carter et Cadenhead, Ramaeckers et Becker, Storms. Quelques stations, Tabora, Karéma et Pala furent bien créées, mais le sacrifice fut grand et presqu'inutile. Des unités de grande valeur périrent sans avoir donné ce qu'on était en droit d'attendre d'elles. Tel fut le sort de Julos Ramaeckers, enlevé en pleine sève et auquel l'histoire n'a pu donner la place qui lui revient dans la reconnaissance du continent

(1) Une sixième expédition organisée, mais non effectuée et arrêtée à Zanzibar n'entre pas ici en ligne de compte. 
africain. Il a en effet en 1877-78 accompli une belle et fructueuse trarersée du Sahara de Tripoli au Tchad. Reparti en Afrique, il mourut à Karéma en I $88 \mathrm{I}$. Les manuscrits et documents de son expédition saharienne ne purent jamais être publiés. Jules Ramaeckers fut oublié.

Les expéditions belges par l'est ne réussirent donc pas. Celles du comité allemand furent plus heureuses et posèrent les premiers jalons de la colonie allemande de l'Est-Africain. Parmi les expéditions du comité français, celle du valeureux Pierre Savorgnan de Brazza jetait les bases du Congo français.

Cependant que la première expédition belge, dirigée par Cambzer, ouvrait la douloureuse série, Stanley victorieux apparaissait au monde émerveillé dans l'estuaire congolien. Sans perdre un instant, dans le plus grand secret et sans abandonner l'organisation des expéditions par Zanzibar, le comité belge élabora un plan nouveau d'attaque du bassin congolien par la voie de l'estuaire et du Stanley Pool. Une société Comité d'études du haut-Congo fut constituée, les principaux membres en étant les premières personnalités $\mathrm{du}$ Comité belge de l'Association internationale africaine. Dès lors, sous l'impulsion donnée par cette société, le bassin du Congo fut fouillé avec une activité sans égale. Beaucoup de Belges prirent part à cette conquête du réseau du grand fleuve, sous la direction de Stanley, revenu en 1878 et reparti en 1879, avec une mission politique nettement définie. M. A. J. Wauters, directeur du Mouvement géographique, qui fut, comme il le dit lui-même, intimement et activement mêlé aux événeménts de ce début, a excellemment résumé les péripéties de la lutte, dans son livre que nous avons déjà cité. Nous y renvoyons le lecteur désireux de se mieux renseigner. Stanley, avec son matériel de navigation fluviale, arrive au Stanley-Pool dont la rive droite était déjà occupée par de Brazza et lance toute une flottille de petits steamers sur le 
fleuve et ses affluents jusqu'à la zone des chutes limitant la haute plaine congolienne.

D'autre part, les confins du bassin étaient reconnus par des explorateurs étrangers partis de l'Afrique orientale.

Pour les Belges, le moment menaçait de devenir critique en raison des revendications qui s'annonçaient. Le Comité d'études du haut-Congo régularisa par des accords et des traités, la situation du nouvel empire colonial qui se trouvait créé. Il devịnt lui-même l'Association internationale $d u$ Congo et poursuivit sans répit la tâche commencée. Les expéditions se succédaient, confiées aux plus ardents, aux plus audacieux, aux plus habiles, belges ou étrangers; les territoires nouveaux se découvrent, des traités innombrables sont passés avec les chefs indigènes; la Congolie presque tout entière constitue le domaine conquis pour l'Association internationale $d u$ Congo dont l'âme et la cheville étaient Léopold II. "Ainsi (en I884), cinq années avaient suffi pour faire, jusqu'au certre du continent, les plus brillantes reconnaissances, visiter pacifiquement cent peuples nouveaux, obtenir des chefs indigènes plus de cinq cents traités de suzeraineté, fonder quarante établissements, jeter sur le haut fleuve, par delà les cataractes, cinq steamers, occuper le pays depuis le littoral jusqu'aux Stanley-Falls, depuis Bangala jusqu'à Luluabourg! L'Europe diplomatique ne pouvait pas rester spestatrice indifférente d'une entreprise aussi audacieuse et déjà couronnée d'autant de succès. ) (I).

Belges, Français, Portugais, Allemands, Anglais parcouraient l'A frique, tous avec l'intention de conquérir les territoires les plus vastes. Le temps était venu : $I^{\circ}$ de partager l'Afrique centrale à l'amiable entre ses conquérants ; $2^{\circ}$ de s'entendre sur le régime commercial à établir dans ces pays, nouvellement ouverts à l'activité européenne.

Cela se fit en 1884 et 1885 .

(1) A.-J. Wauters. Ouvrage cité. 
Malgré les prétentions portugaises et un accord conclu entre le Portugal et l'Angleterre, grâce par contre à un accord avec la France et surtout à l'appui de Bismarck, l'Angleterre

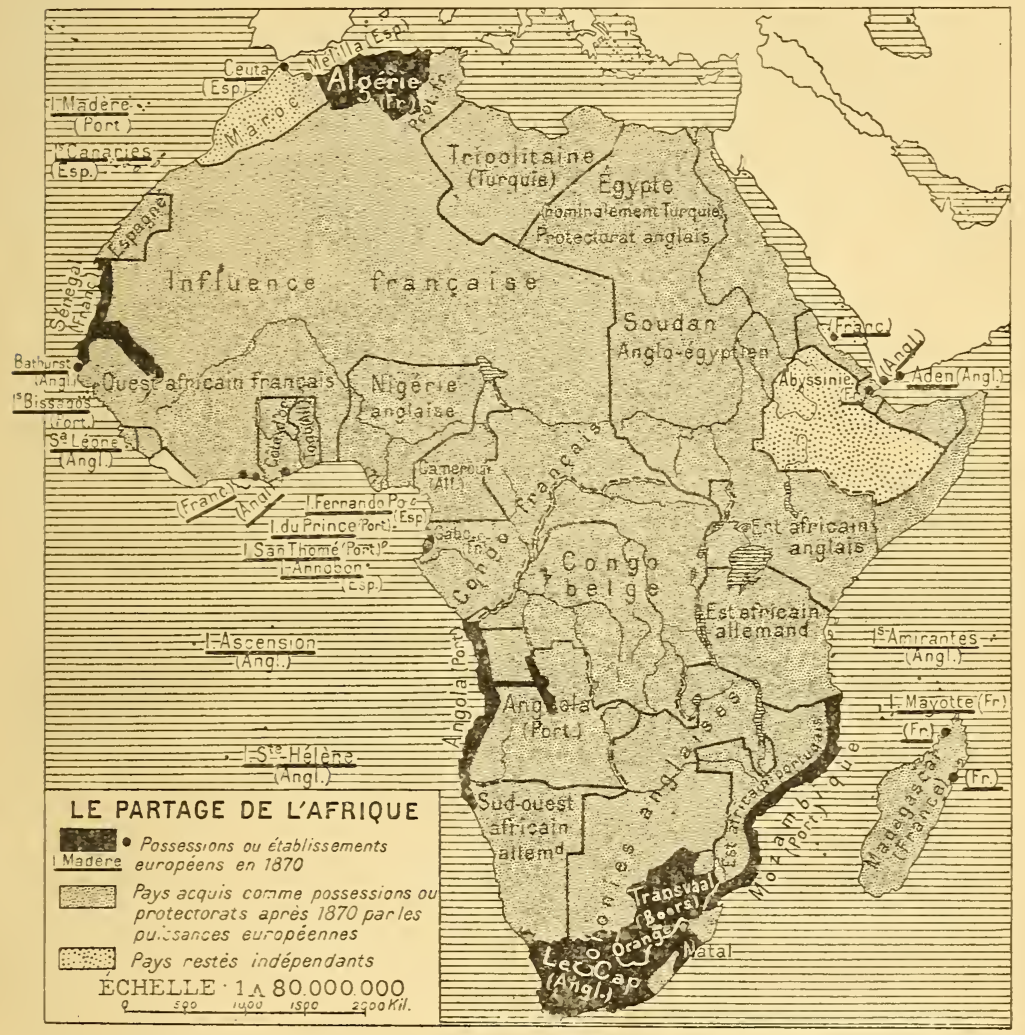

Par erreur de photogravure la petite république de Libéria n’est pas indiquée comme indépendante (petit espace en blanc, côte de Guinée).

Fig. !

se rallia aux vues de Léopold II et dénonça son traité reconnaissant la souveraineté portugaise sur le Bas-Congo.

Sur la proposition de Bismarck, une conférence internationale se réunit à Berlin et de novembre 1884 à février 1885 élabora un traité dit Acte général de Berlin. 
Cet acte stipulait pour tout le bassin du Congo: $\mathrm{I}^{\circ}$ la liberté commerciale absolue, la liberté de conscience, la liberté d'établissement, la protection de la population indigène;

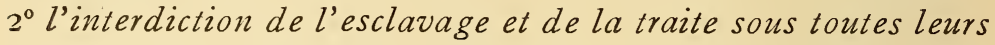
formes ; $3^{\circ}$ la médiation et l'arbitrage obligatoires en cas de conflit surgissant dans les limites du bassin du Congo ou à son sujet; $4^{\circ}$ la liberté de navigation sur le Congo et ses affluents (la route, le chemin de fer ou le canal tenant lieu d'une section non navigable du fleuve est assimilée au fleuve même).

Cependant l'Association internationale du Congo était reconnue Etat souverain successivement par toutes les puissances. Le 23 février I885, l'Etat indépendant du Congo adhérait à l'Acte général de Berlin, trois jours avant la dissolution de la Conférence internationale. Deux mois après, les 28 et 30 avril, la Belgique, par l'intermédiaire du pouvoir législatif, autorisait son roi à devenir le souverain du nouvel État africain.

Aux termes de cet accord, l'union entre la Belgique et cet État devait être exclusivement personnelle, c'est-à-dire que Léopold II, roi des Belges d'une part, était en même temps L.éopold, souverain de l'État indépendant du Congo d'autre part, sans que ces deux États eussent d'autres liens, de quelque nature que ce soit. Le Congo était bien une colonie, il est vrai, mais une colonie personnelle et non nationale. La métropole se synthétisait en un homme : le souverain Léopold C'est par extension ou par erreur que l'on disait le Congo belge. De fondation et de régime, le Congo était l'œuvre de son souverain. Il eût fallu dire le Congo léopoldien.

Après I885, l'exploration des territoires de l'État du Congo se poursuivit sans relâche, particulièrement pendant les dix premières années. George Grenfell, missionnaire protestant anglais, qui avait commencé en I884, mena à bien un grand nombre de reconnaissances sur le Congo et la plupart 


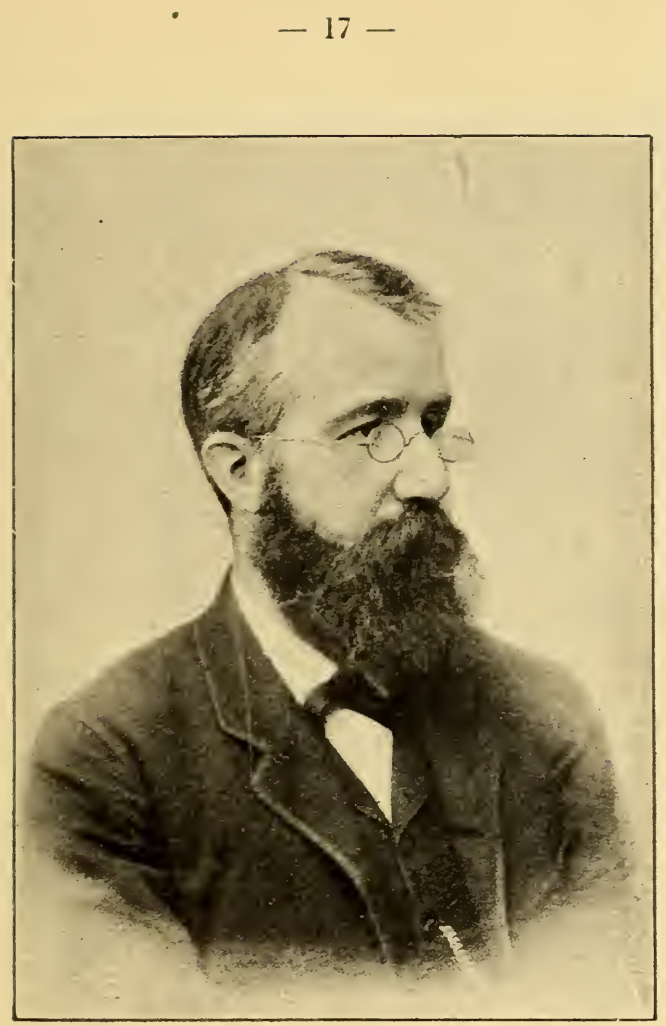

Fig. 5. - George Grenfell, mort le rer juillet 1906 à Basoko, âgé de 57 ans.

« Grenfell explora et évangélisa l'Afrique centrale a la façon du bon docteur Livingstone, dont il avait la petite taille, le calme bienveillant, la douceur native, l'esprit curieux et ouvert. A un grand nombre de peuplades du haut Congo, il a fait connaitre le Blanc, qui, avant lui, n'avait pas encore pénétré parmi elles. 11 y arrivait en homme de pais, gagnait la confiance des indigènes barbares par sa patience, son tact et son habileté, et se gardait bien de répondre par la violence à la brutale défiance de ces étres primitifs. En même temps qu'il ouvrait la route aux agents politiques de l'État et aux représentan's du commerce libre, il éveillait la curiosité des natifs en faveur des Européens et facilitait ainsi la tâche à ceux-ci. Cette mission, pleine de dangers, il l'a poursuivie vingt-cinq ans durant, à l'avant-garde, avec autant d'humanité que de succès. Aussi son nom doit-il être honoré.

- Lorsqu'on songe que la conquête des terres nouvelles est si souvent accompagnée, quoi qu'on fasse, d'abus, d'excès, de manœuvres coupables et d'agissements c ue la civilisation réprouve, il est réconfurtant d'avoir á évoquer le souvenir de cet homme de bien, missionnaire dans la plus pure acception du mot, qui parvint à sillonner de ses itinéraires, à jalonner de ses positions astronomiques, l'immense bassin central du Congo, le rameau d'olivier à la main. "(A.-J. Wauters, Mouvement Géographique, 1o mars 1907.)

Cliché mis gracieusement à notre disposition par le Mouvement géographique. 
des affluents signalés par Stanley, notamment l'Oubanghi dont il est le découvreur incontesté. Les Portugais Capello et Ivens explorèrent le Katanga. Wissmann, allemand (I884I887), apporta la grosse découverte du Kasaï et de ses principaux tributaires. Stanley réapparaît en Afrique. Le I8 mars, à Banana, il est à la tête de l'expédition anglaise dont le but est la délivrance d'Emin Pacha, bloqué sur le haut Nil par les Mahdistes. Deux ans sont employés à la réalisation de ce but ( 7 février I889). Les nouvelles découvertes géographiques de Stanley sont considérables: il remonte l'Arouwimi, arrive sur le Nil, reconnaît la rivière Semliki, le puissant massif du Rouvenzori et le lac Albert-Édouard.

Parmi les Belges qui prirent une part extrêmement active aux explorations du bassin congolien, nous citerons, pour être bref les noms les plus marquants : tout d'abord Alexandre Delcommune, qui fit de nombreuses expéditions, dont une, particulièrement fructueuse, de I890 à I894. C'est Delcommune qui découvrit en vérité le Katanga en un itinéraire long et difficultueux (Congo, Lomami, jusqu'à son point extrême de navigation - Bena-Kamba, Loualaba, lac Kisalé, Mitoumba, Katanga supérieur, Nzilo, Tanganika, Loukouga, Lousambo, Sankourou). Après Delcommune, par ordre de dates: Van Gele qui, en I888, reconnut définitivement l'Oubanghi-Ouellé; Delporte et Gillis (I890-I89I), dont les travaux d'ordre uniquement scientifique constituèrent la base première de la cartographie du bassin, Van Kerckhoven (189I), Paul Le Marinel (I890-9I) au Katanga, Cornet (189I-92) en reconnaissance scientifique extrêmement fructueuse au Katanga, Brasseur (1896) dans l'Ouroua, Lemaire (I899-1900) dont l'expédition exclusivement scientifique du Katanga, plus encore que celle du Congo-Nil (I902-03-04), reste un modèle, Cabra (I90I-02-03), dont les travaux topographiques fixent les limites entre l'État et les possessions portugaises des deux côtés du bas-fleuve. Une quantité de 
noms belges et étrangers seraient encore à citer dans un travail d'un cadre moins restreint que celui-ci. Les travaux de la Mission mixte anglo-congolaise du $30^{\circ}$ méridien qui viennent d'être terminés seront prochainement publiés. Enfin des recon-

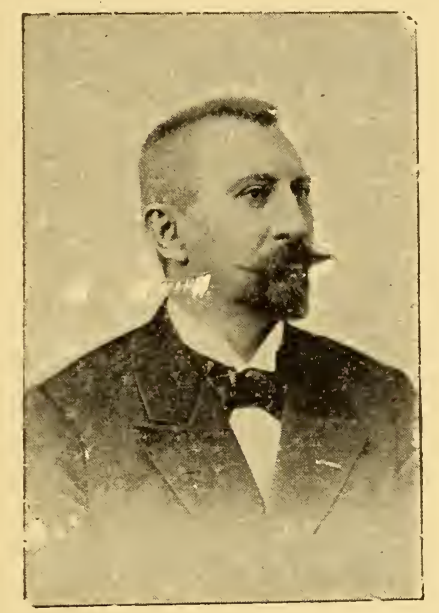

Fig. 6. - ALEXANDRE DELCOMMUNE

Cliché mis gracieusement à notre disposition par le Mcuz'ement géographiqué.

naissances topographiques locales s'opèrent constamment en différents points. Hâtons-nous de dire que la connaissance de ces 2,350,000 kilomètres carrés en est encore tout au début et que toutes les cartes qui jusqu'ici ont été publiées ne peur'ent être considérées que comme des esquisses générales. I.a cartographie scientifique dic Congo sera-t-elle établie dans cent ans? Avec beaucoup de travail et de système, peut-être.

Depuis son érection en État indépendant, la colonie léopoldienne s'est grandement développée et a pris un essor commercial considérable. La Belgique n'a pas été étrangère à ce progrès: $I^{\circ}$ par l'aide pécuniaire qu'elle apporta au jeune État et $2^{\circ}$ par la collaboration individuelle spontanée d'un grand nombre de ses citoyens. 
La Belgique autorisa, en 1887, l'émission par l'État du Congo, d'un emprunt à primes de 150 millions de francs. En juillet I889, elle prêta son concours financier en vue de la construction du chemin de fer des chutes Livingstone. En août I889, le roi léguait par testament le Congo à la Belgique.

La colonie se développait, elle avait besoin d'aide financière. C'est à cette occasion que le testament du Roi fut rendu public en. juillet I 89o. "... J'ai cru — disait le Roi dans une lettre accompagnant la copie de son testament qu'il adressait en 1889 au chef du Cabinet, M. Beernaert - de mon devoir de mettre la Belgique à même, lorsque la mort viendra me frapper; de profiter de mon œuvre ainsi que du travail de ceux qui m'ont aidé à la fonder et à la diriger et que je remercie une fois de plus. J'ai donc fait, comme Souverain de l'État indépendant du Congo, le testament que je vous adresse ; je vous demanderai de le communiquer aux Chambres législatives au moment qui vous paraîtra le plus opportun. "Plus loin le Roi ajoutait : " Laissez-moi, en attendant, vous dire combien je suis reconnaissant, envers les Chambres comme envers le Gouvernement, pour l'aide qu'ils m'ont prêtée à diverses reprises dans cette exécution. "

La Belgique accueillit favorablement la demande d'aide du Congo formulée un an après le dépôt du testament royal. Une convention fut conclue disant en substance :

$I^{\circ}$ L'État belge prêtera à l'État indépendant du Congo une somme de 25 millions de francs. Mode de versement: 5 millions aussitôt après l'approbation par la Législature et 2 millions par an pendant dix ans. Pendant ces dix années, les sommes prêtées ne produiront pas d'intérêt.

$2^{\circ}$ Six mois après l'expiration de ce terme de dix ans, l'État belge pourra s'annexer l'Etat indépendant du Congo.

$3^{\circ}$ Dès à présent, l'Êtat belge recevra tels renseignements qu'il jugera désirables sur la situation économique, commer- 
ciale et financière de celui-ci. Ces renseignements ne doivent avoir pour but que d'éclairer le gouvernement belge et celui-ci ne s'immiscera en aucune manière dans l'administration de l'État indépendant du Congo, qui continuera à n'être rattaché à la Belgique que par l'union personnelle des deux couronnes. Toutefois, l'État du Congo s'engage à ne contracter désormais aucun nouvel emprunt, sans l'assentiment du gouvernement belge.

$4^{\circ} \mathrm{Au}$ terme prédit, la Belgique n'annexant pas le Congo, la somme prêtée ne serait exigible qu'après un nouveau terme de dix ans, mais serait pendant ce noureau terme productive d'un intérêt de 3 i $/ 2$ p. c.

Entre l'État belge et l'État indépendant du Congo, il y avait désormais non un lien politique ou administratif, mais autre chose néanmoins qu'un lien personnel. La Belgique avait acquis des droits. Cependant la mise en valeur et en exploitation du Congo absorbe toutes les ressources et le Gouvernement de l'État indépendant, en I892, contracte un un emprunt secret auprès d'un banquier anversois, M. De Browne de Tiège. Cet emprunt, au taux d'intérêt annuel de 6 p. c., remboursable le $\mathrm{I}^{\text {er }}$ juillet $\mathrm{I} 895$ par 5,287,4 6 francs, est consenti sur véritable hypothèque à courte échéance d'environ Іб millions d'hectares de territoire congolien.

Le ${ }^{\text {er }}$ juillet 1895 approchant, les finances de l'État indépendant ne lui permettaient pas encore de liquider sa dette envers M. De Browne de Tiège. Il s'adressa au gouvernement belge au début de 1895 et proposa l'annexion pure et simple. procédé qui eût affermi définitivement la situation politique et financière de la colonie. La discussion fut longue et mouvementée tant au Parlement que dans la presse et le public. L'annexion fut ajournée. En juin, la Belgique consentit à rembourser la dette De Browne de Tiège et à faire à l'État indépendant un prêt de 1,517,000 francs pour établir la balance de son budget. Le projet d'annexion déposé en janvier, fut retiré en novembre. 
Telle fut la genèse des droits de la Belgique sur la colonie congolienne. Permettons-nous d'abréger. C'est au cours de I 908 que l'annexion se fit. Le 20 août, la Chambre des représentants vota la reprise. Le 9 septembre, le Sénat ratifia la décision de la première chambre. Le i 8 octobre, un arrêté royal promulguait la loi d'annexion et le 15 novembre 1908 , la Belgique assumait l'exercice de son droit de souveraineté sur le Congo. Depuis, il n'y a plus d'État indépendant du

\section{IAA DÉCOUVERTE DE L'AfRIQUE}

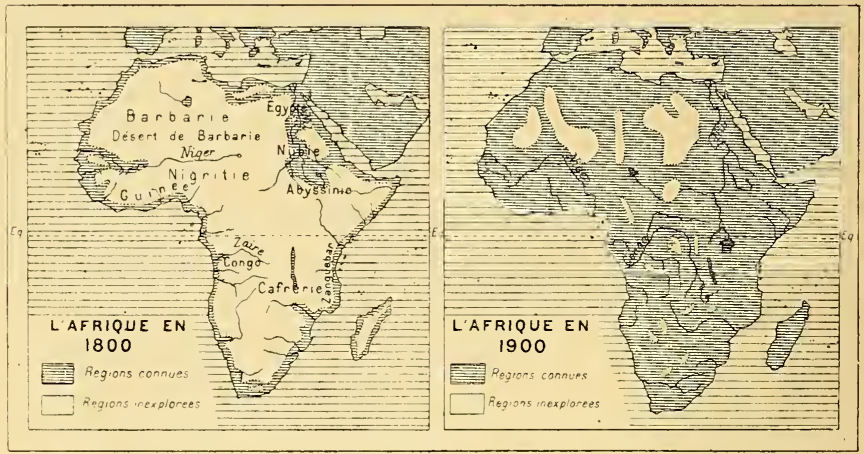

Fig. 7

Congo, il y a la Colonie nationale belge du Congo. La Belgique se trouve au nombre des grandes puissances coloniales.

Le XIX ${ }^{\ominus}$ siècle découvrit l'Afrique et amena son partage politique. La colonisation européenne s'y engagea et en poursuivit l'exploitation sans songer même à en tenter le peuplement par des colons européens, si ce n'est dans les régions du nord et du sud affectées de climat tempéré chaud. Actuellement restent seuls indépendants en Afrique, l'Empire du Maroc, dont la situation intérieure et extérieure est extrêmement précaire, l'Empire quasi féodal d'Abyssinie, qui a affirmé sa forte vitalité il y a quelques années dans sa lutte contre 
l'Italie (1896) et enfin la petite République nègre de Libéria, fondée en 1822 par des nègres américains, qui ne doit son indépendance qu'à une protection toute morale des EtatsUnis de l'Amérique du Nord. Les Républiques Sud-Africaines du Transvaal et d'Orange, colonies de peuplement en pleine activité, perdirent leur indépendance en mai 1902 après trois années d'une héroïque défense contre l'Angleterre. 



\section{LE MILIEU NATUREL}

I. Position ASTRonomique et SITUATION GÉOGRAPHIQUE. - La longitude des territoires du Congo belge correspond à celle de l'Europe centrale en sorte que l'écart entre le temps de Greenwich, qui est le nôtre, officiellement du moins ( $\mathrm{I}$ ), et le temps du Congo ne peut être considérable. Nos horloges marquant I 2 heures, il est à Boma exactement I 2 h. 52 m. 25 s. ( $13^{\circ} 6^{\prime}$ Io" ' Long. Est Greenwich - Delporte \& Gillis) et aux confins orientaux extrêmes du Congo, sous ie $30^{\circ}$ méridien Est de Greenwich, il est I4 heures.

Les possessions belges, entièrement comprises entre les latitudes $5^{\circ} 30^{\prime}$ nord et $\mathrm{I} 3^{\circ} 30^{\prime}$ sud, se trouvent dans la zone équatoriale. Par ce fait, les rayons solaires, peu inclinés sur le plan de l'horizon, dardent lumière et chaleur puissantes. Le jour se voile et s'éteint rapidement en un crépuscule plus court que le nôtre et la durée de la nuit s'écarte peu de la

(1) Pour la facilité des relations internationales, le temps de Greenwich a été adopté par l'administration de nos chemins de fer. Par la force des choses, l'usage général de cette heure s'en est suivi dans tous les rouages de notre vie sociale et pricée. Mais en réalité, lorsque nos horloges officielles marquent les 12 heures temps moyen de Greenwich, le soleil est déjà passé à notre méri. dien depuis $0 \mathrm{~h} .17 \mathrm{~m} .26 \mathrm{~s} .9$ (Uccle Observatoire 4' 21' 44" Long. Est (rreenwich). 
durée du jour (I); en un mot, le régime équinoxial règne au Congo comme en Amazonie en raison de très basses latitudes. Les climats de ces contrées sembleraient devoir correspondre si des différences considérables dans les conditions d'alti-

Étendues comparées de La BeLGique et de son domaine Colonial $/ 1$ A L'ÉCHELLE DE I A 25000.000

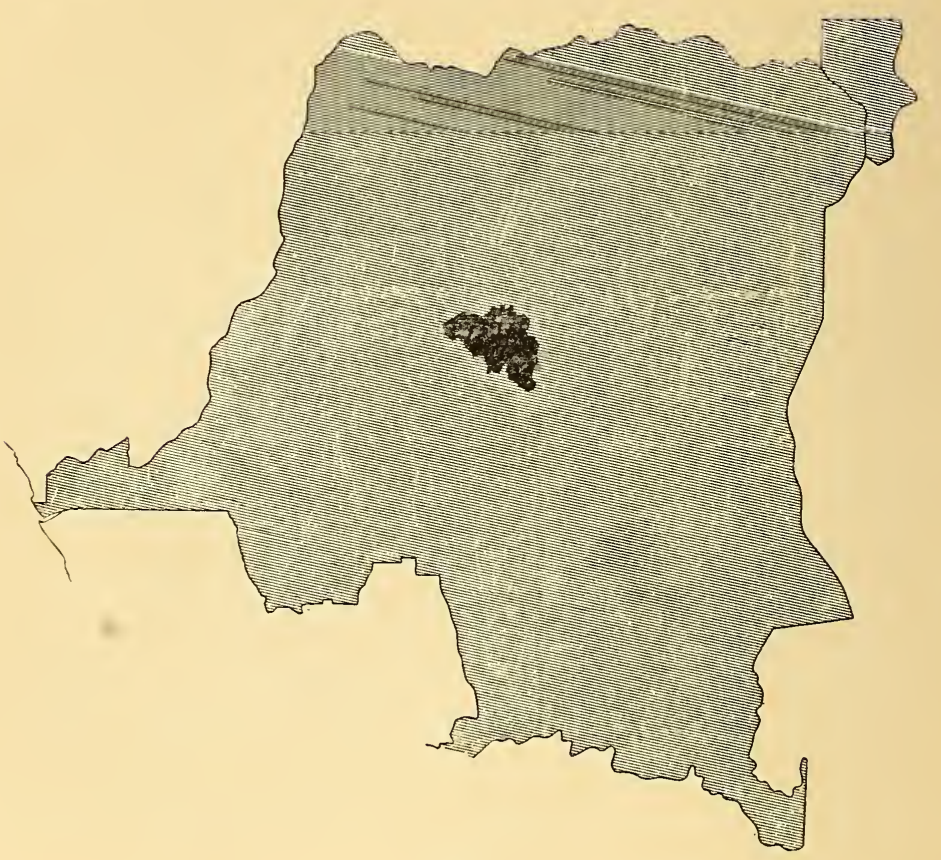

Fig. 8

tude et de disposition continentale ne faisaient dévier les effets climatériques de la position astronomique. L'Amazonie

(1) Nous entendons ici comme durée de la nuit l'intervalle de temps qui s'écoule entre le coucher et le lever du soleil. Ainsi donc la durée de la nuit, y compris les deux crépuscules courts du soir et du matin, diffère peu de la durée du jour plein. 


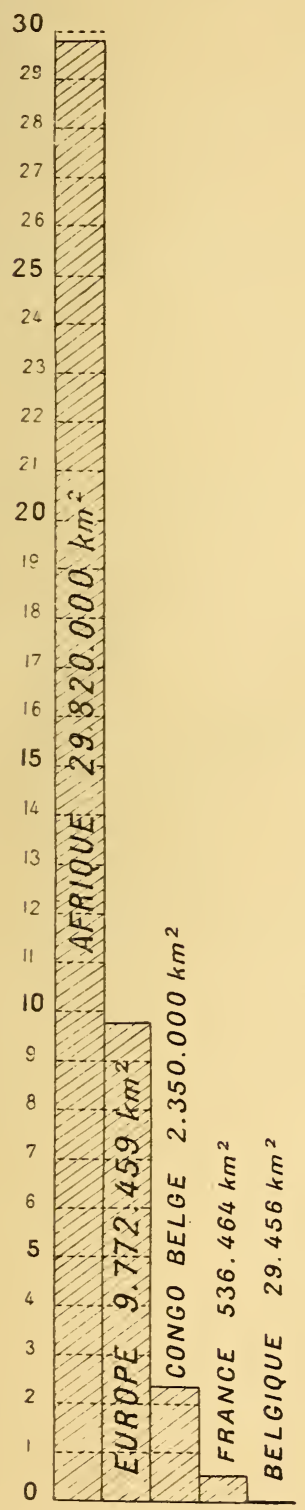

Fig. 9 est une énorme plaine basse largement ouverte aux effluves océaniques. L'Insulinde est plongée dans les flots d'une mer chaude. Le bassin congolien au contraire est une grande plaine continentale déjà élevée - 300 à 500 mètres - totalement fermée du côté de l'est à l'influence de l'Océan des Indes par le haut socle tabulaire des grands lacs et dégagée en grande partie à l'occident de l'action atlantique par la disposition en barrières des alignements montueux de l'ouest.

Par sa situation au milieu d'un continent très massif et élevé, par l'étendue considérable de domaines très rama'ssés - 2,350,000 kilomètres carrés, I/I 3 de l'A frique, 80 fois la Belgique - le bassin congolien constitue un milieu équatorial original, différent de celui de l'Insulinde ou de l'Amazonie.

II. LE Sol. - Le relief. - Toute l'Afrique équatoriale de l'Océan Indien à l'Océan Atlantique, présente bien le type du relief tabulaire africain : à l'est, haut plateau évidé; à l'ouest, vaste plaine concave située à une altitude de 300 à 500 mètres et s'élevant graduellement dans toutes les directions. Ce vaste bassin intérieur, draîné par un des plus riches réseaux hydrographiques du monde, ne présente qu'une ouverture vers la mer, un goulot que se sont creusé et au travers 
lequel se ruent les eaux de toutes les rivières rassemblées en le formidable collecteur central qu'est le fleuve Congo. Dans la zone côtière, ce courant s'assagit et se déverse à l'Océan en un imposant estuaire bordé au nord et au sud par une petite plaine maritime.

Ainsi nous voyons bien la disposition du bassin du Congo et du plateau oriental de l'Afrique allemande, séparés l'un de l'autre par une zone très tourmentée, marquée de crevasses et d'enfoncements du sol suivant l'alignement des grands lacs Tanganika, Kivou, Albert, Albert-Édouard.

Remontant le Congo et ses affluents, nous parcourons une série de terrasses étagées, séparées les unes des autres par des pentes plus raides ou des rebords abrupts, sur lesquels les eaux se précipitent en rapides ou sautent en chutes.

Point de grandes et longues chaînes de montagnes. Les Plateaux montagneux de l'ouest et les Mitoumba, à l'est et au sud-est sont de puissantes surélévations parcourues par des chaînons plus ou moins courts, de direction souvent parallèles. Ces deux régimes montagneux aident à reconnaître trois parties principales dans le bassin congolien :

$\mathrm{I}^{\circ}$ La région atlantique, peu large (maximum 300 kilomètres), plaine côtière sur laquelle s'évase le lit du fleuve engorgé à la traversée du haut relief occidental ;

$2^{\circ}$ La région du milieu, en amont du confluent Congo-Kasaï, encadrée par les plateaux montagneux de l'ouest, le plateau de Lounda, les Mitoumba, la ceinture des grands lacs et son double ourlet montagneux, enfin les hauteurs tabulaires du nord qui s'abaissent en un seuil de moins de 500 mètres d'altitude et mettent en communication aisée les bassins du Tchad et du Congo. Cette région du milieu est elle-même répartie en deux principales terrasses dont le rebord de séparation est franchi en rapides et en chutes par le Congo et tous ses affluents. Ce rebord s'étend du Kwango, au sud-ouest, à l'Ouellé, au nord-est, sur la zone parcourue par les sinuo- 


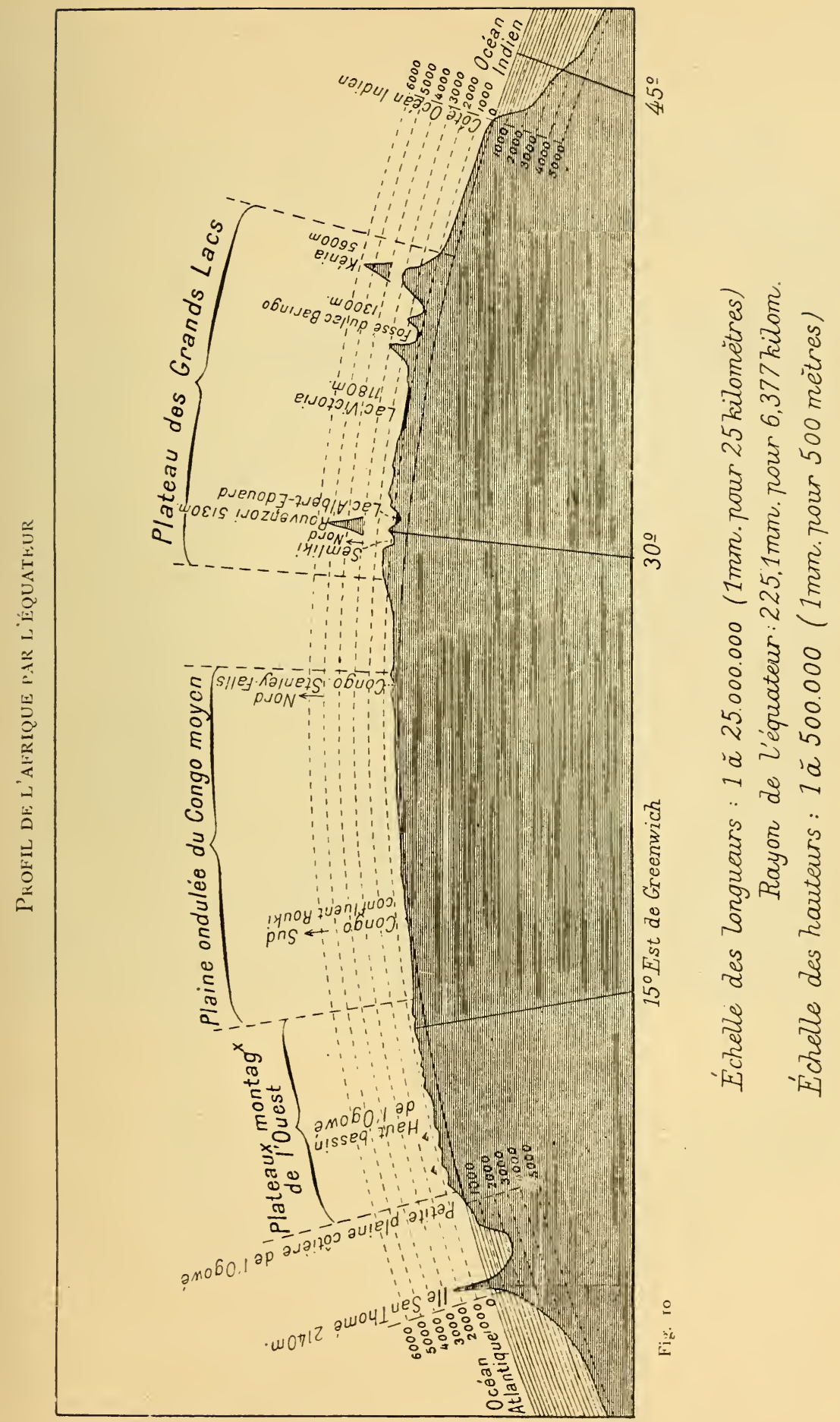


sités de la courbe de niveau de 500 mètres. Ces zones de rapides délimitent nettement les cours supérieurs des rivières congoliennes de leurs cours moyen ou inférieur. De ces deux

\section{LA DISPOSITION DU RELIEF CONGOLIEN}

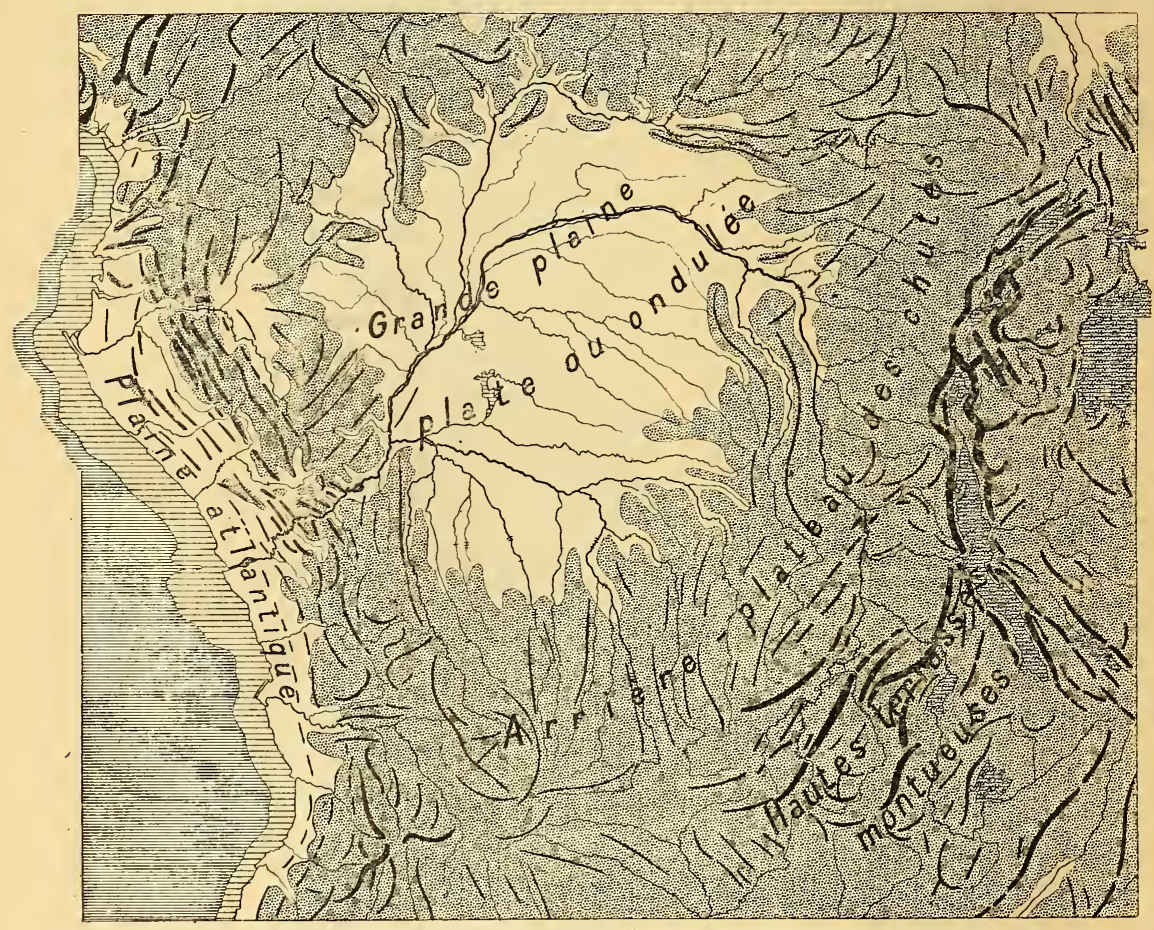

Fig. II

Échelle : 1 à 25:000.000

1000kil

terrasses centrales, l'une est la grande plaine plate ou très peu ondulée, occupée jadis par un grand épanchement lacustre et caractérisé aujourd'hui par le plus grand développement fluvial. Quant à la plus élevée des deux terrasses, elle est affectée d'un relief plus accusé et plus varié, c'est l'arrière 
plateau congolien coupé transversalement par les coulières agitées des innombrables attluents du Congo;

$3^{\circ}$ La haute région du Katanga et du Tanganika, dominant de 500 à 750 mètres l'arrière plateau congolien. Elle-

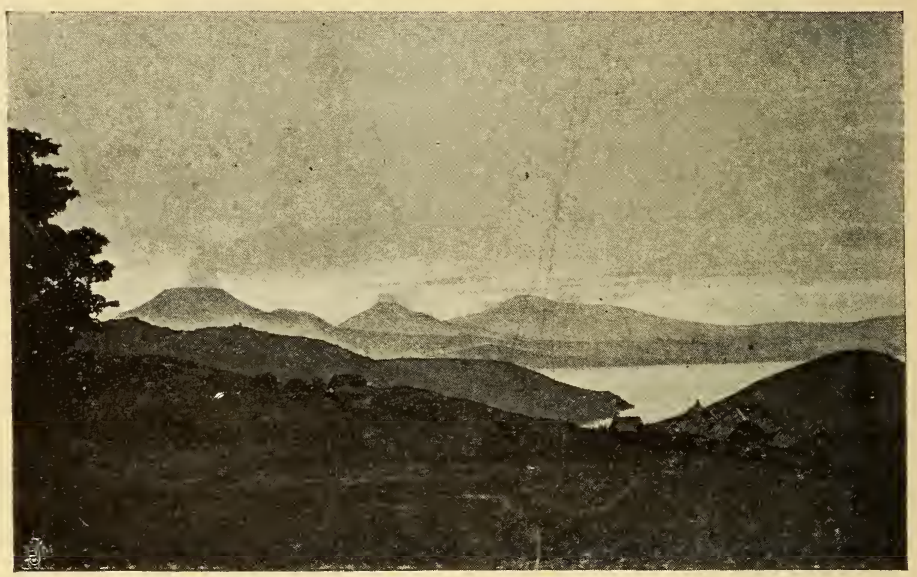

Fig. 15. - LE Pays des volcans aU NORD dU LAC K1vou. - Huit priucipaux cônes volcaniques dont six dépassent $3,500 \mathrm{~m}$. (maximum : Karisimbi 4,500 m.) Ce groupe est en activité. En mai 1904, éruption observée du Kirounga Tchaniragongo (3.400 m.) qui, sur cette photographie, émet encore d'abondantes vapeurs.

(Phctographie communiquée par le Ministère des Colonies.)

même est un vaste plateau montueux d'altitude moyenne supérieure à I,500 mètres, balafré de fractures, sillonné de vallées et de graben (I) et se dissociant en un grand nombre de terrasses étagées: du Nzilo, du Moéro, du Bangwélo, du Tanganika, du Kivou. Cette dernière est la plus élevée du bassin entier; elle est limitée au nord par le puissant massif

(1) Fossé allongé provoqué par un affaissement entre deux fractures parallèles. 
volcanique des Virounga qui barre et domine le fossé des grands lacs (I).

Un second groupe volcanique, d'altitude encore supérieure à celle du premier, le Rouvenzori, émerge du fond du graben au nord du lac Albert-Édouard. Plusieurs de ses sommets dépassent 5,000 mètres.

La structure et la nature du sol. - La nature du sol du pays congolien correspond bien à son aspect orographique. Il semble que la grande dépression centrale entourée d'un cadre montagneux et tabulaire existait déjà à l'époque très ancienne dite ère primaire et que peu à peu, pendant toute la durée des temps géologiques, se sont successivement déposés dans cette plaine concave des grès et des schistes rouges, puis des grès blancs friables. Toutes ces couches ont disparu sous un manteau de terres meubles, provenant de l'altération chimique et de la désagrégation mécanique des roches. Tantôt, ces débris sont restés en place, tantôt, ils ont été remaniés par les eaux et déposés ailleurs sous forme d'alluvions.

Ces formations détritiques sont très importantes au Congo, particulièrement sur les plateaux et les plaines. La nature du sous-sol influe sur leur composition : tantôt elles sont argileuses comme dans certaines parties du Katanga à sous-sol schisteux. Tantôt elles sont sableuses comme dans la grande région du milieu dont le sous-sol est de grès blanc friable. Quant aux alluvions, elles sont aussi argileuses ou sablonneuses, suivant qu'elles se sont déposées dans les eaux calmes ou agitées.

La terre congolienne se présente souvent sous une couleur brun-rougeâtre qui frappe tous les voyageurs. Cette couleur provient de l'oxydation énergique des matières ferrugineuses

(1) Voir plus loin sous la rubrique "Les rivières» des photographies se rapportant aussi au relief. 
du sol sous l'influence des copieuses pluies congoliennes chargées de divers principes chimiques. Ces matières sont transformées en limonite qui colore les terres en jaune, roux ou rouge-brique suivant la proportion. Les dépôts détritiques

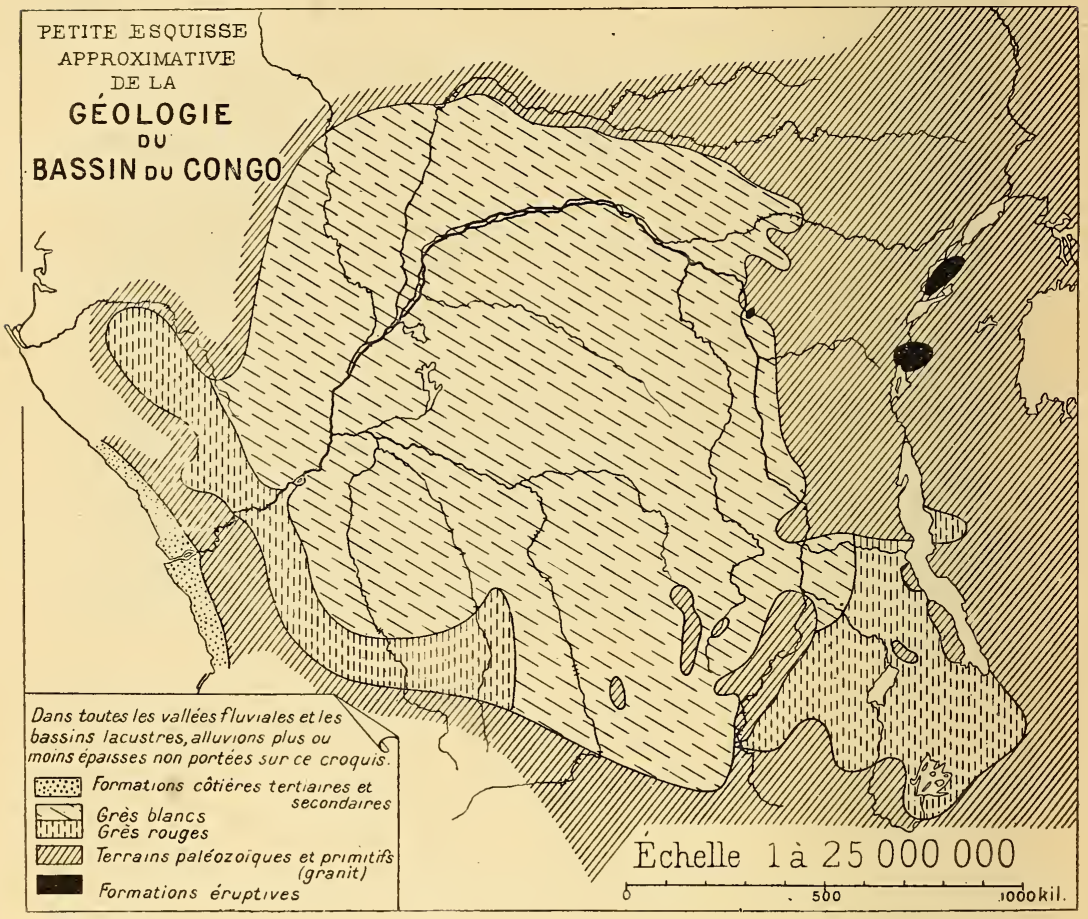

Fig. 15.

(D'apris St t.nicr)

ainsi transformés, portent le nom de latérite (du latin later, brique), terre aride si elle est sèche, d'une grande fertilité si elle est suffisanment arrosée.

III. - LE CLIMAT. - Chez nous, c'est la température qui marque les saisons. Au Congo, la température est assez. uniforme, par contre les pluies sont périodiques et c'est l'allure de leur chute qui détermine la marche des saisons. 
La température.-Pour la plaine, la température augmente à mesure que l'on avance vers l'intérieur (Banana, moyenne annuelle $26^{\circ}$; au nord de la colonie : $30^{\circ}$; dans le Katanga, la grande altitude fait tomber cette noyenne jusqu'à $23^{\circ}$ ). La position en latitudes nord et sud des territoires du Congo entraîne l'alternance des périodes de plus fortes et de moindres chaleurs pour les régions situées de part et d'autre de l'Équateur. Au sud du deuxième parallèle sud, juillet est en général le mois le moins chaud, février le mois le plus chaud (à Banana mars est le mois le plus chaud). La saison chaude dure de novembre à avril, la saison la moins chaude de juin à septembre. Au nord du deuxième parallèle nord, l'inverse se produit. Dans la zone équatoriale intermédiaire, règne un régime de transition à variations très faibles.

Faible variation annuelle du thermomètre est le fait duminant de la climatologie des pays congos. Par contre, le balancement diurne est plus grand qu'en Belgique: $8^{\circ} 5$ Banana; cet écart augmente à l'intérieur: $13^{\circ}$ à Loulouabourg.

"Cette question de la variation diurne de la température au Congo a une grande importance au point de vue de l'hygiène: elle est souvent la cause de beaucoup de maladies, tant chez les blancs que chez les noirs. Lorsqu'après une journée très chaude se produit, la nuit, un abaissement thermique prononcé, il peut en résulter de graves conséquences pour ceux qui ont négligé de prendre les précautions indispensables. „ (Lancaster).

En général, les plus hautes températures du jour se produisent en mars, elles atteignent jusqu'à $33^{\circ}$. I_es plus basses températures surviennent en juillet, elles descendent en général jusqu'à $\mathrm{I} 8^{\circ}$ dans les altitudes considérables. Au Katanga, l'expédition Lemaire a relevé $2^{\circ}$ au-dessus de 0 .

« Au Congo inférieur, la saison comprise entre le milieu de juin et le commencement de septembre est sans contredit la plus agréable, la plus belle et aussi la plus saine de l'année. La température est modérée, le soleil n'incommode pas et les nombreux après-midi sans nuages stimulent l'esprit; les rares journées couvertes, pendant lesquelles le soleil n'est pas visible un seul 
instant, rompent la monotonie et permettent de faire des excursions ou des parties de chasse. Le voile bleuâtre de brouillard étendu sur le paysage, les herbes jaunies, les nombreux arbres

Diagramme Comparatif DE LA MARChE ANNUELle DE LA TEMPÉRATURE

AU CONGO (Moyenre de 15 Stations.)

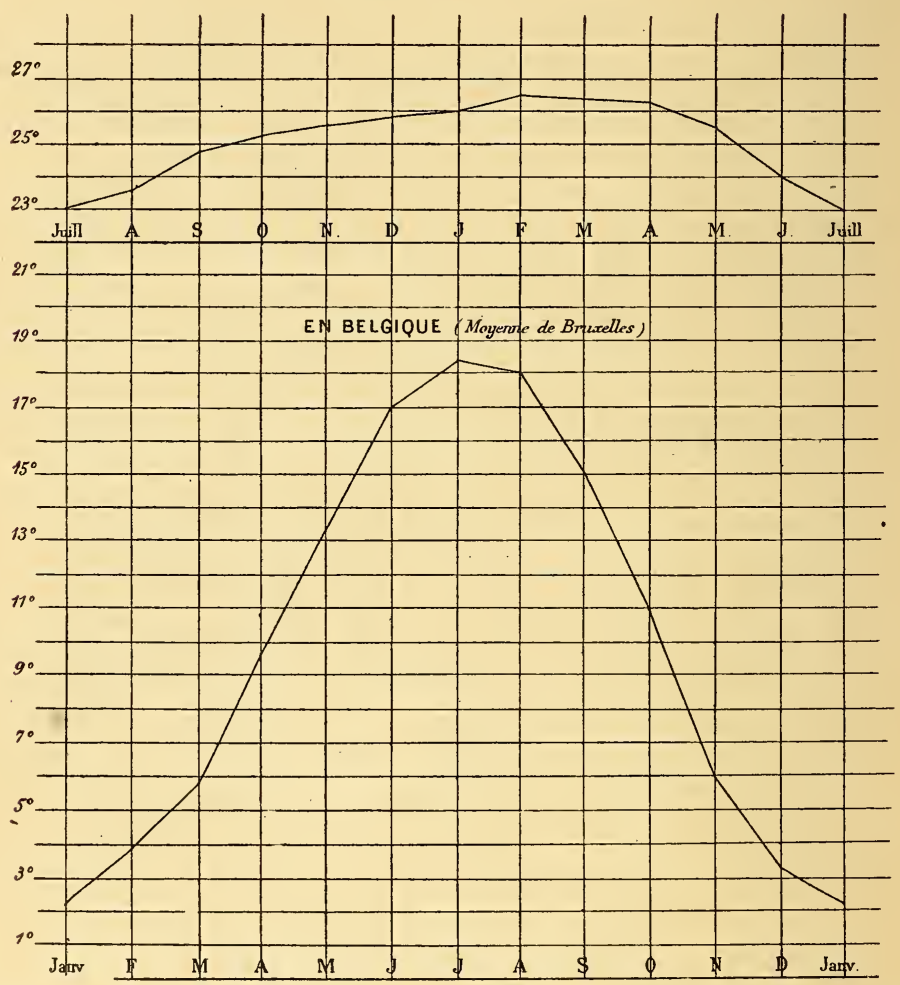

Fig. 17 .

(Lancaster et Meuleman, Le climat du Congo).

dépouillés, le silence de la nature, que vient seul interrompre le roucoulement lointain du pigeon gris qui niche dans les bouquets d'arbres répandus sur les montagnes, tout offre un charme particulier et vient rappeler les belles journées d'automne de l'Europe centrale. 
" La chaleur est parfois, pas toujours, accablante dans le cours de la saison des pluies, surtout en février et pendant la première quinzaine de mars, car les orages sont rares en cette période et l'atmosphère n'est presque jamais rafraîchie par la pluie qui les accompagne. Mais à d'autres époques encore de la même saison,

\section{VARIATION DIURNE DE LA TEMPÉRATURE}

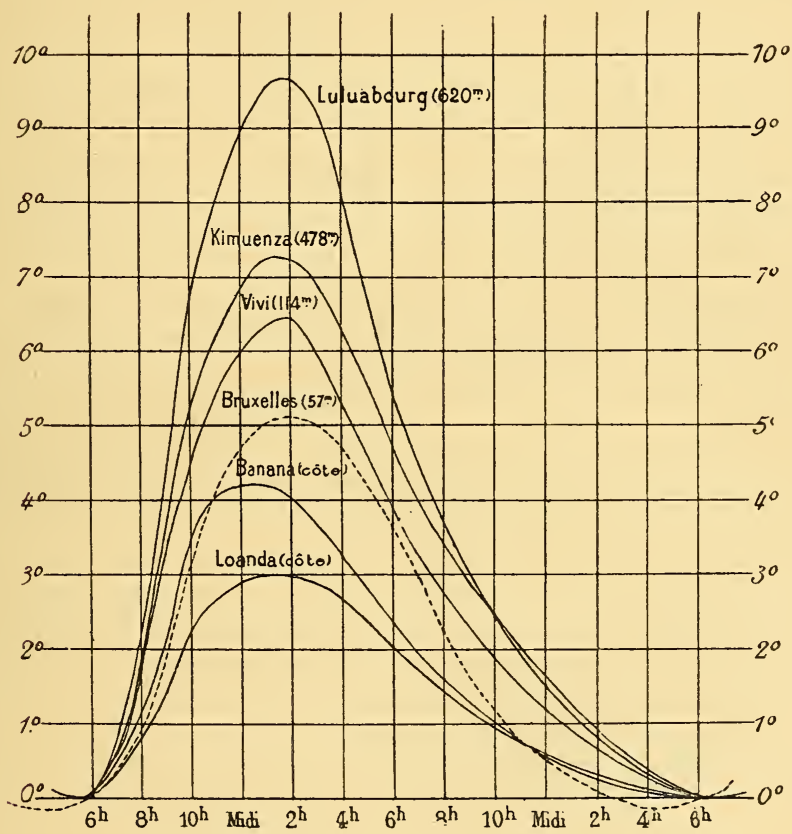

Fig. 18

(Lancaster et Meuleman, Le climat du Congo).

lorsque le soleil darde ses rayons brûlants sur le sol mouillé, la chaleur humide peut devenir étouffante "(von Danckelmann).

a Si la grande variabilité du climat dans nos pays de l'Europe accidentale offre des dangers, en hiver surtout, au point de vue de la santé publique, elle est d'autre part un stimulant à l'activité des fonctions de l'organisme. Dans la zone équatoriale, comme 
nous venons de le voir, l'état thermique moyen est, le plus souvent, au cours de l'année, voisin de $30^{\circ}$ l'après-midi et de $20^{\circ}$ la nuit, avec de très faibles changements d'un jour à l'autre. Si cette situation maintient le corps dans un équilibre de température plus régulier, plus uniforme que chez nous, elle exerce d'autre part sur l'Européen une action débilitante et amollissante que l'on ne peut combattre et écarter qu'en se soumettant aux exigences du régime que commandent pareilles conditions climatériques ») (Lancaster in op. cit. A. J. Wauters).

Les saisons. - C'est la pluie qui caractérise les saisons au Congo, particulièrement dans la partie occidentale, car au centre et surtout dans la région équatoriale, il pleut de façon plus ou moins régulière à tous les moments de l'année. $\mathrm{Au}$ sud $\mathrm{du} 2^{\circ}$ parallèle sud d'octobre à mai règne la saison des pluies, de juin à septembre règne la saison sèche.

Les pluies du Congo ne sont pas diluviennes. Les fortes averses sont comparables à nos pluies d'orages d'été. De peu de durée, elles sont intenses et accompagnées presque toujours de phénomènes électriques et quelquefois de fortes rafales de vent (tornades).

“ En saison sèche point de pluie, rarement une légère brume de 5 à 10 minutes de durée. En revanche, un temps grisâtre, une brise qui fait sur les colons l'effet de nos froides bises d'hiver. La végétation, exubérante de vie et de force, lutte longtemps, cherchant à se soutenir malgré la privation d'eau; mais la sécheresse finit par l'emporter. Tout s'étiole et se fane et lorsque le temps sec a accompli son œuvre, de tous les points de l'horizon s'élèvent des colonnes de fumée annoncant le commencement des incendies annuels des herbes... Les plaines dénudées, portant encore la trace $\mathrm{du}$ feu, laissent alors une triste impression au spectateur, et cet état perdure jusqu'aux premières pluies, qui, par un effet magique, rendent en peu de temps au pays son aspect riant et sa luxuriante végétation »)(Lancaster).

Vers l'intérieur du pays, la différence entre saisons sèche et pluvieuse est moins tranchée et vers l'équateur il n'y a pour ainsi dire plus de saison sèche. 
Nous donnons ici les diagrammes pluviométriques de cinq positions climatériques typiques : $\mathrm{I}^{\circ}$ Banana à la côte; $2^{\circ}$ Kimouenza à quelques kilomètres au sud-est de Léopoldville, au delà de la barrière des plateaux montueux atlanti-

LA Marche annuelle moyenne des Pluies a Banana

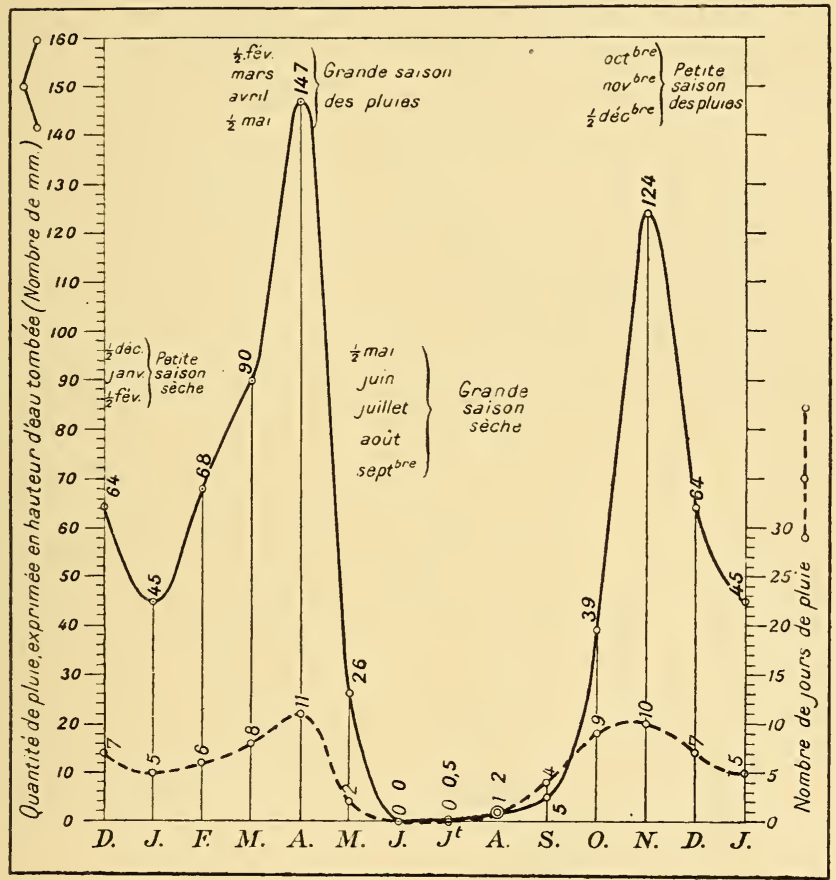

Fig.'I9

(D'après les observations météorologiques des agents de l'Etat.)

ques, à l'issue de la vaste plaine du Congo moyen; $3^{\circ}$ Éala, sur l'équateur; $4^{\circ}$ Mono qui caractérise le régime oubanghien et $5^{\circ}$ Loukafou qui exprime celui du Katanga. La lecture de ces diagrammes, très suggestive, permettra un peu plus loin de se rendre assez bien compte du régime hydrographique $d u$ bassin.

Quant à la quantité d'eau qui tombe sur la durée d'une 


$$
-40-
$$
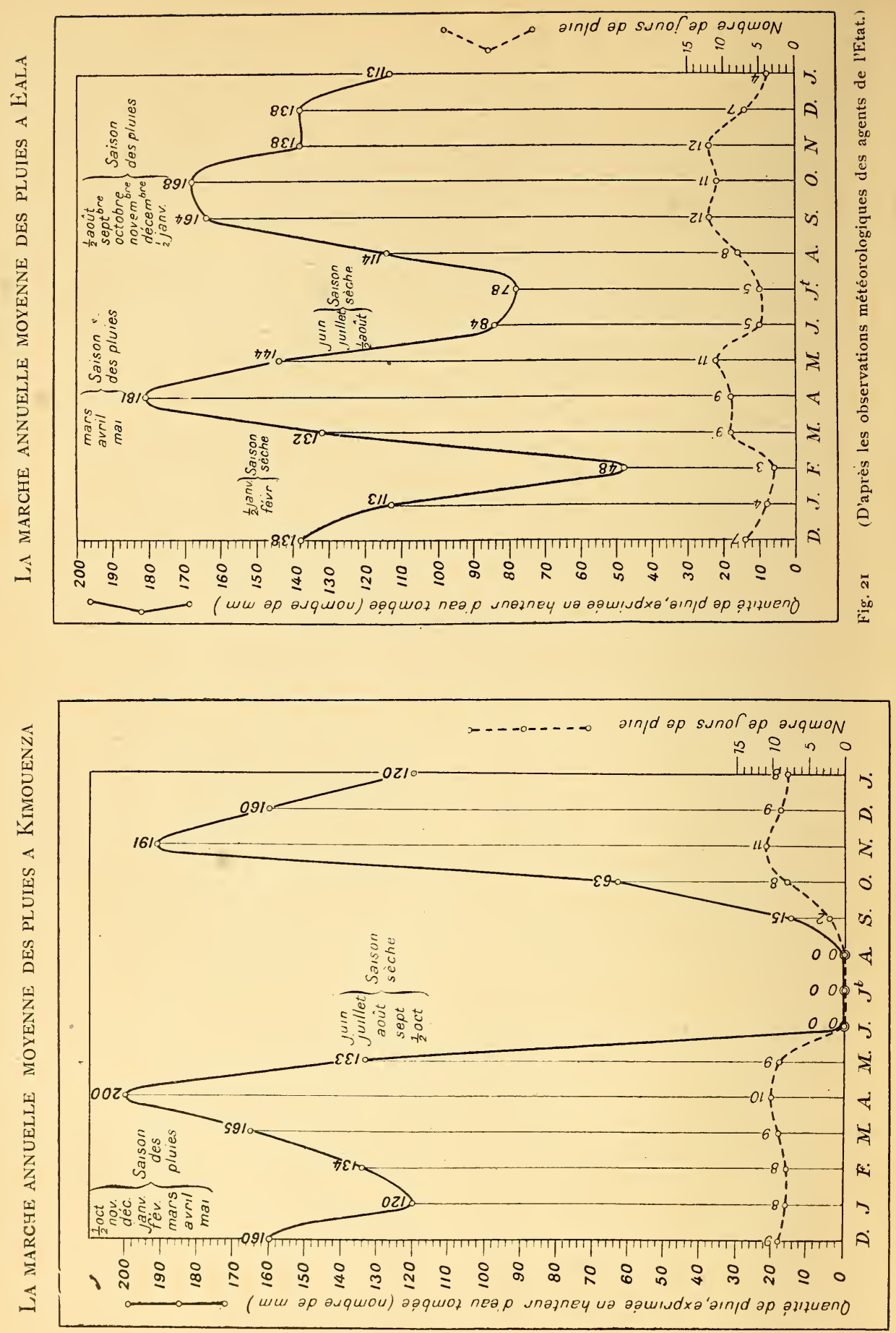
LA MARCHE ANNUELI.E MOYeNNE DES PLUIES A Loukafou.

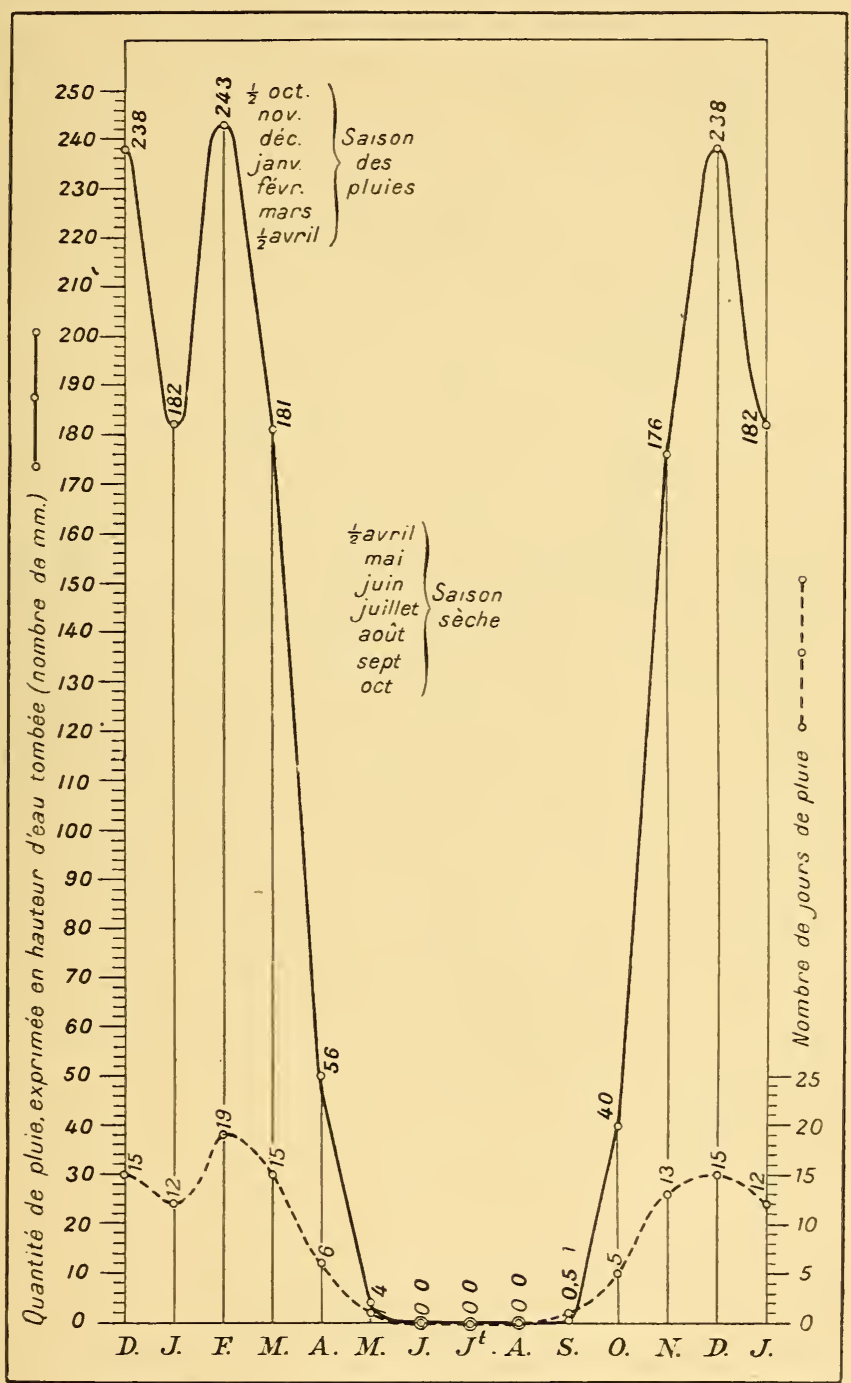

Fig. 22

(D'après les observations météorologiques des agents de l'Etat) 
La marche annuelle moyenne des pluies a Mono (Oubanghi)

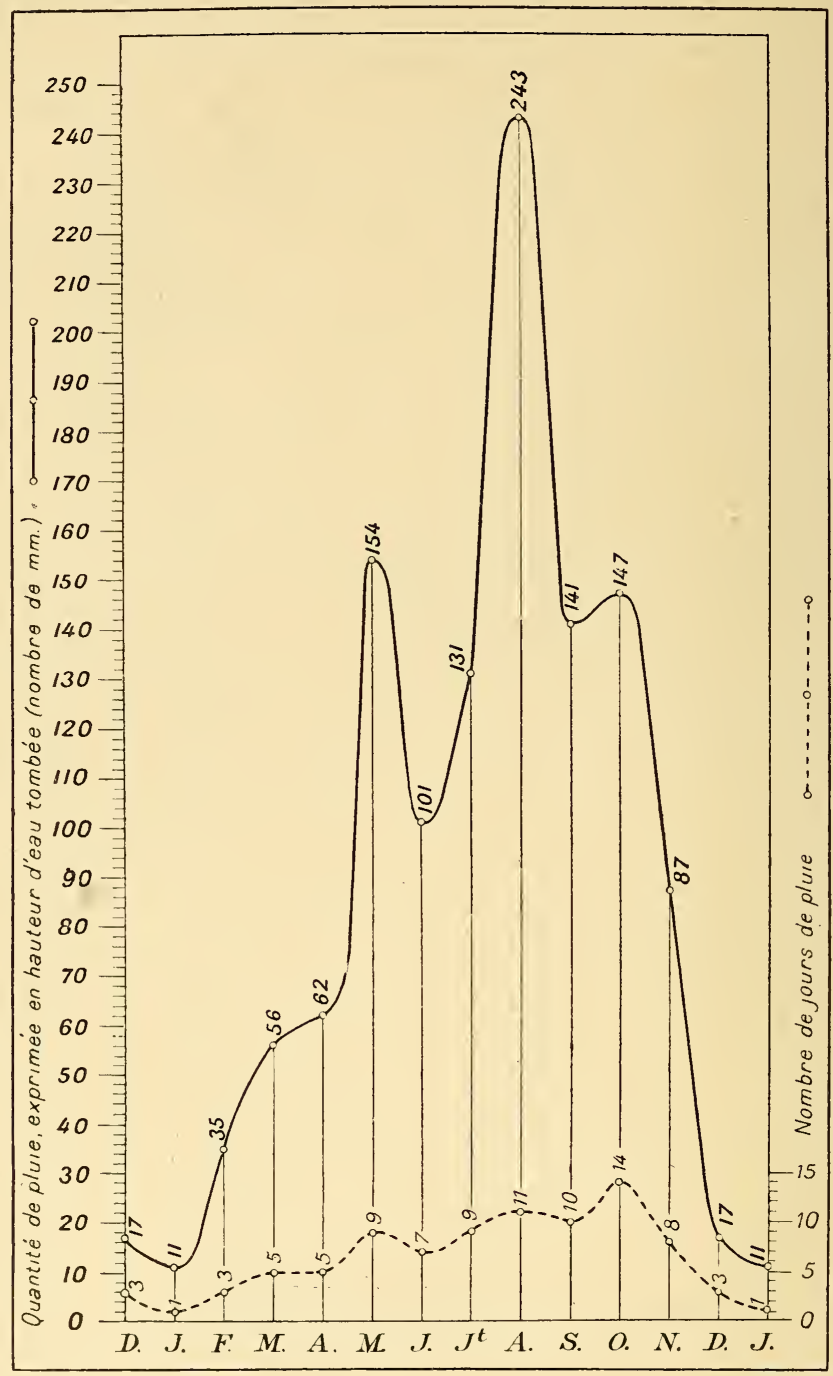

Fig. 23 (D'après les observations météorologiques des agents de l'Etat.) 
année, elle n'est excessive nulle part. En aucun endroit, elle n'atteint deux mètres de hauteur.

En résumé et en général :

$I^{\circ} \mathrm{Au}$ sud du $2^{\ominus}$ parallèle sud la saison des pluies débute

LA RÉPARTITION ANNUELIE DE LA PLUIE

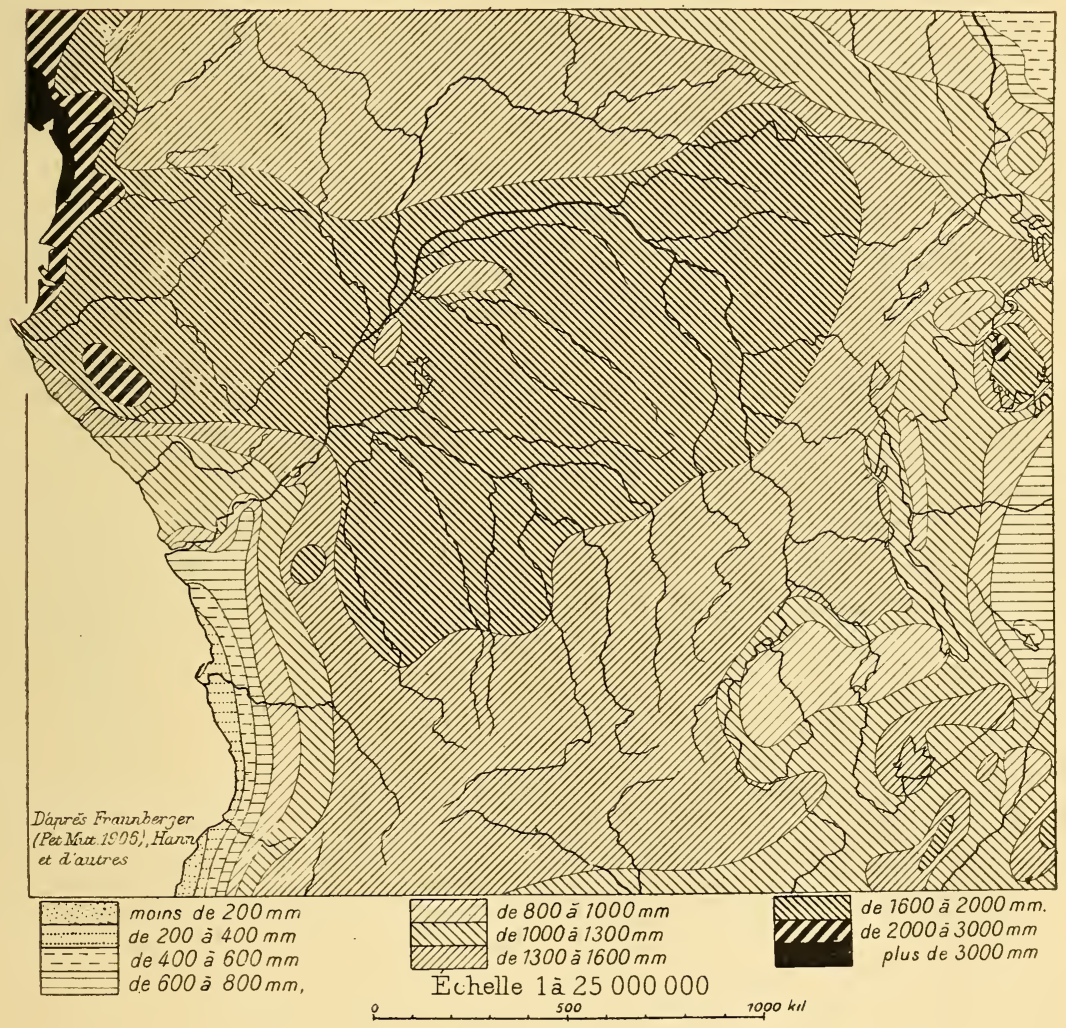

Fig. 24.

au commencement d'octobre et prend fin au 15 mai. Au nord $\mathrm{du} 2^{\mathrm{e}}$ parallèle nord, l'inverse se produit.

$2^{\circ}$ L.es mois les plus pluvieux sont novembre et décembre, mars et avril. 
$3^{\circ} \mathrm{Au}$ fur et à mesure qu'on s'avance dans l'intérieur et qu'on s'approche de l'équateur, les saisons se différencient de moins en moins, l'année entière est plus ou moins pluvieuse.

$4^{\circ}$ La grande forêt est élément actif dans le régime de répartition des pluies sur toute la région du milieu.

$5^{\circ}$ La saison chaude est celle des pluies et de plus grande humidité de l'air; la saison froide (ou de moindre chaleur) est celle du manque de pluie et de plus faible humidité de l'air. On dit de préférence saison des pluies et saison sèche.

Les vents dominants dans le Bas Congo sont ceux d'entre ouest et sud-ouest. Vers l'intérieur ce régime disparaît et sur le moyen et haut Congo, règne l'alisé du sud-est.

Tout brusque changement de milieu pour l'homme amène des perturbations organiques. Aussi le climat congolien caractérisé plus haut dans ses points essentiels, doit apporter pour l'Européen des ruptures dans cet équilibre appelé santé. Mais avec des règles d'hygiène et de prophylaxie rigoureuses, l'homme sain se fera petit à petit à son nouveau régime, gardant toute l'énergie nécessaire pour accomplir la tâche qu'il s'est engagé à remplir.

IV. - LES RIVIÈRES. - La configuration, la structure et la nature ' du sol, le régime des pluies qui affectent l'Afrique équatoriale entraînent une grande richesse dans la circulation des eaux.

Le Congo.- Réparti au nord et au sud de l'équateur, le bassin du Congo a toujours quelqu'une de ses parties en période de maximum de pluie. Ces circonstances assurent au fleuve un débit assez régulier et très abondant qui le classe au second rang des fleuves du monde. Long de 4,640 kilomètres, il draîne une surface de 3,690,000 kilo- 
mètres carrés et a un débit évalué en moyenne à 60,000 ou 80,000 mètres cubes à la seconde.

L.es affluents mèmes du Congo sont des artères puissantes.

ÉtendUES COMPARÉES DES RÉSEAUX HYDROGRAPHIQUES DU CONGO ET DE LA MEUse A L'ÉCHELle DE I A 25,000,000

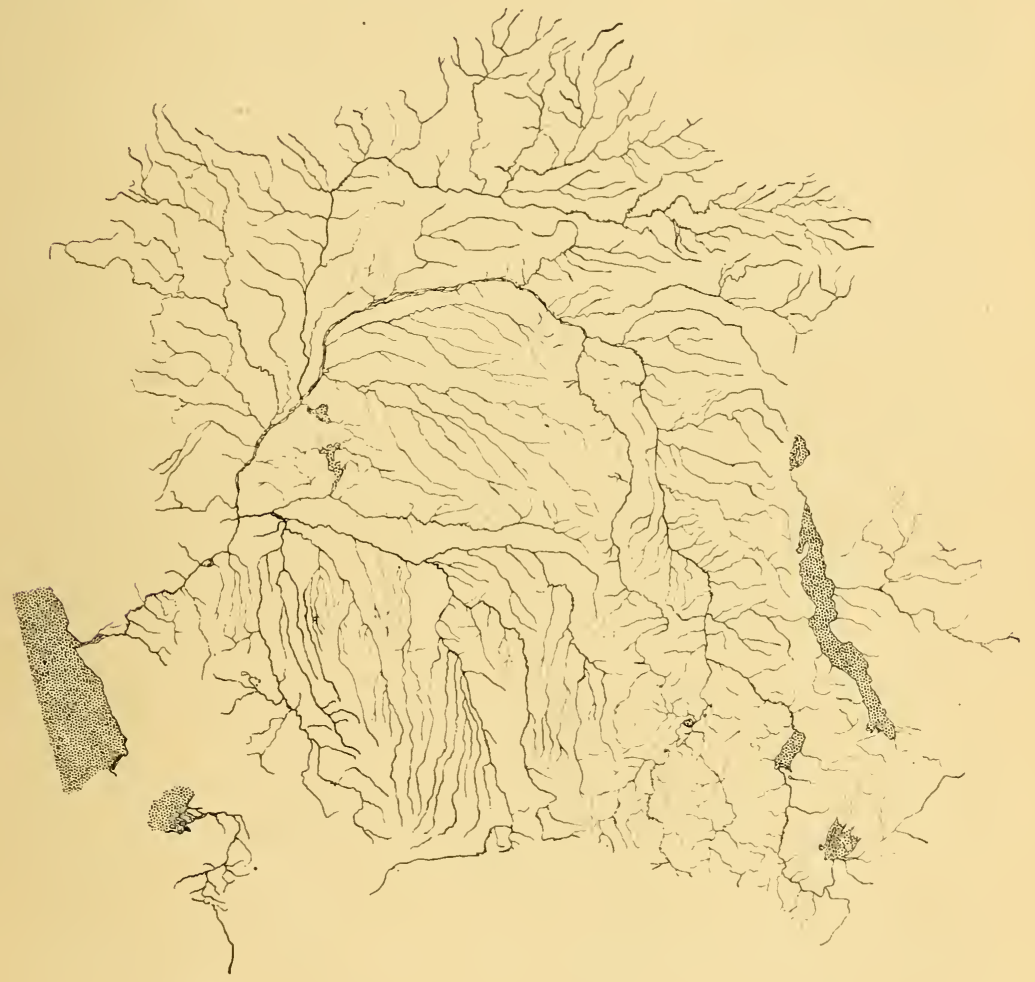

Fig. 25

Le Kasaï déverse au maître fleuve I I,000 mètres cubes à la seconde, l'Oubanghi 8,000, l'Arouwimi 4,000, le Lomami 5,000, alors que notre Escaut à Anvers ne roule par seconde que quelques centaines de mètres cubes. La largeur de l'Escaut ̀̀ Anvers est de 440 mètres à marée haute, celle de l'Oubanghi 
à son confluent avec le Congo est de 3,250 mètres, celle du Kasaï à Kwamouth 640 (confluent, partie retrécie), celle de l'Arouwimi à Basoko (confluent) I,500, celle du Lomami à Isangi (confluent) 900, celle du Congo à Banana I I,000.

Dès le cours supérieur des rivières, sur les plateaux du sud et du sud-est, la richesse du réseau hydrographique s'accentue.

Le haut plateau du Katanga est draîné par quatre artères

Longuejrs Comparées Des six PLUS GRANDS FLeUVES DU MONDE

Missouri-Missıssıppı $6.730 \mathrm{~km}$.

NiJ $6.470 \mathrm{~km}$.

Fl. des Amazones $5.570 \mathrm{~km}$.

Yang-Tsé-Kiang $5.300 \mathrm{~km}$.

Yénisséi $5.200 \mathrm{~km}$.

Congo $4.640 \mathrm{~km}$.

Fig. 26.

principales : le Louboudi (Kouléchi), le Loualaba, véritables têtes du Congo, et la Loufira qui, après un cours tantôt étalé, tantôt engorgé et parsemé de chutes, se rejoignent dans la. plaine des lacs Oupemba et Kisalé. C'est dans cette plaine, après les rapides de Kondé, que le Loualaba dont le nom restera au fleuve dans tout son cours supérieur, présente la première section navigable. En aval, le Loualaba reçoit les eaux de la quatrième grande artère du Katanga, le Louapoula qui, sous différents noms, s'épanche en deux grands lacs : Bangwélo et Moéro et est affecté d'un régime analogue à celui des autres rivières du Katanga. 
Le Loualaba, large de 500 mètres en moyenne coule vers lt nord jusqu'aux chutes Stanley (Stanley-falls) à partir desquelles on le dénomme Congo. Sa navigabilité est interrompue des chutes des Portes d'Enfer à celles de Chambo. Il a reçu le trop plein des eaux du fossé Kivou-Tanganika par la Loukouga.

La série des chutes Stanley constitue la transition du Loualaba au Congo moyen appelé souvent haut Congo (des dites Stanley-falls au confluent du Kasai).

C'est ce Congo moyen qui dans la vaste plaine du milieu forme la partie principale du cours entier du fleuve. Dans son large lit viennent se déverser les eaux des principaux collecteurs secondaires du bassin, à gauche le Lomami, la Loulonga, le Rouki, le Kasaï (sous affluents : Louloua, Sankourou, Fini à droite; Loanghé, Kwango à gauche); à droite : l'Arouwimi, l'Itimbiri, La Mongala, l'Oubanghi, l'Ouellé, la Sanga (Congo français). C'est dans cette plaine que l'épanchement fiuvial atteint toute son ampleur: nappe liquide de 20 kilomètres de largeur totale se mouvant parmi une infinité d'îles en un réseau encore mal connu.

Quittant la vaste plaine centrale où çà et là on retrouve des restes de l'ancien épanchement lacustre — lac Toumba, lac Léopold II, Stanley-Pool, étalement des eaux fluviales en nombreux marais - le Congo perce les rangées des montagnes côtières. C'est cette trouée qui constitue la transition du cours moyen au cours inférieur.

Dans la région des chutes, au fond des gorges qu'il érode sans cesse, le Congo se resserre jusqu'à 400 mètres et moins; il roule sur une profondeur considérable avec une rapidité qu'on peut s'imaginer si l'on considère que le débit par seconde peut varier de 50,000 à I20,000 mètres cubes. Du Stanley-Pool au pied des escarpements tabulaires atlantiques, c'est-à-dire sur un parcours de 360 kilomètres, les eaux congoliennes franchissent une dénivellation de 220 mètres et 
forment la série des 32 rapides ou chutes dites de Livingstone, dont les principales sont celles de Zinga et les dernières celles de Yellala. A Matadi, où le fleuve redevient navigable, sa. largeur est d'environ I,440 m., sa profondeur

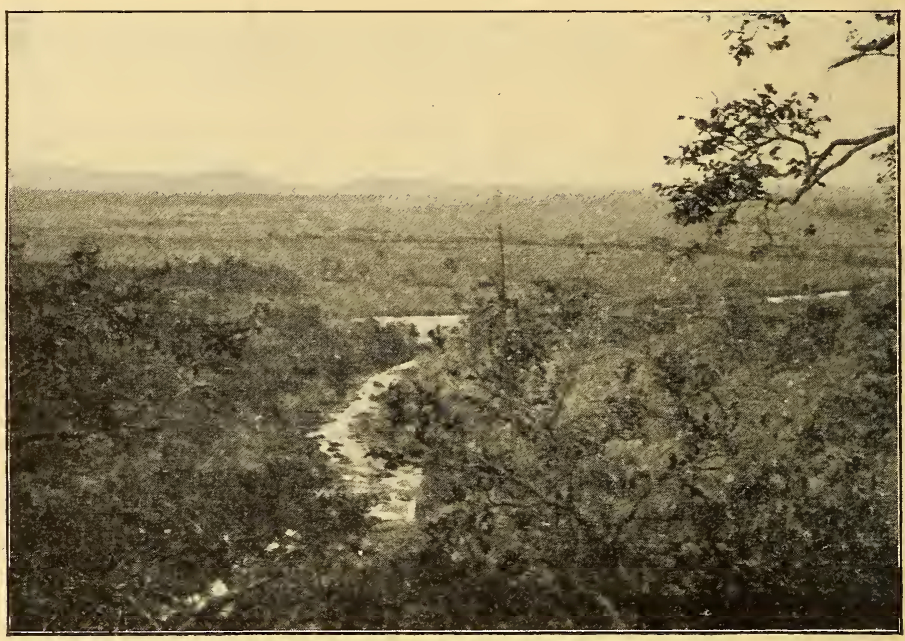

Fig. 27. - Haute plaine du loualaba. - La rivière s'engage pár se goulot du Nzilou ou Nzilo à la traversée des Mitoumba dont les collines M'poungwé sont les premiers remparts. Cette photographie a été prise du point de vue A renseigné sur le croquis cartographique ci-contre. Le champ de l'objectif est indiqué sur ce croquis cartographique par les droites $\mathrm{AB}, \mathrm{AC}$. - Comparer attentivement la photographie et la carte. - « Les collines M'poungwé s'ouvrent suivant une fente de direction S.E.-N.W.; par cette fente le lac de jadis a trouvé un écoulement et le Loualaba actuel s'est formé. - La rivière ou si l'on veut le fleuve arrive en méandres marqués, à travers une plaine marécageuse en ce moment de l'année - juillet - juste en avant du goulot où il va s'engager, le Loualaba s'épanouit en un petit pool à grosses roihes où crocodiles et hippopotames se complaisent. » (Ext. carnet de route manuscrit Ch. Lemaire, Expédit. scient. du Katanga.) - (Phot. Lemaire).

Passer à la photographie suivante.

de $70 \mathrm{~m}$. A 3 kilomètres en aval, il s'engorge de nouveau, se resserre à $560 \mathrm{~m}$., mais affouille son lit à une profondeur de I 20 mètres. La coulière fluviale s'élargit un peu, la profondeur des eaux diminue. A une trentaine de kilomètres en aval de Matadi, l'estuaire s'annonce.

A Boma, le grand fleuve a repris son ampleur. 
Sa profondeur varie en raison de ses apports détritiques qui font varier l'emplacement du chenal principal. Celui-ci afteint fréquemment I2, I5, 20 mètres de profondeur. La

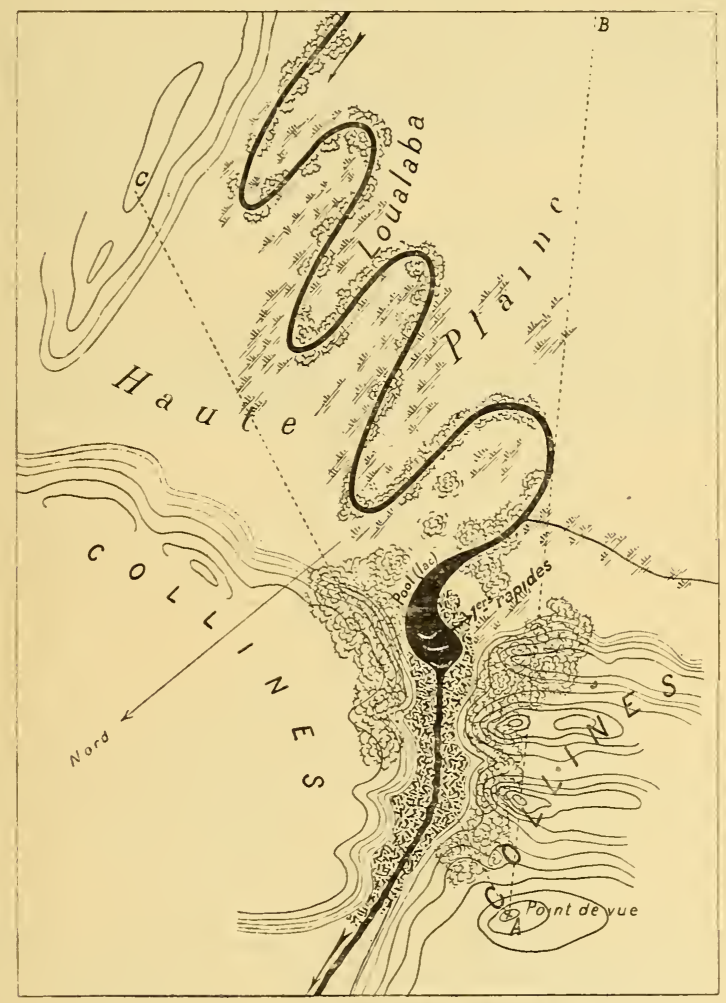

Fig. 28. - La haute plaine du loualaba et l'extree du goulot du NZilou à l'échelle de I à r route manuscrit).

courbe de 35 mètres remonte à plus de 25 kilomètres en amont de Banana. A l'issue du lit fluvial à la mer, en face de la pointe de Banana, le plomb accuse l'existence d'un fond de 446 mètres. Arrivée à la mer, l'énorme masse des eaux congoliennes entretient un estuaire sous-marin que révèle claire- 


\section{$-50-$}

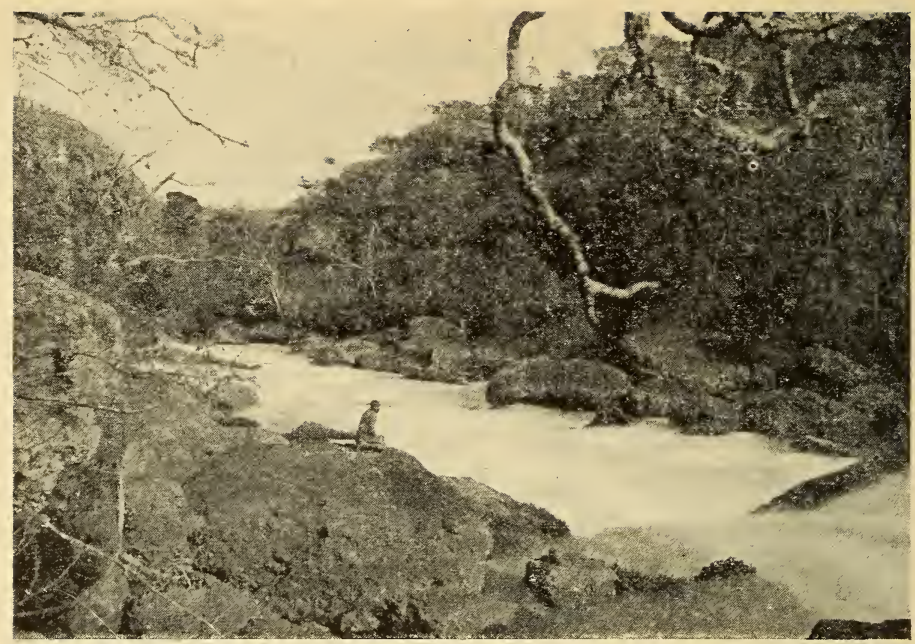

Fig. 29. - Le goulot du N'zilou. - $~ \propto$... Puis la rivière commence à dévaler sur les énormes blocs de roches (poudingues quartzeux) qui forment un éboulis géant où l'eau se baratte en une perpétuelle lessive écumante; la largeur du goulot se rétrécit jusqu'à une quinzaine de mètres par places; les parois sont formés d'abord par des éboulis de roches, puis par la crevasse mème des M'poungwé. » (Lemaire - ibid.)

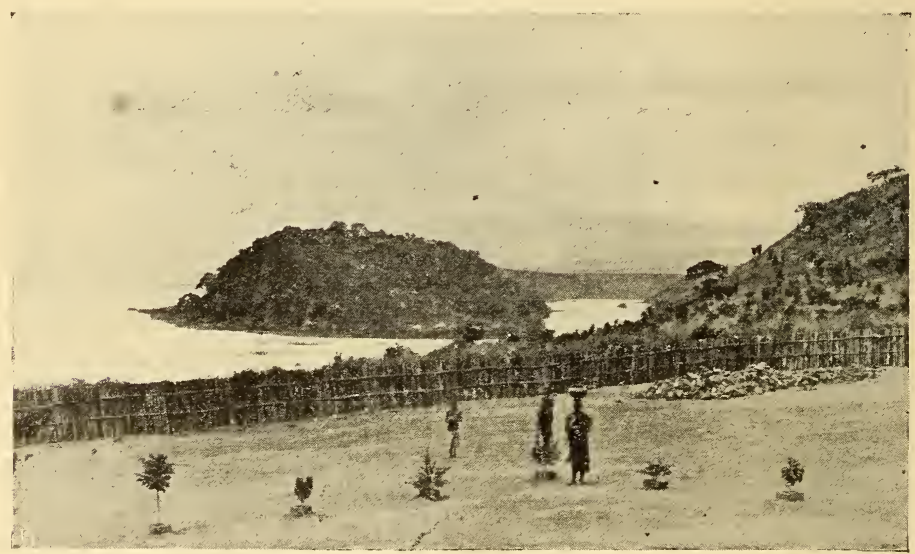

Fig. 3O. - Le goulot de SORTIE du LAC Mó́ro: le Louapoula

(Phot. Lemaire.) 
ment sur nos cartes l'allure des courbes de profondeur. Elle est emportée vers le nord-ouest par le grand courant marin

Confletext du Congo et de l'Oubangh

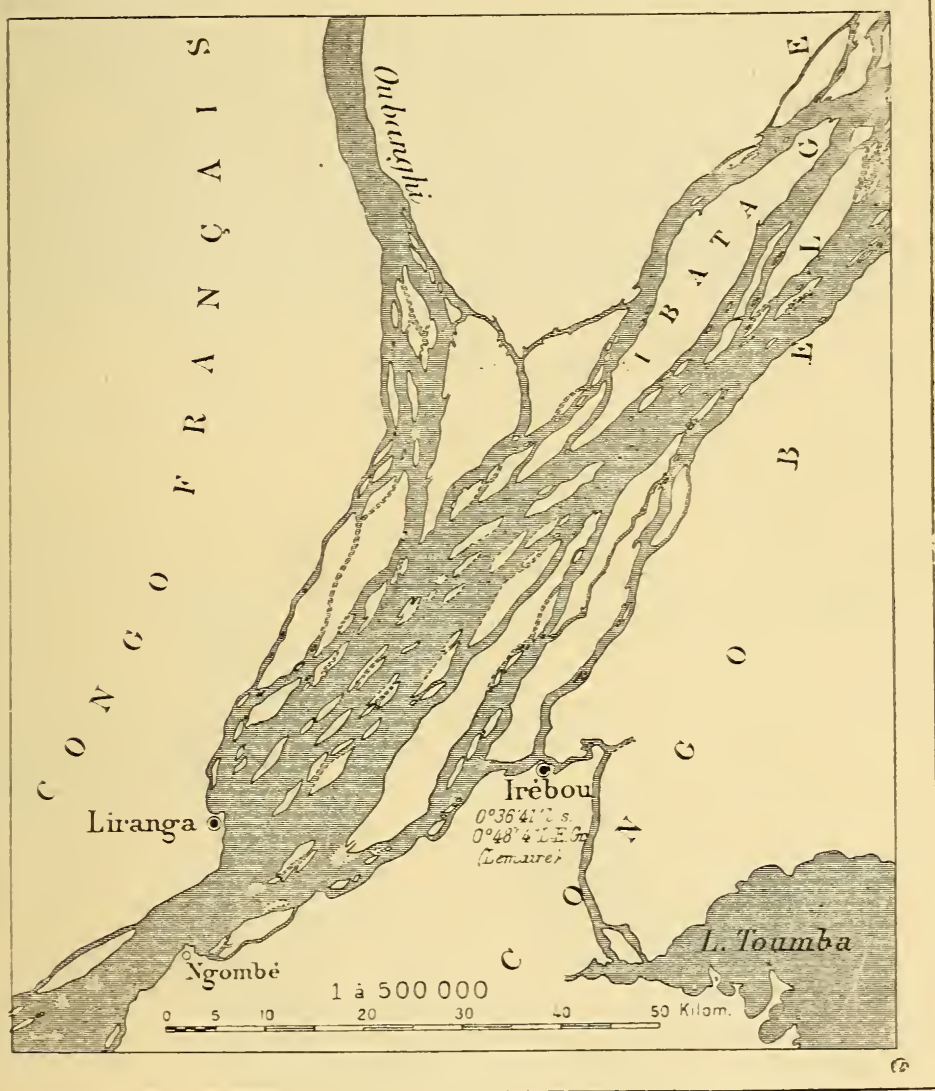

Fig. $3^{1}$

(D'après Grexfeln Carte du fleur'e Congo des Stanley-Falls au Stanley-Poul, à l'échelle du $250,000^{\circ}-$ Geogr. Journal, nov. 1902.)

froid renant du sud et ce n'est qu'à 25 ou 30 kilomètres au large de l'embouchure que les eaux douces et chargées 


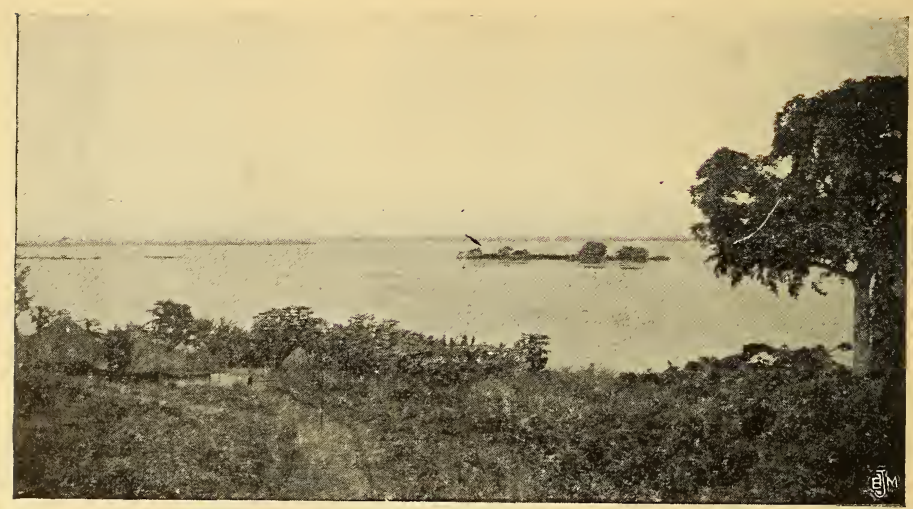

Fig. 32. - Stanley-Pool; - a l'horizon la rive française.

(Phot. communiquée par le Ministère des Colonies.)

Stanley-Pool.

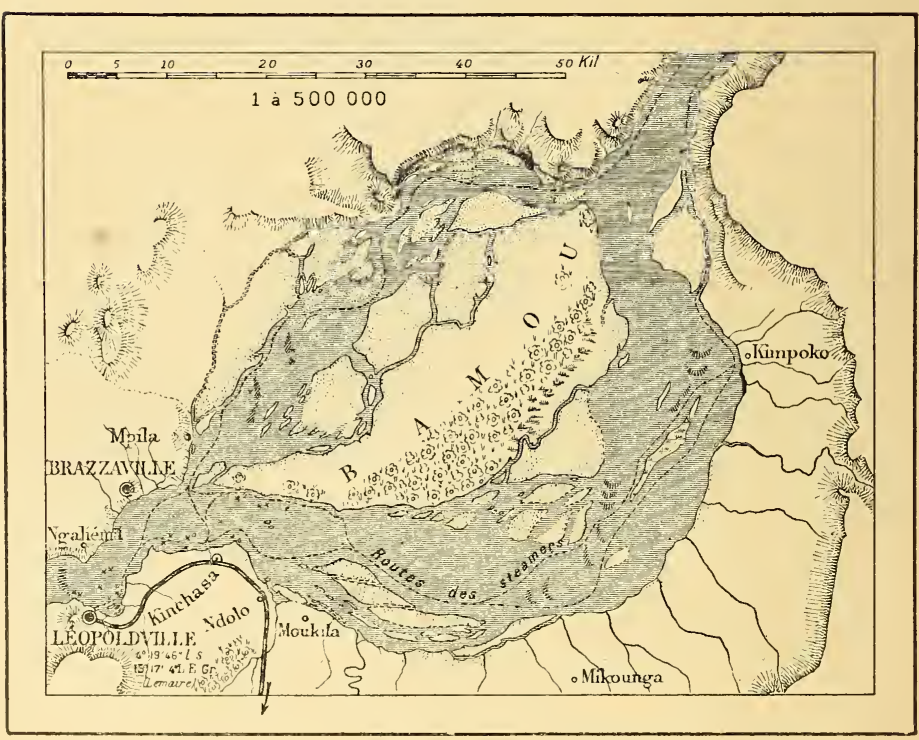

Fig. 33.

(D'après GRFFELI, ib:d) 
du fleuve africain sont définitivement mêlées à la vague saline de l'océan. La puissance de ce courant d'eau s'ouvrant à la mer est telle que la force de la marée est détruite et que l'estuaire est entièrement libre de toute barre.

\section{Le. Bas-Congo.}

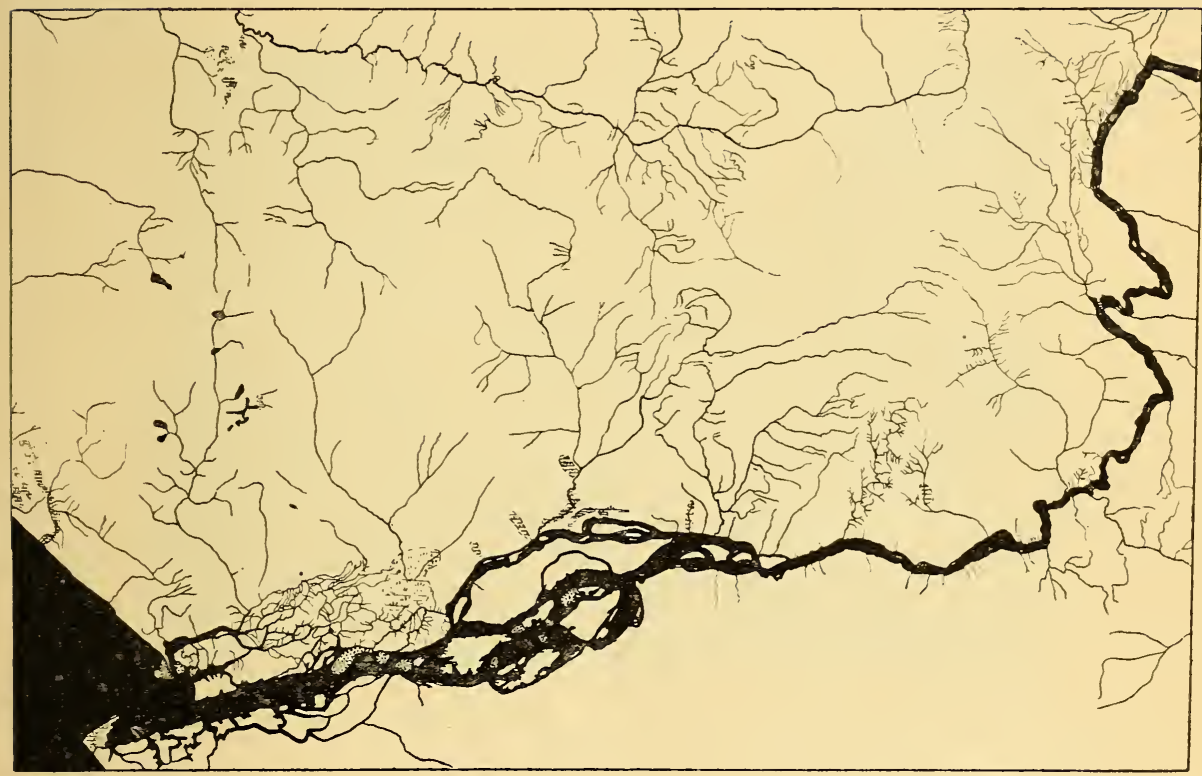

Fig. 34. - Echelle de 1 à 1.000.000. (D'après la Carte au 100.000 du Bas-Ccngo par H. Droogmans)

Le Nil. - Quoique, en réalité, la limite nord-orientale de la colonie belge soit la ligne de faîte Congo-Nil, nous possédons un débouché sur la voie du Nil grâce à des conventions établies entre l'Angleterre et le Souverain Léopold en I897. Par le lac Albert-Édouard et la Semliki, le Nil draîne une faible partie de nos territoires. Il est important comme route d'accès et de débouché malgré l'encombrement de son lit par une végétation touffue flottante ou fixe, le sedd, qui 
rend la navigation difficile. Du lac Albert à Doufilé, le fleuve est très praticable. De Doufilé à Redjaf, il est barré par des rapides. En aval de Redjaf il ne pourra constituer une bcnne voie fluviale qu'après avoir été bien aménagé. L'importance du Nil pour la Congolie est toute économique. Nous aurons l'occasion d'en reparler plus loin.

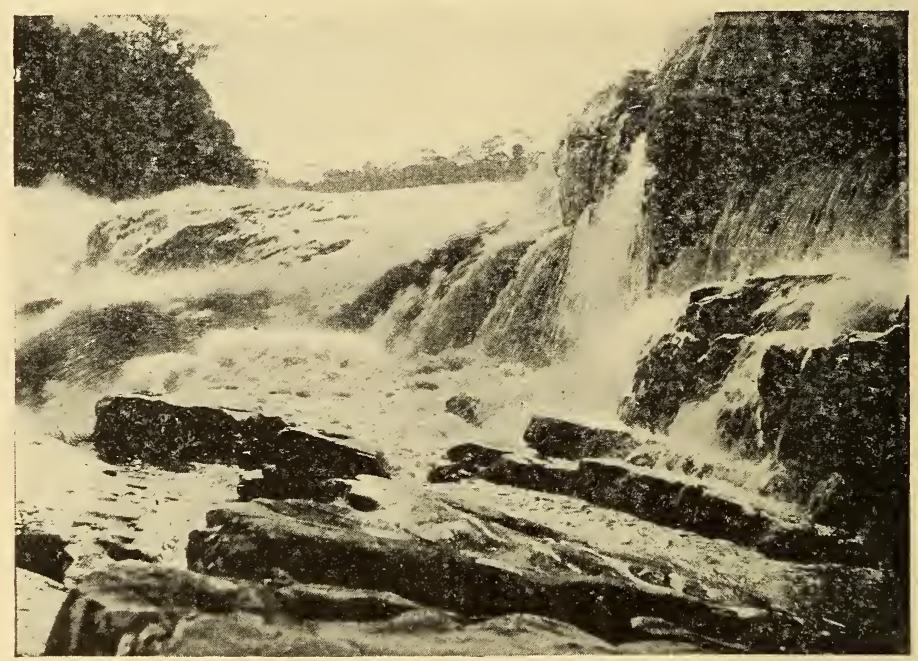

Fig. 35 - La rivière TÇно po saute sur un gradin du rebord abrupt séparant l'arrièreplateau congolien de la plaine centrale. (Voir étude du relief, $3^{\circ}$.)

Le régime des eaux. - Pour des raisons dont nous nous sommes partiellement rendu compte, les eaux du Congo ne sont pas affectées de crues subites. Ses affluents dont les surfaces de draînage sont soumises à un régime de pluies périodiques, sont affectés de tortes crues périodiques. Cela se produit dans les régions éloignées de l'équateur : les débits du Loualaba, du Lomani, de l'Oubanghi, de la Sanga, du Kasai subissent ainsi de fortes oscillations. Mais en vertu de l'alternance des saisons au nord et au sud de l'équateur, 
les crues de l'Oubanghi et de la Sanga alternent avec celles du Kasai, du Lomami, du Loualaba, ce qui apparemment proroque deux montées du niveau du bas Congo, la première due aux affluents de droite (nordj, la seconde aux affluents de gauche (sud). Sur les pays de l'Oubanghi, la saison des pluies se produit de mars à novembre (voir diagramme des pluies à Mono) et provoque une crue fluviale qui se marque dans le bas Congo en avril, croît jusqu'en octobre, époque à laquelle elle est maxima. Quand l'apport de l'Oubanghi et de la Sanga baisse, c'est-à-dire en fin octobre ou début de novembre, le débit du Kasaï, du Lomani, du Loualaba commence à monter. En effet, la saison des pluies s'étend d'octobre à mars ou mi-avril sur les régions méridionales de la colonie (voir diagramme des pluies à Loukafou).

Si tous les affluents du nord rendaient en même temps au maître fleuve leurs tributs de crues périodiques et si de même tous les affluents du Sud dégorgeaient ensemble dans le collecteur Congo l'apport saisonnier de leurs eaux maximales, nous assisterions à des crues alternantes très régulières du bas fleuve. Mais non seulement les discordances de temps sont considérables dans l'apport au fleuve de Léopoldville des eaux lointaines du Loualaba (Katanga) et du Kasaï, du haut Oubanghi et de la Sanga, mais encore le débit uniforme de toutes les rivières équatoriales - Loulonga, Rouki, Arouwimi - peu sujettes aux crues et aux maigres en raison du régime des pluies (voir diagramme des pluies à Éala) est un facteur de régularisation du niveau des eaux du Congo. Ajou-. tons encore que la différenciation du degré de perméabilité des sols, que la variété du relief et du tapis végétal, que l'existence de bassins lacustres régulateurs du débit fluvial viennent singulièrement modifier les premiers éléments du problème. Simple en apparence, l'étude du régime d'un fleuve est très complexe et il serait prématuré de vouloir apporter beaucoup de précision dans l'exposé hydrographique du bas- 
sin du Congo. Nous pouvons en général dire que dans le bas Congo une première montée des eaux, provoquée par les pluies du bassin de l'Oubanghi, se marque aux mois de novembre et de décembre. Les pluies sur la surface de ruissellemen'́ ḋ̀s affluents de gauche (partie sud de la colonie) provoquent aux mois de février et de mars une seconde montée qui perdure assez longtemps en raison cie l'éloignement des lieux d'origine des eaux de crues (Katanga). Cette dernière crue atteint son maximum dans le bas Congo en mai. Le niveau du fleuve baisse ensuite assez rapidement. Le moindre niveau moyen se marque en septembre.

L'examen du bassin du Congo nous fait entrevoir tout l'avantage que l'on peut retirer de l'existence de ce formidable réseau fluvial. Il est vrai que des séries de chutes ont opposé à la pénétration une barrière longtemps infranchie, mais le chemin de fer, comme nous le verrons tout à l'heure, en a $\mathrm{eu}$ raison et le système de navigation fluviale du bassin congolien surpasse en étendue tout autre réseau analogue du monde à l'exception de celui du fleuve des Amazones. Cette valeur est augmentée de l'existence de trois ports maritimes sur l'estuaire : Banana, Boma, Matadi dont le dernier est situé fort avant dans les terres, à plus de 125 kilomètres de la pointe de Banana.

V. - LA VÉGÉtATION. - Le climat qui affecte le bassin $\mathrm{du}$ Congo peut se résumer en trois termes : $\mathrm{I}^{\circ}$ lumière violente; $2^{\circ}$ température élevée à faibles variations; $3^{\circ}$ humidité atmosphérique entretenant par une copieuse précipitation une très riche circulation aqueuse à la surface comme à l'intérieur du sol. De telles conditions s'accordant sur un sol d'altitude faible ou modérée doivent forcément entretenir un puissant tapis végétal. L'exubérance de la vie végétale est en effet une des caractéristiques des pays congos.

Là où il fait toujours humide la végétation a pris l'aspect 
de la grande forêt dense toujours veite. La forêt vierge congolienne couvre toute la grande plaine du milieu et présente des éclaircies dont la dimension varie avec l'abondance de la circulation aqueuse. En général, les grandes clairières se

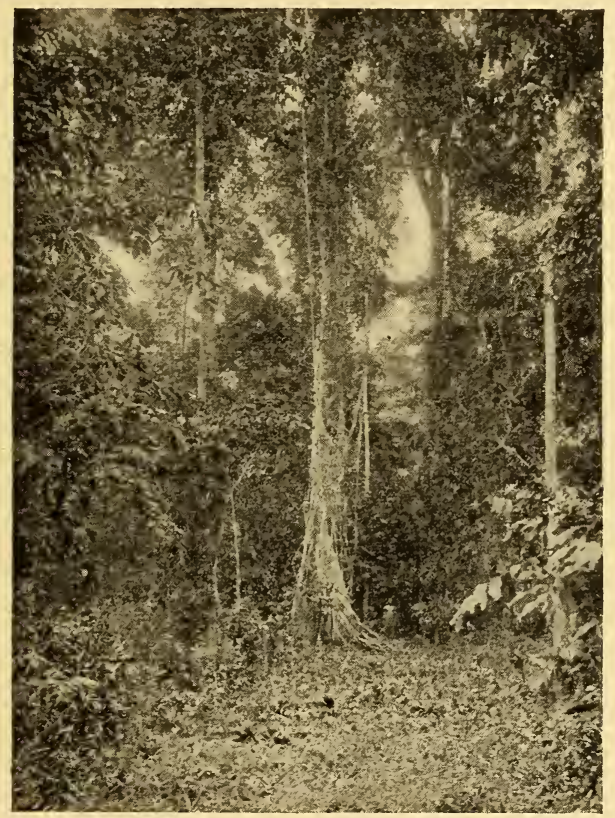

Fig. 36. - Une ÉClaircie dans la Forêt dense. (Considérer la dimension du personnage.)

trouvent sur les parties les plus élevées de la plaine, dans les zones de contact des surfaces de ruissellement. La forêt remonte en galeries profondes dans les coulières des cours d'eau, notamment dans celles du bassin du Kasaï.

D'une activité constante, sans période de repos dû à un abaissement de température ou au manque d'eau, cette végétation forestière vit en somme plus fort et plus longtemps 
que celle de nos pays tempérés et atteint de gigantesques proportions. L'excellence des conditions naturelles a entraîné une très grande variété de la flore en sorte que, à la différence des bois de nos régions qui présentent souvent une essence dominante, la forêt congolienne compte, même sur un espace restreint, un très grand nombre d'espèces diverses.

Stanley, en des pages devenues classiques, nous a décrit la sylve congolienne:

"Imaginez, dit-il toute la France et toute l'Espagne revêtues d'arbres d'une hauteur variant entre 6 et 54 mètres; les cimes de ces fûts, dont le diamètre mesure de quelques pouces à 120 centimètres et plus, sont tellement rapprochées, qu'elles s'enchevêtrent et empêchent de voir le ciel et le soleil. Lancez d'un arbre à l'autre des càbles épais de 5 à 40 centimètres, contournezles, plaquez-les contre les troncs, ou enroulez-les tout autour et jusqu'aux sommets comme un serpent sans fin. Prodiguezleur les feuilles et les fleurs, et que là-haut ils aident les ramures à cacher le soleil : des branches les plus élevées, qu'ils retombent par centaines à quelques pieds du sol... Que sur chaque fourche, sur chaque branche horizontale, s'élèvent des choux géants, et ces végétaux à larges feuilles ensiformes qu'on appelle la plante à oreilles d'éléphants, puis des touffes d'orchidées, merveille des tropiques, et une draperie de ces délicates fougères, si communes dans la grande forêt; couvrez branches, rameaux, lianes, de mousses épaisses, ressemblant à une verte fourrure. Une fois chaque arbre en place avec sa parure de lichens et de sarments, il ne reste plus qu'à étendre sur le sol un tapis verdoyant de phryniums, d'amomes et de buissons nains. Voilà la grande forêt, la sylve antique et compacte. »

" Nous gagnâmes la Mitammba, cette forêt sinistre que nous avions en face de nous depuis notre départ; et, disant adieu au soleil, nous entrâmes dans ce bois redouté. Accoutumés à une marche rapide, nous devions nous arrêter sans cesse, attendre avec patience qu'on pût faire quelques pas. Pendant ce temps-là, les arbres nous versaient leur rosée, chaque feuille pleurait sur nous; et, de toutes les branches, de toutes les lianes, de toutes les tiges, l'eau nous arrivait en larges gouttes. Au-dessus de nos tétes, des lits de rameaux enlacés nous cachaient la lumière. 
Nous ne savions pas si le jour était clair ou sombre, ensoleillé ou brumeux. Nous marchions au milieu d'un faible crépuscule, celui des climats tempérés une heure après le coucher du soleil...

« ... Le terrible sous-bois qui, à l'ombre des géants de la forêt, encombrait tout l'espace, était un miracle de végétation; c'était un inextricable fourré dont toutes les plantes se disputaient chaque

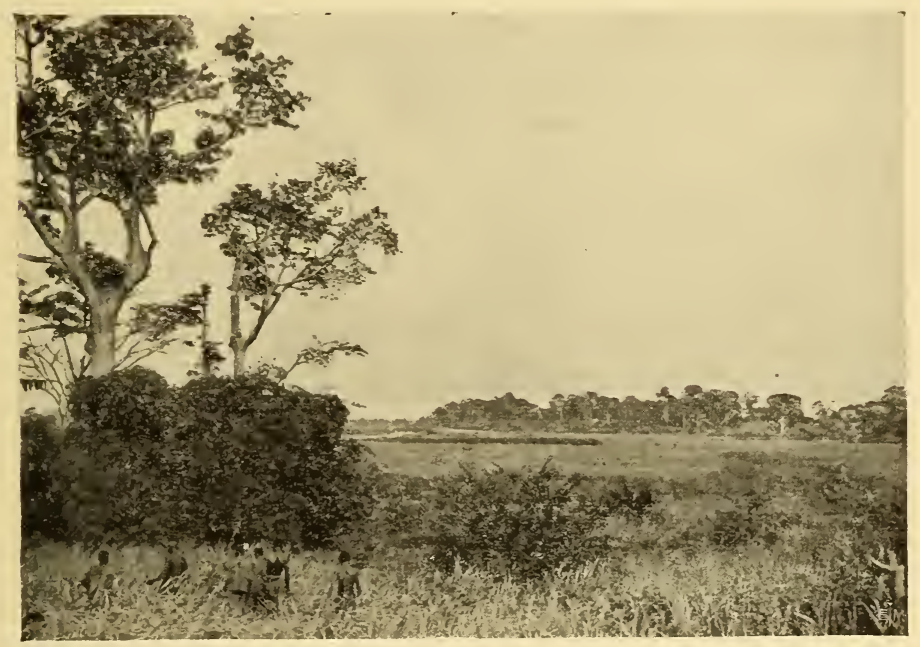

Fig. 37. - La GRANDE Savane congolienne (étendue couverte de hautes herbes, de bois de bouquets d'arbres ou d'arbres isolés).

(Photogr. communiquée par le Ministère des Colonies.)

pouce du terrain, d'où elles s'élançaient arec une luxuriance que peut seule donner cette prodigieuse serre chaude. Certes, nous avions vu des forêts auparavant, mais celle-ci devait faire époque dans notre existence, - souvenir d'une amertume à ne jamais oulllier. Tout mettait le comble à nos misères: l'obscurité des lieux, l'humidité pénétrante, l'insalubrité de l'atmosphère, la monotonie de la scène, - toujours des branches enlacées, des amas de feuillage, toujours les hautes tiges des arbres s'élevant d'une jungle éternelle, où nous avions à faire notre trouée et à passer en rampant sur les mains et les genoux... (Stanley. A travers les ténèbres d'Afrique.) 
Là cù les saisons pluvieuse et sèche alternent, notamment sur les plateaux dont l'altitude réduit et la température et la circulation d'eau, la végétation s'est adaptée, le tapis

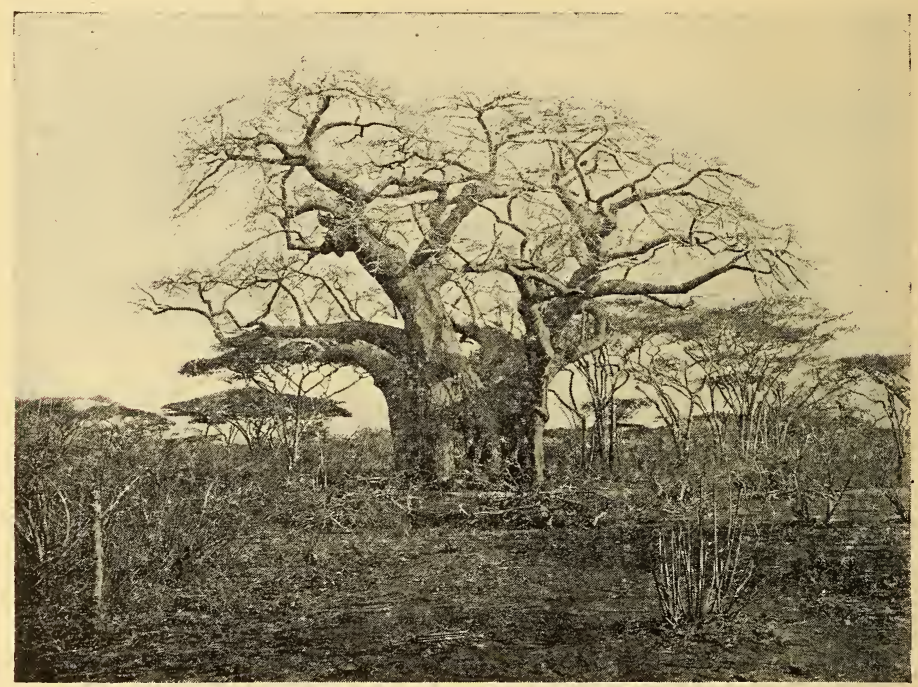

Fig. 38, - La SAVANe A BAoBab. Le baobab ou arbre à pain de singe est un arbre à feuilles caduques, feuillant avec la pluie. Son tronc, peu élevé ( 4 à $5 \mathrm{~m}$. du sol à la naissance des branches) peut atteindre 30 à $35 \mathrm{~m}$. de tour. Il est fréquent dans le Bas-Congo et disparaît au delà du Kasai. Dans l'Afrique Orientale, son domaine est étendu. Sa fibre, son écorce, sa feuille, son fruit servent à des usages multiples.

Notre gravure sur laquelle on peut voir, à l'arrière-plan, des acacias parasols, nous montre un bel exemplaire de baobab ainsi que l'aspect type de la savane à baobab, réalisé particulièrement bien en Afrique Orientale allemande.

(D'après Vegetationsbilder de Karsten et Schenck. -Gustav Fischer à Iéna.)

végétal est désormais la savane dont le type le plus caractérisé est la savane à baobab.

Comme nous le savons, le passage de la forêt vierge à la savane n'est pas brusque. Il est progressif et se marque dans l'existence de la forêt en galerie.

Lorsque le degré et la périodicité de la sécheresse s'accen- 
tuent, la savane s'appauvrit, les arbres se rabougrissent, deviennent buissonneux, le tapis végétal devient brousse.

Quelquefois même les buissons disparaissent totalement et font place à un tapis d'herbes. C'est la steppe d'ailleurs rare et de faible étendue au Congo.

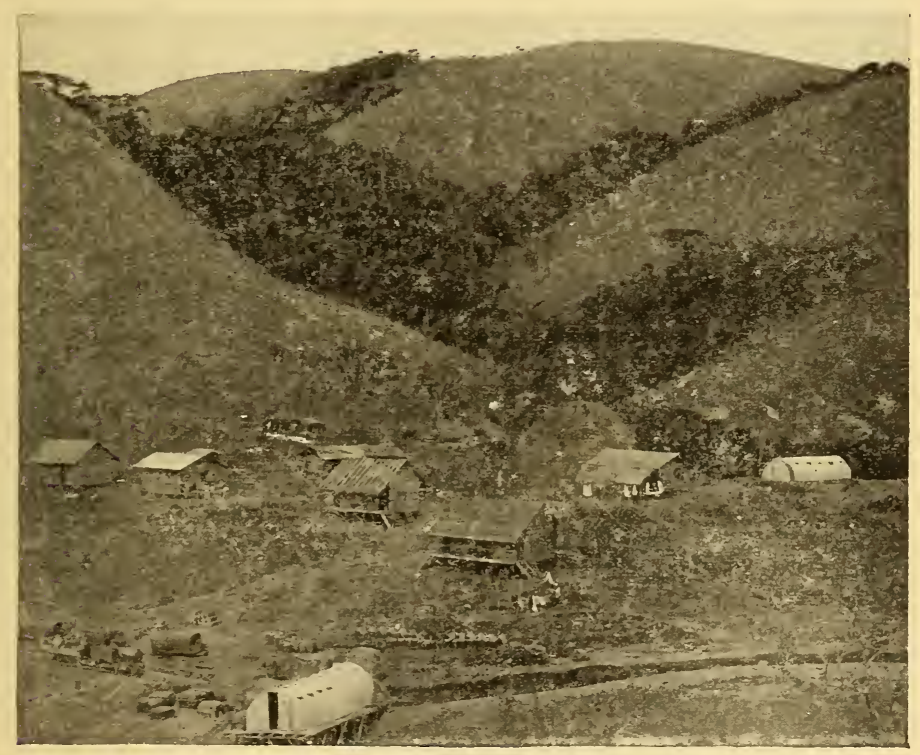

Fig. 39. - La FOrÊt EN GALERIE. - Campement de blancs au kilomètre i9 (construction du chemin de fer de Matadi à Léopoldville). - (D'après Goffin.)

Dans les parties basses comme sur les plateaux, le marais est fréquent: marais côtier, de petites dimensions, dans les anses tranquilles de l'estuaire du Congo, remarquable par sa végétation dite mangrove (forêt littorale de palétuviers), - marais fuvial ou lacustre tel que ceux du Bangwélo, du Moéro, du lac Léopold II, de l'Oubanghi, du Congo moyen, - marais éponge très fréquent sur les plateaux d'altitude 


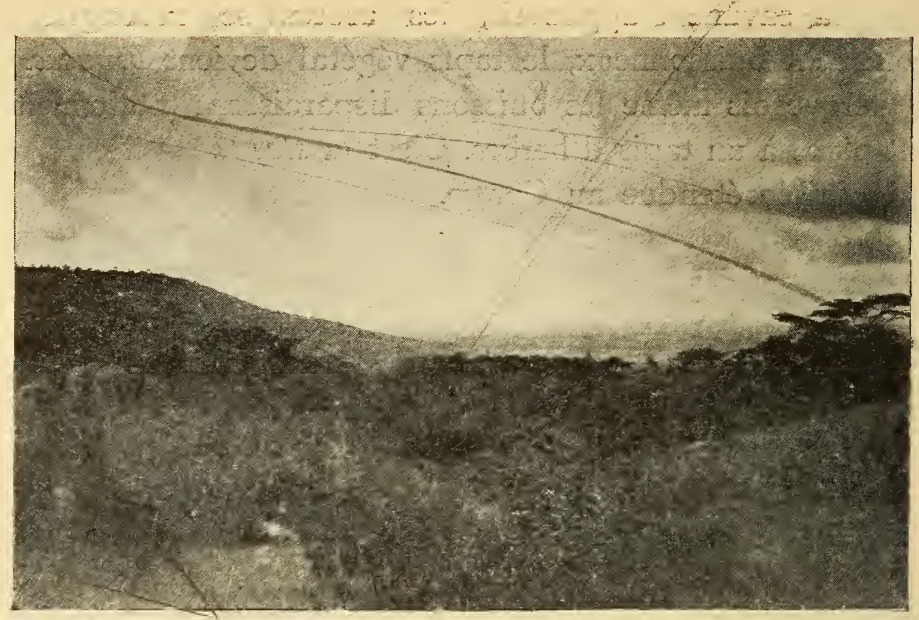

Fig. 40. - DE la Savanne a LA BRousse.

(Phot. communiquée par le Ministère des Colonies.)

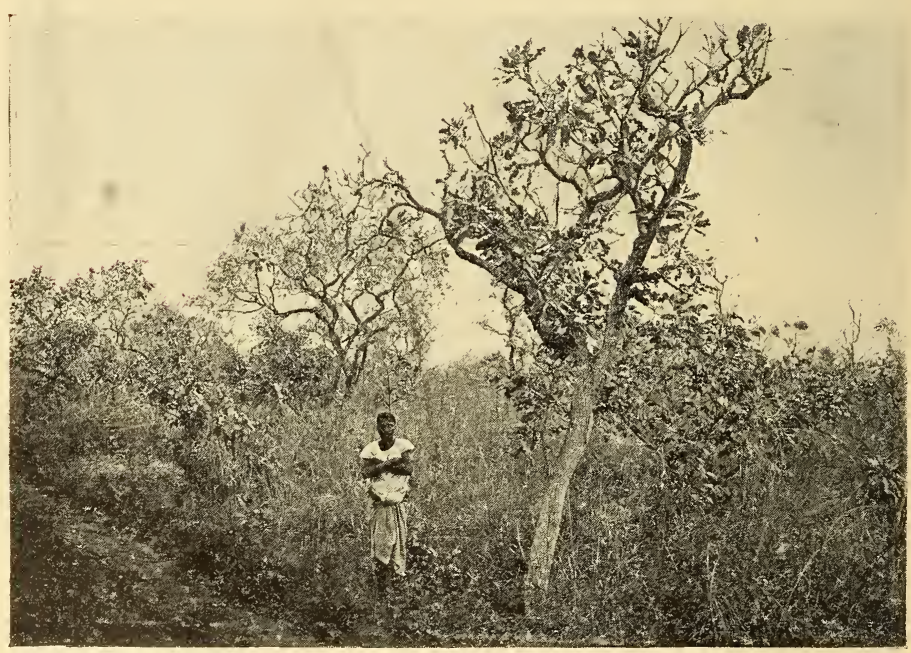

Fig. 4I. - LA BROUSSE AFRICAINE : grandes herbes, arbres rabougris ou de petite taille. - (D'après Vegetationsbilder de Karsten et Schenck. - Gustav Fischer à Iéna.) 
considérable à pente indécise (zone limite des surfaces de ruissellement Congo-Zambèze).

La flore du Congo varie évidemment suivant les régions. Si la zone centrale est remarquable par son unité, la savane et la brousse ont un aspect plus divers. On peut distinguer

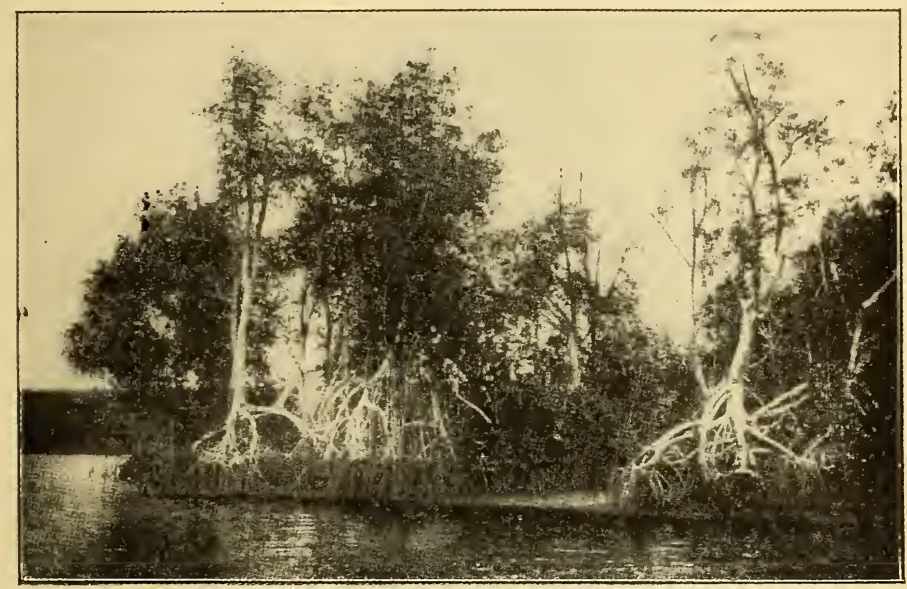

Fig. 42.- La Mangrove de l'estuaire du Congo (d'après O. Drude, Geogr. Hdb. de Sco$b e l)$. - La Mangrove est une. forèt littorale constituée par l'association de palétuviers. L'adaptation de ceux-ci au milieu maritime est surprenante. Par des racines très nombreuses, puissantes et profondes, ils se fixent solidement sur le sol inondé journellement par la mer. Les racines vigoureusement enchevètrées sont biquotidiennement baignées par le flot dans leur partie supérieure. A basse mer, les palétuviers apparaissent comme plantes surélevées sur racines déchaussées. A la pleine mer, ils sont balancés par la vague ondulante qui ne rompt point leurs attaches élastiques et résistantes. Sur la photographie présente, remarquer le niveau moyen des hautes eaux, très visible.

cinq zones florales principales: $\mathrm{I}^{\circ}$ le centre forestier; $2^{\circ}$ le nord: savane et brousse du haut Nil et de l'Oubanghi; - $3^{\circ}$ le Katanga : forêt, savane et brousse; $-4^{\circ}$ le Kasä̈ : savane et brousse; - $5^{\circ}$ le Bas-Congo d'aspect assez varié : forêt côtière, épaisse forêt du Mayoumbé, savane et brousse en amont de Boma jusqu'au Stanley-Pool.

Dans la zone de la grande forêt on rencontre presque 

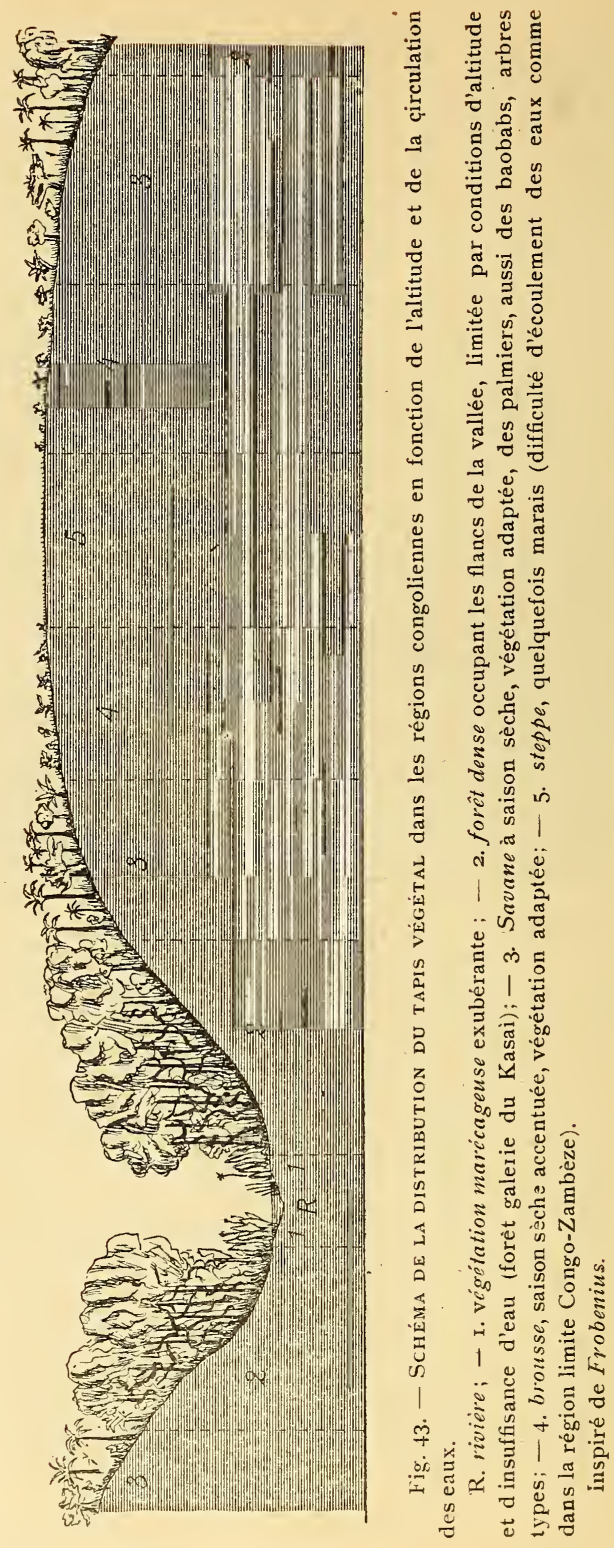
partout le palmier à huile (Elä̈s guineensis), le palmier à vin (Raphia vinifera) et le long des fleuves le dattier sauvage. Ce sont là les essences les plus typiques; l'élaïs de Guinée est commun d'ailleurs sur presque toute l'étendue du

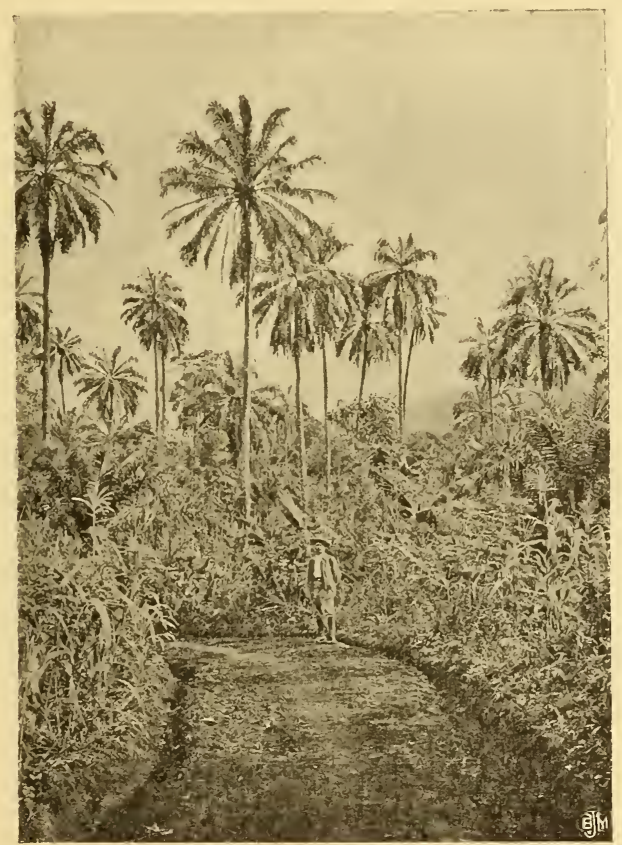

Fig. 44. - Palmiers a huile (Elaïs de Guinée). A leur pied, culture mélangée de mais, de manioc, de banane. Vue d'une plantation dans la colonie allemande du Togo iGuinée) reproduite ici pour montrer le port de l'Elaïs. (D'après Vegetationsbilder de Karsten et Schenck, - G. Fischer à Iéna.)

Congo, il n'est rare que là où l'altitude est grande, par exemple au Katanga. Ajoutons l'acajou, grand arbre qui peut dépasser 60 mètres, le teck, l'acacia, l'arbre à coton, le tamarinier, le kolatier, l'arbre à beurre, le muscadier, les arbres à copal. Outre ces espèces, dans le sous-bois se développe une énorme quantité de fougères, d'orchidées et de 
plantes les plus diverses. Très fréquemment orchidées et autres sont parasites, soit qu'elles absorbent la sève des plantes parasitées, soit qu'elles se contentent de s'en servir comme supports pour parvenir à étaler leur feuillage aux rayons ardents du soleil, par dessus la couronne des grands arbres. Telles sont les lianes, infiniment nombreuses qui, dans

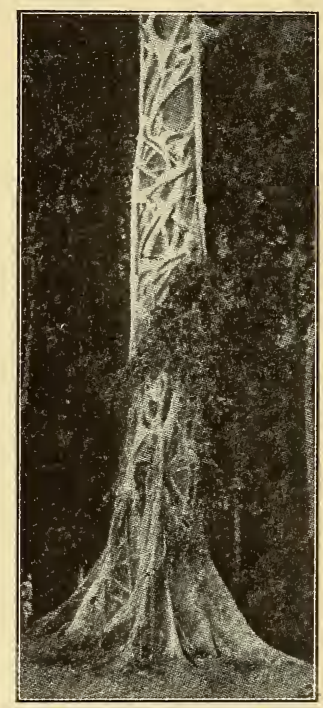

Fig. 45. - Liane ficus enserrant le trone d'un Capoquier (Kapokier) ou Fauxcotonnier dans la forêt de Loukoléla (rive gauche du Congo en aval du confluent de l'Oubanghi). Le Capoquier est un grand arbre (considérer le personnage au pied de l'exemplaire reproduit ici) qui fournit une bourre dont la fibre courte, très douce et abondante est employée déjà en quantité considérable dans la fabrication des coussins, matelas, etc...

un tissu emmêlé, enchevêtrent la forêt équatoriale en un fouillis inextricable que nous a si bien décrit Stanley. Parmi ces lianes, ne citons que les plus communes: les Landolphia, lianes à caoutchouc. La forêt du Katanga - exception faite de la forêt de bambous - se rapproche plus de nos forêts par l'aspect des végétaux et de leur feuillage. 
Dans la savane, les essences forestières ont fortement diminué. Le baobab est apparu, l'acacia se développe, les graminées très vigoureuses envahissent le sol, quelques euphorbes s'y montrent comme témoins du changement du climat. Le palmier est toujours abondant.

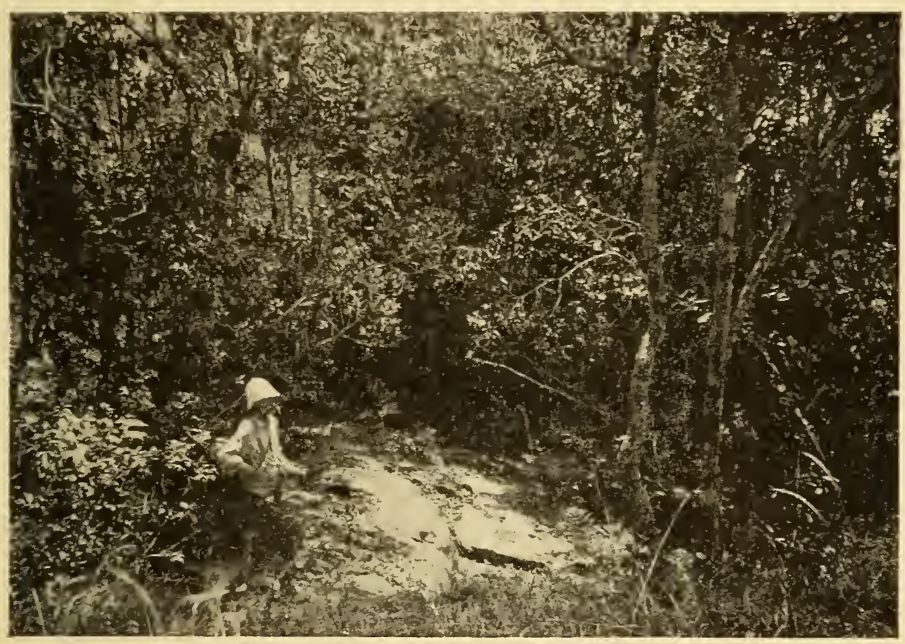

Fig. 46.-LA FORÊT du HaUt-KATANGA, près des sources de la rivière Kouléchi (Congo primaire). Le personnage à l'avant-plan, le carnet de route à la main, est l'explorateur Lemaire qui dirigea plusieurs expéditions scientifiques, notamment celles du Katanga de 1898 à 1900 et du Congo-Nil de 1902 à 1905 , et qui, par ses remarquables travaux d'investigations géographiques, acquit un des premiers rangs parmi les explorateurs modernes.

Cette richesse végétale du bassin entier s'augmente d'une plante qui, avec l'elaïs de Guinée (palmier à huile) suffit à caractériser la végétation congolienne; il s'agit du bananier qui croît sur presque toute l'étendue du bassin. La beauté de son port embellit les sites des villages qu'il entoure; son fruit exquis constitue une des plus riches ressources alimentaires du pays; sa fibre souple et son feuillage géant servent de matière première à l'industrie indigène. 
Quel parti notre ami le nègre a-t-il su tirer de cette abondance végétale? C'est ce que nous verrons tantôt en l'étudiant de près. Nous verrons aussi comment les procédés coloniaux peuvent solliciter cette nature débordante à donner un rendement plus grand encore. Nous nous bornons pour

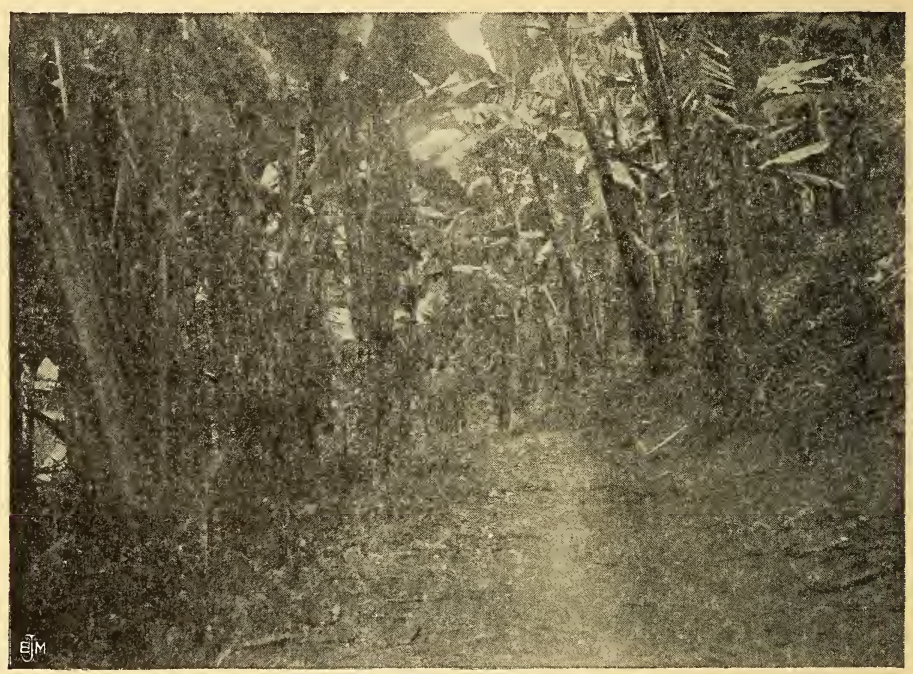

Fig. 47-- Une bananeraie (Photographie communiquée par le Ministère des Colonies).

l'instant à concevoir aussi justement que possible les conditions physiques et biologiques du milieu congolien.

VI. - Les animaux. - Comme bien on pense, la superbe efflorescence de la vie végétale en Congolie y donne nourriture et abri à une vie animale intense: animaux d'eau et de terre, mollusques, vers et arthropodes, poissons, batraciens, :eptiles, oiseaux, mammifères petits et grands.

Cette faune n'est pas particulière à la Congolie, c'est celle de l'A frique équatoriale avec quelques variations sans doute 
qui répondent à la nature du tapis végétal en chaque région $\mathrm{du}$ bassin congolien.

Les mollusques, huîtres et moules marines ou d'eau douce fournissent un tribut considérable à l'alimentation.

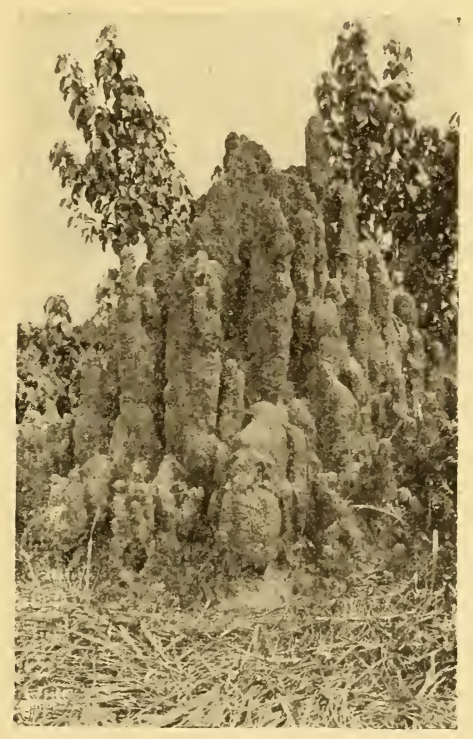

Fig. 48. - UNE TERMITİ́RE. - Les termites, improprement appelés fournis blanches (les termites sont des névroptères, les fourmis des hyménoptères) vivent en sociétés très numbreuses. Comme chez les fourmis, on trouve chez eux des femelles et des mâles pourvus d'ailes et des ouvriers-soldats, sans ailes et bien armés de fortes mandibules. Ils rongent l'intérieur des arbres et des boiseries sans que rien apparaisse au dehors. Sans avoir èté prévu, l'écroulement survient brusquement. Il est quelquefois très difficile de se mettre à l'abri des dégâts des termites. Leurs nids sont de véritables monuments; monticules de terre construits d'argile mélangée de brins d'herbe et de petites fibres végétales, ils atteignent deux, trois et quatre mètres de haut. Leur architecture extérieure et intérieure est compliquée ; la disposition des galeries et des salles répond à un type qui varie peu.

(Phot. communiquée par le Ministère des Colonies.)

Parmi les arthropodes, très nombreux, les crustacés (crabes, crevettes, écrevisses) servent d'aliments. Quant aux autres arthropodes, arachnides, myriapodes et insectes, leur quantité est prodigieuse. Les carabes, cicindèles, hydrophiles, scara- 
bées, cétoines, etc. (coléoptères); les grillons, cancrelàs et sauterelles - celles-ci, dans l'Oubanghi et le sud, émigrent en vols puissants et détruisent les plantations - (orthoptères); les termites improprement appelés fourmis blanches, dangereux pour les constructions en bois, et les libellules (névroptères); les abeilles et fourmis (hyménoptères); les papillons de jour et de nuit (lépidoptères); les punaises des

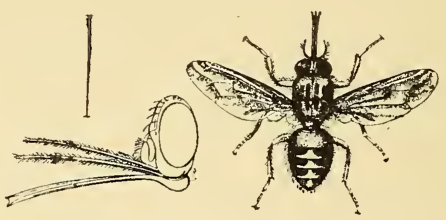

Fig. 49. - La mouche TSETSÉ ou mouche de pirogues, mouche de rivière, mouche à éléphant (glossina palpalis) vit le long des rivières, à l'ombre d'une épaisse végétation. A 500 mètres des cours d'eau, on n'en voit plus. Elle pond une larve unique qui ne supporte pas les rayons solaires. Elle est commune aux abords des endroits où le gros gibier vient boire et se baigner et dans les lieux où les noirs amarrent leurs pirogues. Elle guette dans l'ombre et d'un vol silencieux et rapide fond sur sa proie. Parmi de nombreux insectes mouches et moustiques - agents du transport des trypanosomes, bacilles de la terrible maladie du sommeil qui atteint noirs et blancs, la mouche tsetsé semble être le plus actif. Avec le sang de ses victimes, souvent gros gibier naturellement immunisé ou réfractaire, elle suce les trypanosomes qui se maintiennent dans les petits replis intérieurs de sa trompe, Elle inocule ensuite ces bacilles à de nouvelles victimes hommes ou bêtes. La maladie du sommeil qui a dépeuplé plus d'une région de la colonie est encore incurable. La thérapeutique en est peu avancée, tout au plus peut-elle allonger un peu la vie des malades et adoucir leurs derniers instants. Seuls des moyens préventifs peuvent être actuellement conseillés. Leur efficacité est d'ailleurs problématique parce qu'ils ne peuvent pas être généralisés: les rayons solaires tuant la larve de la glossine (tsetsé), on préconise le déboisement complet aux environs des points d'amarre des embarcations; on préconise aussi la destruction en masse du gros gibier.

bois et cigales (hémiptères); les mouches, tsetsés, moustiques et puces (diptères et aptères); en un mot tous les insectes vivent là en terre promise.

La classe poisson est très richement représentée et constitue pour l'alimentation une ressource inépuisable. Signalons seulement les silurides qui sont les plus nombreux.

Les batraciens, grenouilles, crapauds et salamandres atteignent de fortes dimensions. 
Comme partout où il fait chaud et humide, les reptiles, serpents, lézards, crocodiliens et tortues, sont en abondance.

Les oiseaux pullulent dans toutes les régions. Oiseaux aquatiques, échassiers, colombins, gallinacés, grimpeurs, insectivores et granivores divers ne le cèdent en rien aux prédateurs de jour et de nuit. Plus de la moitié, peut-être les deux tiers des espèces d'oiseaux du globe sont représentés au Congo.

Parmi les mammifères, les mangeurs de végétaux et les mangeurs de chair sont à la fois très nombreux et puissants. Ruminants: antilopes de toutes variétés, de toutes tailles, la girafe, le buffle, le bœuf indigène et le bœuf importé, la chèvre, le mouton. Pachydermes: l'éléphant qui, dans le bassin du Congo rencontre des conditions parfaites de développement, s'est extraordinairement multiplié. On lui a fait une chasse effrayante dans le but de lancer sur le marché du monde une grande quantité d'ivoire. La forêt et la savane sont encore battues à sa recherche, et, justement inquiet au sujet du danger que court ce puissant animal appelé à rendre de très grands services à la colonisation, on réclame des mesures protectrices réglant la chasse. Des décrets en ce sens ont été promulgués. Seront-ils suffisants? On l'ignore encore. L'hippopotame est très nombreux dans tous les cours d'eau, lacs et lagunes. Les sangliers sont abondants. Le rhinocéros est moins répandu (Katanga, Manyéma, Bomou, Nil). Les zèbres vivent en troupeaux dans le Katanga, et aussi dans la savane du Manyéma. L'oryctérope ou cochon fouisseur (sorte de fourmilier) dans le nord et dans le sud, le daman au Nil sont encore à signaler. - Rongeurs : écureuils, porcs-épics, lièvres et surtout rats. - Insectivores: taupes et musaraignes. Chéiroptères: chauves-souris de petite et de grande taille. Carnassiers: le lion peu commun excepté dans le Katanga où il est grand; la panthère et surtout le léopard. Celui-ci 
caractérise la faune carnivore du Congo. La hyène, le chacal, le chien indigène domestiqué, se rencontrent partout. Au Katanga, le lynx. Dans le nord il y beaucoup de mangeurs de reptiles et d'œufs de crocodiles : civette, genette et mangouste. - Les quadrumanes dépassent tous les autres mammifères comme nombre d'espèces et d'individus, particulièrement dans la grande forêt: gorilles, chimpanzés, cynocéphales, cercopithèques et semnopithèques.

Jus ¿iu' à frésent, cette riche faune naturelle n'a servi qu'à satisfaire en partie au besoin d'alimentation et à celui, mal raisonné, du commerce de l'ivoire (ivoire de l'éléphant et de l'hippopotame). Des essais de domestication de l'éléphant et du zèbre ont suffisamment réussi pour prévoir l'importance des services qui seront rendus par ces animaux dans un pays où la mouche tsetsé empêche souvent l'introduction du cheval et du gros bétail. 


\section{L'INDIGĖNE - SON ÉTAT DE CULTURE LA SOCIÉTÉ INDIGÈNE}

I. - Densité Du PEuplement. - Les estimations sur le nombre des habitants du bassin congolais diffèrent entre elles de IO à 40,000,000. Disons provisoirement que I5 à 25,000,000 d'hommes habitent la région du Congo. Cette forte population, en moyenne 9 habitants au kilomètre carré, est très inégalement répartie.

II. - Caractères, Langues et GenRe De vie. -- Pour nous, le mot nègre veut dire : homme à la peau très foncée, noire, brune, quelquefois brun-rougeâtre, au front bombé par le milieu et fuyant, aux cheveux noirs crépus ou laineux, au nez large et épaté, à la bouche lippue, aux pommettes saillantes, à la face prognathe, à la taille assez élevée.

Deux types nègres bien distincts répondent à ces caractères : $\mathrm{I}^{\circ}$ le Nigritien ou nègre du Soudan dont le domaine s'étend du Sénégal et de la Guinée jusqu'aux pays du haut $\mathrm{Nil} ; 2^{\circ}$ le Bantou qui habite l'Afrique centrale et australe et couvre donc presqu'en entier le bassin du Congo. Le Bantou est un peu moins grand que le Nigritien, sa tête moins allongée, son front moins bombé par le milieu est souvent moins fuyant, son prognathisme nioins accentué, son nez plus fort et moins épaté. De plus les langues bantoues sont totalement différentes des langues négritiennes, elles sont caractérisées par l'emploi de préfixes (exemple: Mou-Ntou = la 
tête $=$ l'homme; Ba-Ntou = les hommes). Ces caractères ne sont évidemment pas absolus, les croisements très nombreux entre individus de types différents ont amené la formation

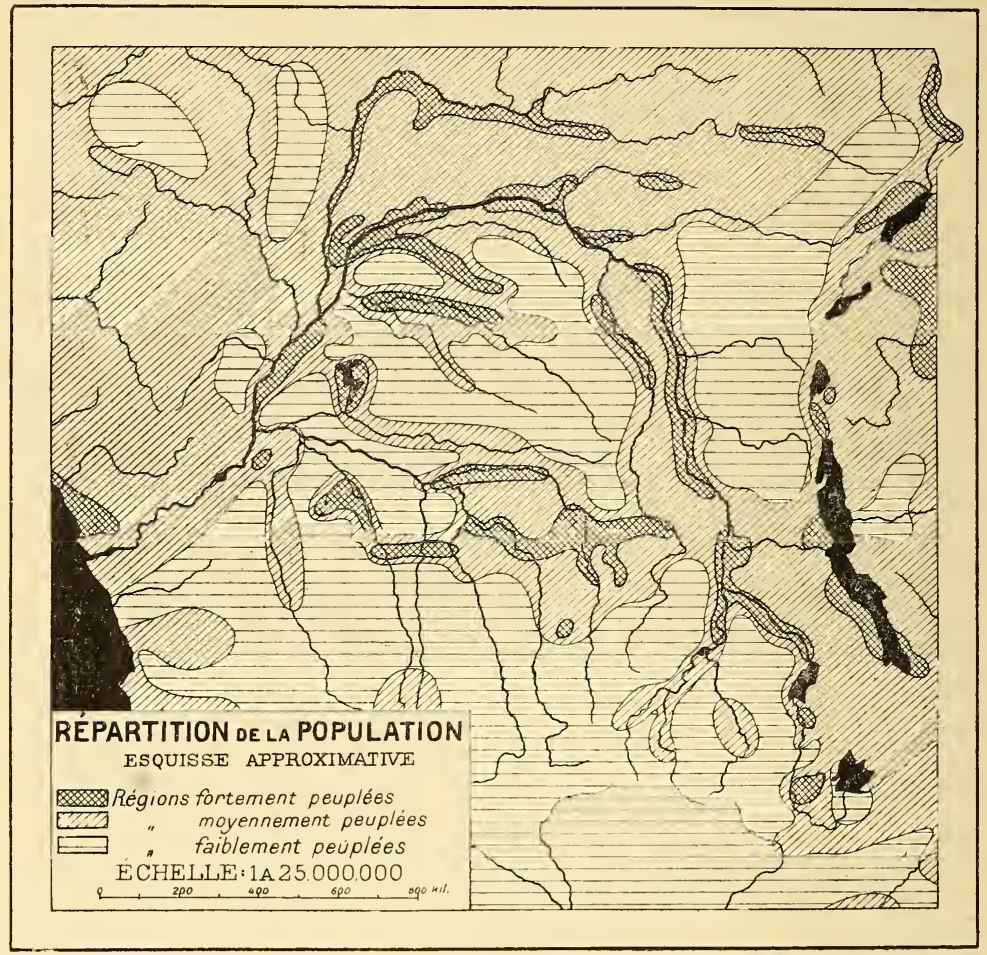

Fig. 50.

(Essai approximatif d'après documents divers.)

d'un grand nombre de types intermédiaires. Les indigènes congoliens sont néanmoins à peu près tous de race bantoue.

Ils parlent des dialectes qui, malgré leurs différences. se rattachent presque tous à la même famille bantoue. Comme les autres nègres, ils n'ont pas d'écriture proprement dite; tout au plus pratiquent-ils l'emploi de quelques signes idéologiques et symboliques. Deux dialectes commerciaux existent: 
$I^{\circ}$ le swahili, moitié bantou moitié arabe de la côte zanzibarite, introduit jadis avec les trafiquants arabes et demeuré la langue commerciale du haut et moyen Congo pendant le temps de l'occupation arabe; $2^{\circ}$ le fiote, de la famille bantoue, influencé de portugais, dans le Bas-Congo.

Le nègre congo peut en général être classé parmi les peu-

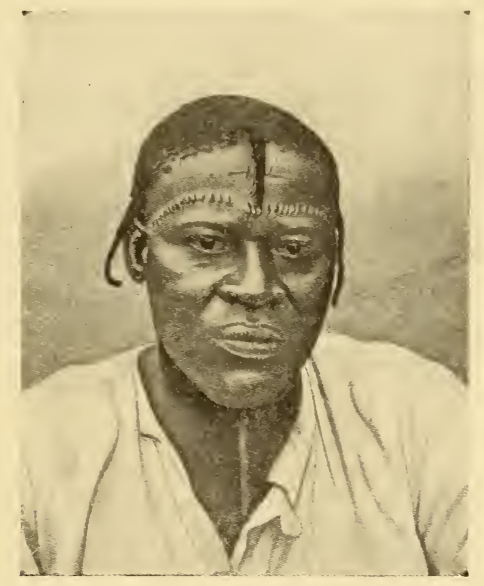

Fig. 51. - Un Bantou.

ples sédentaires. Il pratique beaucoup l'agriculture dans laquelle parfois il excelle; il s'adonne peu à l'élevage en raison de l'existence de la mouche tsetsé. La chèvre, la poule, le cochon, le chien sont les animaux domestiques du nègre. Ajoutons-y le bœuf dans le sud-ouest et le nord-est de la colonie. Les cultures du manioc, de la patate douce, de l'igname, de la banane, de l'arachide dominent dans le centre et l'ouest; celles des céréales: millet, sorgho, éleusine, maïs apportent au noir de l'est le fond de sa nourriture. Le haricot, la citrouille, le riz, la canne à sucre, les fruits du papayer, de l'ananas, du goyavier, du palmier élais fournissent un appoint considé- 
rable à l'ordinaire du nègre. Celui-ci mange aussi de la viande : poule, cochon, gibier produit de la chasse : poursuite avec l'arme de jet flèche ou javelot, traque par le feu, pièges variés. Les rats qui infestent les villages, les lézards très

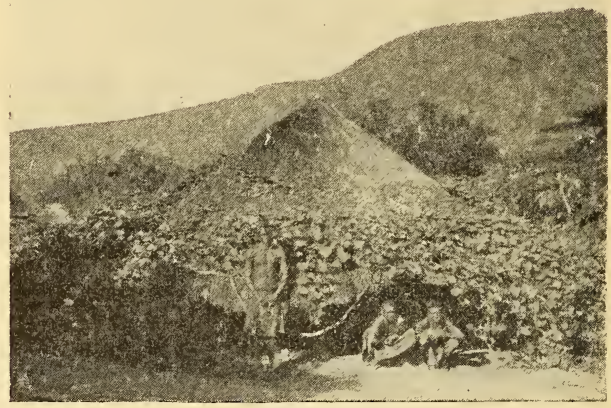

Fig. 52. - Au Katanga. Villagef KA-MAPEMBwB́: 36 huttes disséminées par petits groupes dans des plantations très variées et tres étendues, dont voici l'énumération typique : beaucoup de manioc, maïs, sorgho, loupondo (haut mil à faire le pombé), loukou (petit mil idem), coton (en quantité : on voit les femmes le filer), pommes de terre douces, ignames, pois-cajan, haricot de terre, arachides, sésame (par places, le sésame abandonné à lui-mème est redevenu sauvage et pousse en longues tiges avec de belles fleurs en digitales, mais de maigres et rares gousses), courges, calebasses, ricin (abondant), fougère (médicinier cathartique), tabac, chanvre, hibiscus esculentis, tomates, piment, aubergine amère (de couleur orange-rouge), mioumbou, canavalia (énorme haricot), quelques bananiers, quelques ficus à étoffe, le tephrosa vogelii, qui est une lègumineuse dont le suc sert à stupéfier le poisson. - En ce village, si bien approvisionné, les huttes disparaissent sous une pittoresque garniture de cucurbitacées. (Phot. et texte Lemaire.)

nombreux, les insectes tels que fourmis, sauterelles, qui pullulent, sont très estimés des gourmets noirs. Quelquefois, lorsque l'occasion s'en présente, on mange de l'homme. L'indigène congo boit de l'eau, du vin de palme (malafou), du vin de canne (masanga), de la bière de maïs et de sorgho (pombé), d'éleusine. Comme beaucoup de ses frères supérieurs blancs, il aime s'enivrer. Il a aussi un faible pour les narcotiques : tabac et chanvre, ce dernier dans le Kasaï où l'on en ignore l'emploi comme textile. Les habitants des rives 


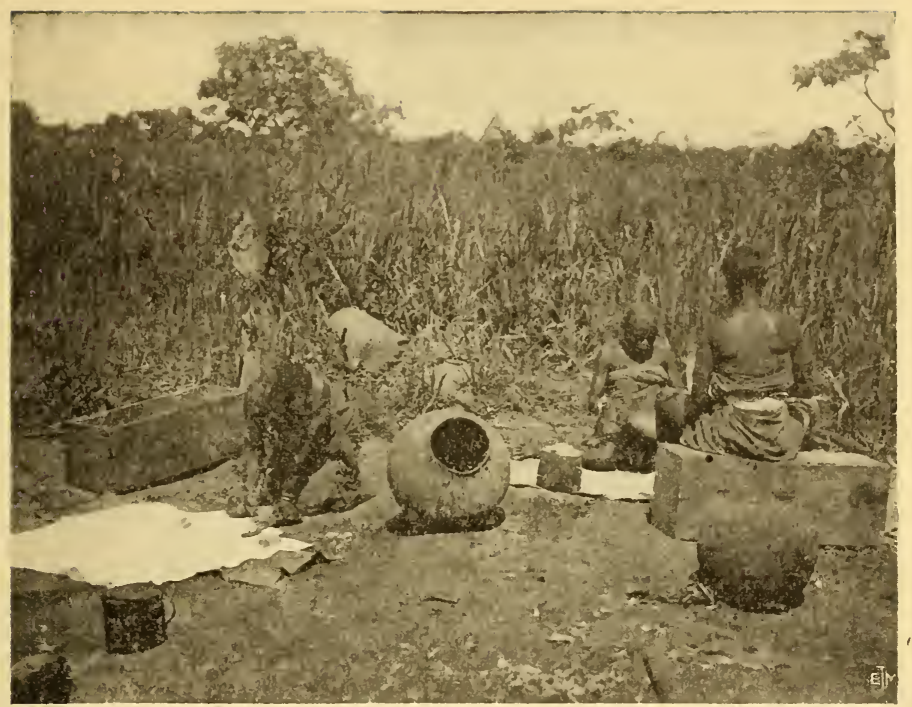

Fig. 53. Femmes préparant la FARINE de mais. Région du nord-est. (Phot. Lemaire.

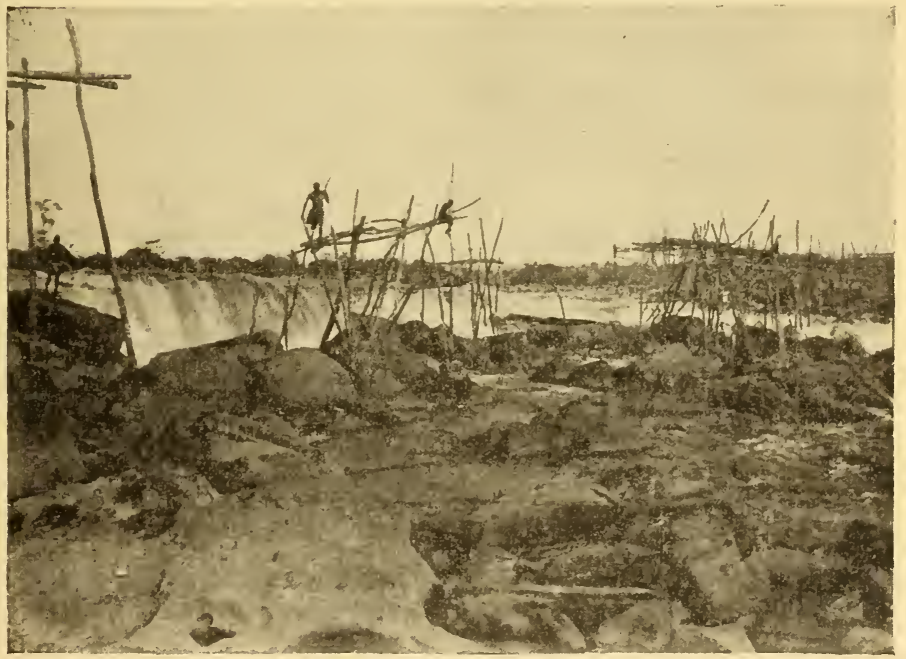

Fig. 54. - Pêcheries dans les rapides du fleuve.

(Phot. communiquée par le Ministère des Colonies.) 


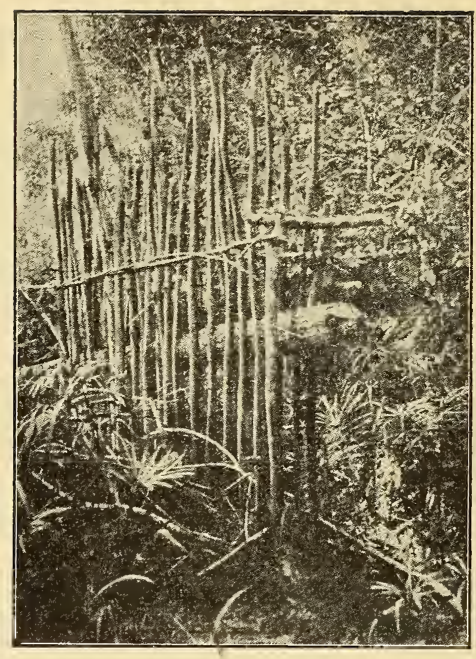

Fig. 55. - * Pik̀ge constítué par un couloir où le gibier - généralement des carnassiers dans ce cas-ci - s'engage pour saisir un appât formé d'un paquet de mulots ou de quelqu'autre petit animal; en saisissant l'appât, l'imprudent fait décliqueter un tronc d'arbre qui l'écrase. 》 (Lemaire.)

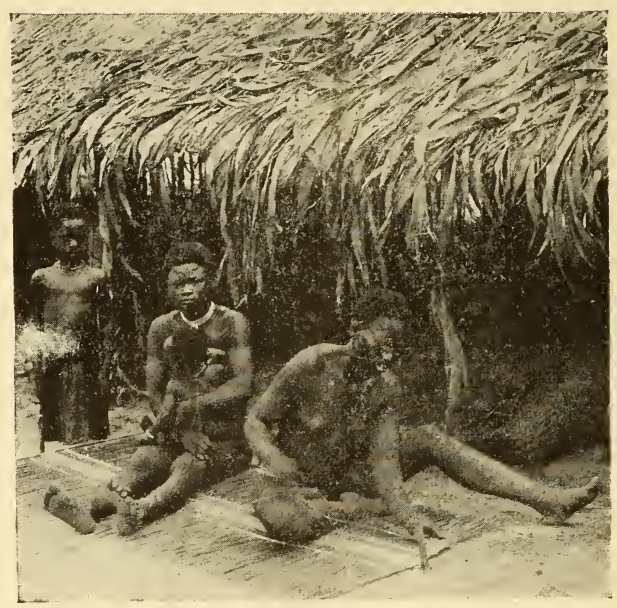

Fig. 56. - Femmes fabriquant des nattes.

(Phot. communiquée par le Ministère des Colonies.) 
des cours d'eau sont pêcheurs. Ils employent les nasses, les harpons, les flèches, les lignes et construisent des barrages

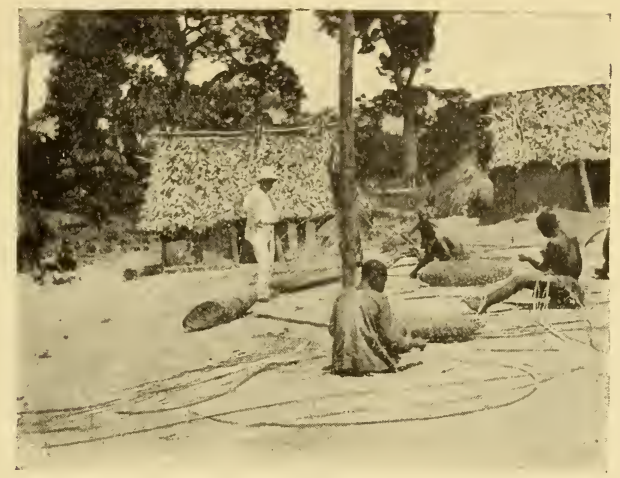

Fig. 57. - Vanniers. Région de I'Ouellé.

(Phot. du Dr Védy, comm. par M. Lemaire.)

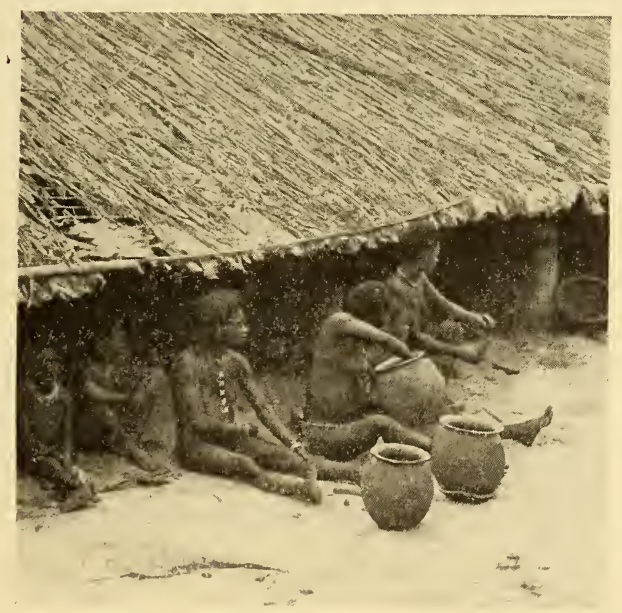

Fig. 58. - Potrères. Région des grands lacs.

(Phot. communiquée par le Ministère des Colonies.)

dans les rapides pour capturer un poisson d'ailleurs abondant. 
Les exigences et les ressources du milieu naturel ont quelquefois fait du nègre un artisan habile. Tels sont tisserands et produisent de jolies étoffes, tels autres sont vanniers et fournissent des ouvrages admirables jusqu'à des sortes de panniers pouvant contenir l'eau, ou bien potiers ou bien

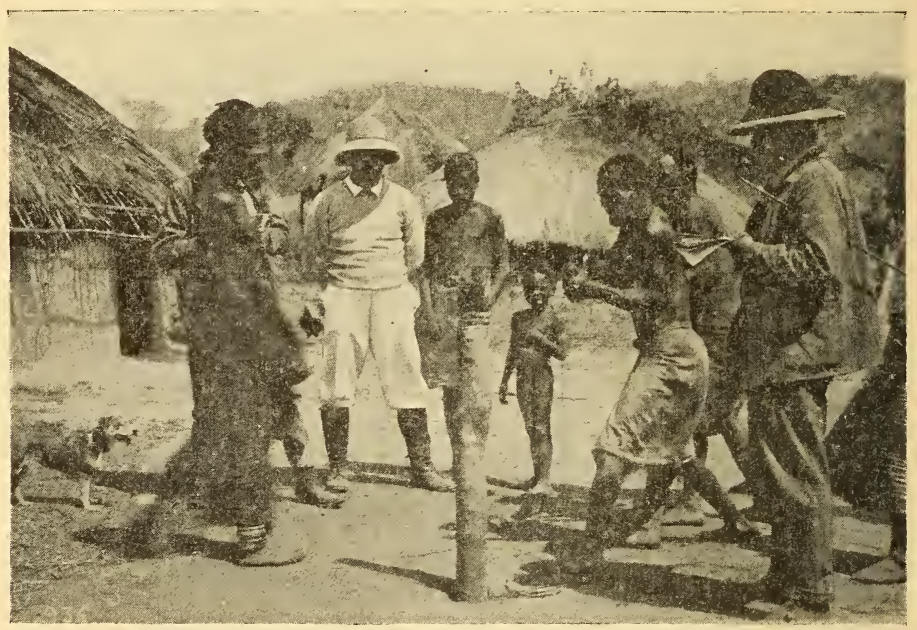

Fig. 59. - Tréfilerie de cuivre. - A la force du bras, l'artisan et son aide étirent le fil au travers d'un trou pratiqué dans une masse métallique fixée sur le poteau. Le trou de filière étant réduit, l'opération est répétée jusqu'au moment où le diamètre du fil de cuivre est jugé convenable. (Phot. et texte Lemaire.)

encore fabricants d'instruments de pêche. Tels autres encore sont sculpteurs sur bois et sur ivoire ou fabricants d'instruments de musique. Tels autres enfin dans les régions à gisements minéraux sont devenus forgerons du fer et du cuivre et poussent cet art très loin, montrant des aptitudes réelles et une patience extrême.

Si certaines tribus sont pacifiques, plutôt sédentaires et cultivent le sol avec amour et ténacité, d'autres sont guerrières. Celles-ci ont le plus longtemps pratiqué et pratiquent encore l'anthropophagie, lorsqu'elles croient à l'impunité. Les 
conflits armés et les véritables guerres de tribu à tribu, de village à village sont fréquents. lls se terminent souvent par

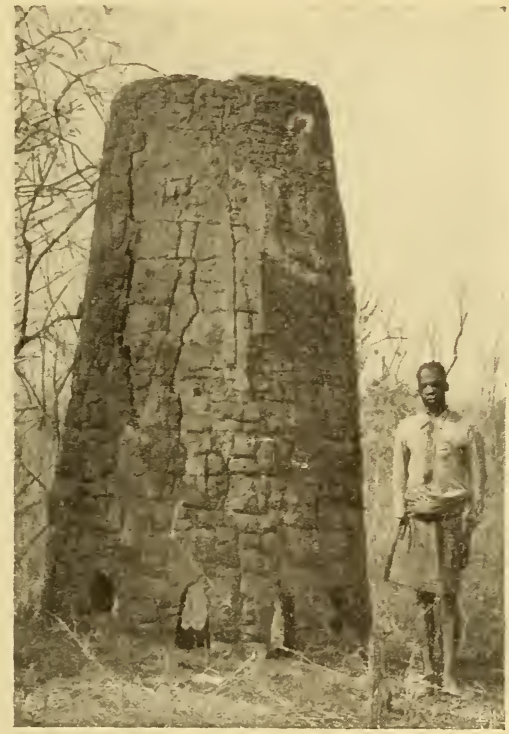

Fig. 60. - Haut-fourneau indigène au Katanga (Lemaire)

la destruction des biens de la tribu vaincue et par des réjouissances cannibales

III. - LE VÊtement. - Si chez notre ami nègre qui n'a pas eu de contact avec l'Européen ou l'Arabe, le vêtement est simple, souvent un pagne élémentaire, quelquefois une cordelette ou rien du tout, néanmoins, il éprouve le besoin de lutter contre le froid de la nuit un peu avant le lever du soleil, en se oignant le corps d'huile ou de beurre végétal, de graisse animale ou même humaine, d'argile ou de bouse de vache (les Dinkas du Nil emploient ainsi la bouse de vache pour se préserver des moustiques). Il pousse assez loin l'art de la 
parure : il se tatoue, portant ainsi la marque indélébile de sa tribu, de son clan et de sa personne; il porte des bijoux de cuivre et d'ivoire, des jambières et brassards en fil de laiton, des bracelets, des colliers de

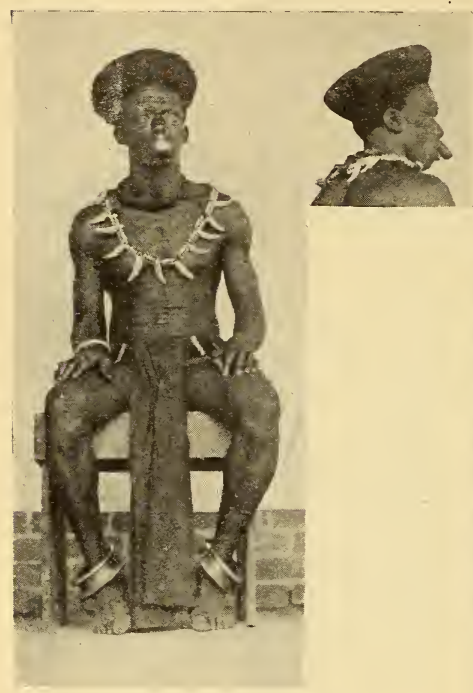

Fig. 6r. - CHEF PARÉ. Remarquer son costume, ses ornements, ses bracelets, son collier fait de dents, son pélélé enchassé dans la lèvre supérieure déformée et allongée en spatule (voir profii), sa coiffure soignée. (Phot. comm par le Ministère des Colonies.)

la lèvre supérieure (pélélé). Il se farde volontiers de terre ocre, brune, rouge et de couleur blanche. Il peut pousser loin la coquetterie cuivre pesant quelquefois plus de io kilogs, divers autres ornements : pendants d'oreille, anneaux de nez, de lèvres, objets enchâssés dans

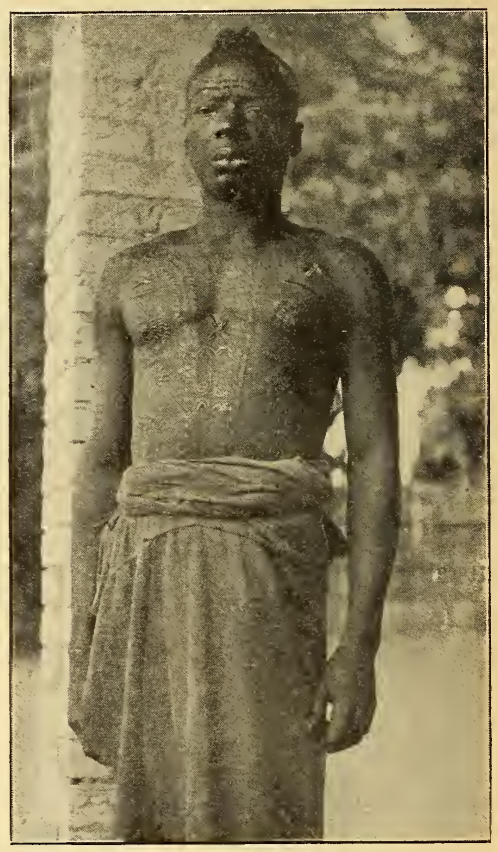

Fig. 62. - Chef tatouÉ. Région de l'Ouellé. (Phot. Dr Védy, comm. par M. Lemaire.) des dents et il les lime en crocs ou autrement. Dans l'arrangement de sa chevelure il révèle un art de perruquier que pourraient lui envier bien de nos posticheurs civilisés. 


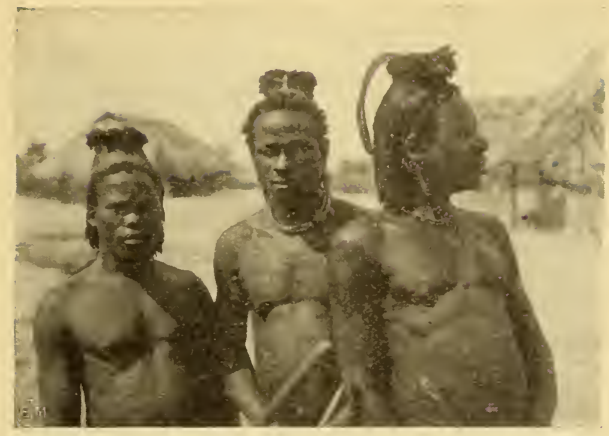

Fig. 63. - Colffures n'hommes. Région orientale (Lemaire).

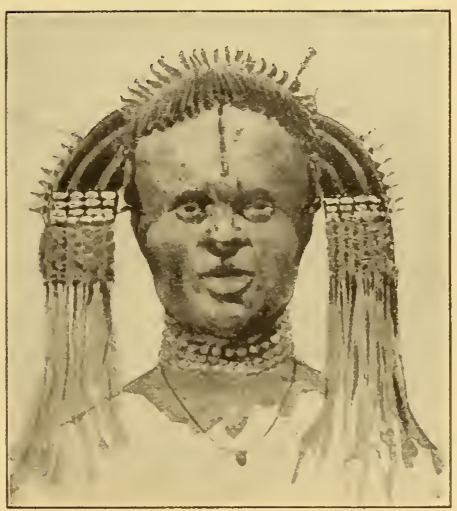

Fig. 64. -- FEMra D'un CHEF du Katanga. Coiffure très soignée et très recherchée ayant demandé plusieurs mois de travail (d'après aquarelle Dardenne, expédition Lemaire). 
IV. - L'HABITATION ET LA FAMILlE. - Le nègre n'accorde à son habitation que l'importance d'un simple abri qu'i]

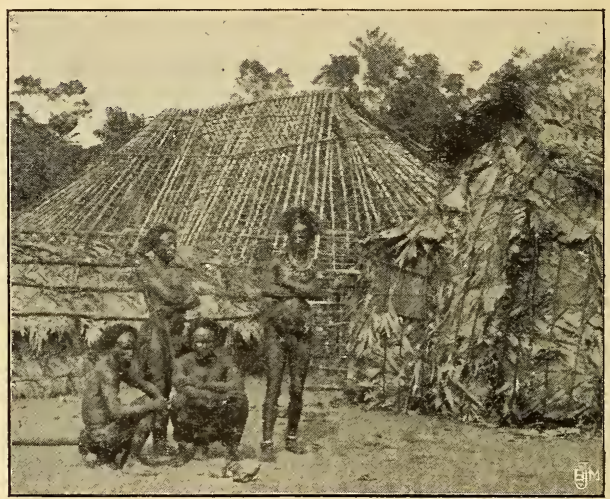

Fig. 65. - Huttes Rectangulaires construites et en construction.

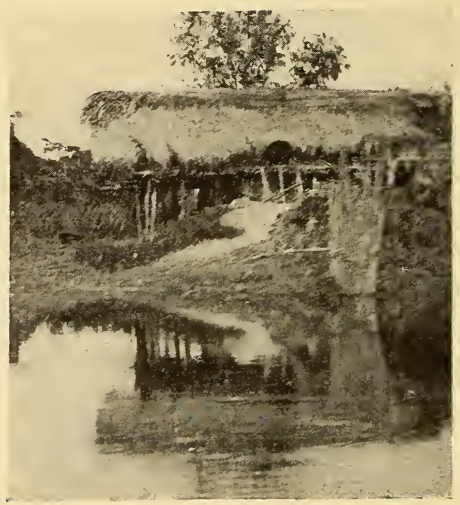

Fig. 66. - Hutte sur Pilotis dans la région du haut Ouellé.

(Phot: Dr Védy, comm. par M. Lemaire.)

sait devoir abandonner à la première alerte. Dans l'ouest, on rencontre surtout l'habitation rectangulaire, dans l'est la hutte ronde. Le nègre qui a subi l'influence arabe, a amélioré sa demeure. 
La famille nègre est d'une tout autre conception que la nôtre. Pour les chefs et les riches, la polygamie est générale. La femme s'achète moyennant bétail, perles ou toute espèce de valeurs d'échange. Si elle est femme libre, fille d'homme libre, elle n'entre pas dans la famille de son mari auquel elle est en somme prêtée moyennant valeurs par sa famille à elle. Ses enfants comme ses biens appartiennent aussi à sa propre famille et y retournent avec elle si son mari meurt.

Ainsi les enfants de femme libre ne sont pas les héritiers

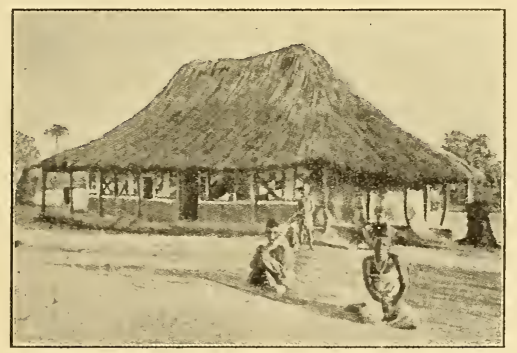

Fig. 67. - MAISON ARABISÉE à Lofoï,_Katanga (Lemaire)

de leur père. Les biens du mari vont au fils aîné de sa sœur aînée. Si la femme n'est pas libre, mais esclave domestique (I), elle est acquise en pleine propriété par le mari pour un prix convenu avec le précédent propriétaire de la femme en question. Les enfants issus d'un tel mariage sont la propriété de leur père, mari de leur mère esclave. Le bénéfice que l'homme trouve dans le mariage est le travail que lui fournit son épouse. Si la femme doit le travail à son mari, celui-ci lui doit l'entretien et la protection. Selon qu'il est bon ou méchant, il peut rendre sa femme et ses enfants heureux ou malheureux. Ia chasse, la pêche, la guerre, voilà souvent le rôle de l'homme. Les soins aux enfants, ce qui

(1) Esclavage domestique : voir plus loin. 
veut dire les laisser croître et jouer, les travaux du ménage et de la culture, voilà le rôle souvent très chargé de la femme. Il y a des exceptions, comme celle des Azandés qui n'achètent plus leurs femmes, chassent, guerroient et cultivent euxmêmes. Nous en dirons quelques mots plus loin.

V. - L'AGgLOMÉRATION. - La population d'un village nègre varie beaucoup, elle peut aller jusqu'à plusieurs milliers, elle est très souvent de deux ou trois cents habitants. Exposés à de multiples dangers, ces villages sont souvent placés sur des hauteurs, ils sont quelquefois construits sur pilotis ; toujours, ils sont peu soignés et très sales.

Le village se dispose ou le long d'un cours d'eau, ou des deux côtés d'une rue centrale ou, fortement aggloméré, il est entouré d'un fossé et d'une palissade de fortification (boma) ou bien encore les huttes se trouvent sans ordre aucun parmi les herbes et les buissons de la brousse.

VI. - L'ORganisation POlitique. et sociale. - Que le village fasse ou non partie d'une force politique plus grande, il a toujours son autonomie locale.

Quelques grands royaumes, véritables organisations féodales, ont pu jadis se constituer : celui de Msiri au Katanga, celui du Mouata-Yamvo, au pays de Lounda. Ces monarchies sont tombées en décadence. Aujourd'hui, le village est le plus souvent lui-même unité politique complètement indépendante et forme avec ses voisins des alliances volontaires cffensives et défensives. I-e chef de grand état, de petile tribu, de simple village est toujours un despote, de pouvoir absolu et héréditaire selon les coutumes lcaales. Il est très rare de constater l'existence d'une camarilla de conseillers pris parmi l'aristocratie qui entoure le chef. Cette aristocratie forme la classe des riches; c'est souvent la famille du chef. Sous cette aristocratie prend place la classe des guerriers, 


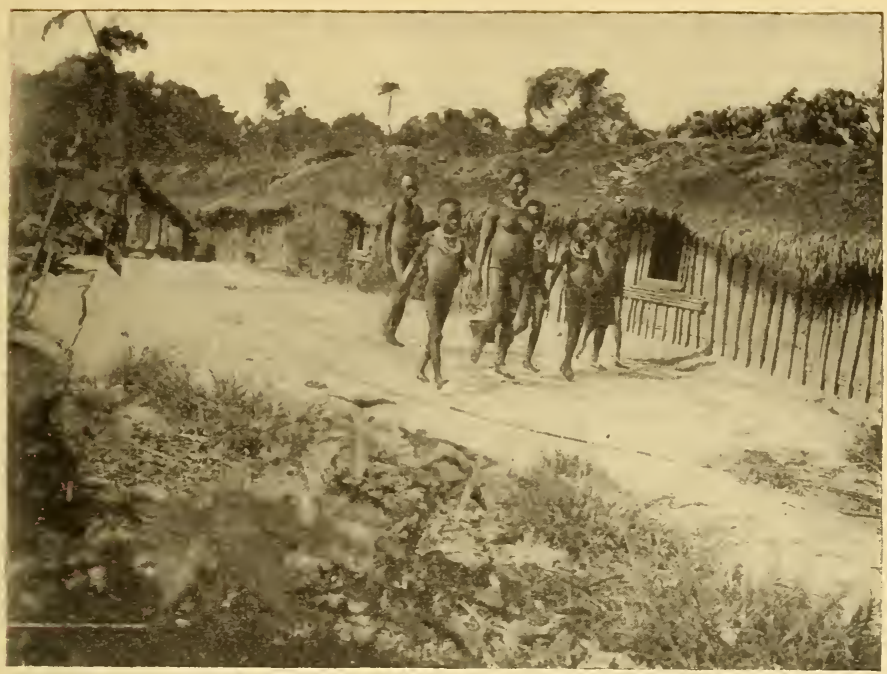

Fig. 68. -- TyPe de Village Rangé, à rue. Région du haut Ouellé. (Phot. Dr védy, comm. par M. Lemaire.)

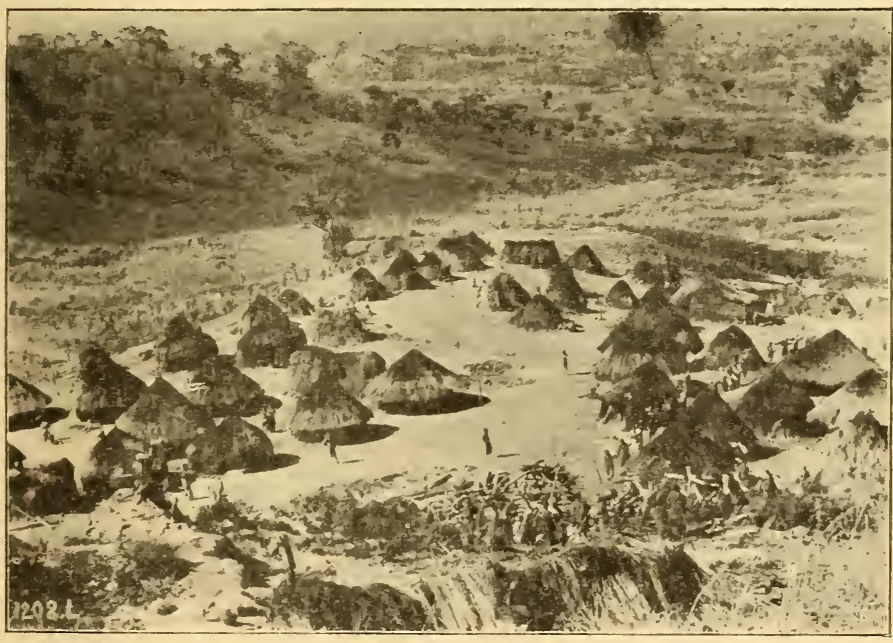

Fig. 69. - Village du Katanga : Tchiamakélé. Espace défriché et battu puur l'emplacement de huttes rondes et disposées çà et là. Une place publique. A l'entour du village on aperçoit un assez grand espace cultivé. Au-delà, la brousse. (Photographie Lemaire.) 
hommes libres, en somme citoyens qui, en assemblée convoquée par le chef et appelée palabre (I), peuvent quelquefois et jusqu'à un certain point limiter l'autorité du chef. Enfin une troisième classe existe dans l'organisation sociale, celle des esclaves domestiques qui sont plutôt domestiques corvéables, vendables et mangeables. Ils s'accomodent, dit-on, relativement bien de cette situation parce qu'en général leur maître use de son autorité absolue en patron familial, bon ou méchant.

"L'esclave domestique au Congo est la propriété quasi absolue de son propriétaire. Celui-ci peut le revendre à qui il veut. A ce point de vue on peut dire que l'esclave domestique est un bétail. Mais c'est un bétail bien soigné et ayant certains droits, du moins dans telle ou telle tribu. Ainsi dans le Bas-Congo (région du chemin de fer des cataractes) un esclave domestique peut se sauver de chez son maître et aller demander protection à un autre maître qu'il choisit librement. Cet autre maître peut accepter, mais alors il doit garder l'esclave réfugié chez lui; i) peut le faire travailler, le punir, etc., mais il ne peut plus s'en séparer, soit en le vendant, soit autrement. Il doit supporter tous les inconvénients comme tous les avantages de son adoption. En réalité l'esclavage dømestique est, règle générale, doux; l'esclave domestique fait partie de la tribu; il s'y attache, finit par s'en croire membre au même titre que les hommes libres; parfois d'ailleurs on le libère en le proclamant homme libre et en lui faisant le tatouage de la tribu par dessus ses tatouages d'origine. Le maitre permet à ses esclaves domestiques de faire famille; et les enfants demeurent sa propriété. Ici encore, l'usage est familial et, sauf circonstances spéciales, ces enfants demeurent dans la tribu. Somme toute, c'est la période féodale du servage, appliquée à l'Afrique païenne " (Lemaire, correspondance).

L'ancienne traite, qui a disparu, était surtout pratiquée par des trafiquants arabes. Elle consistait en expéditions guerrières qui opéraient de terribles razzias et emmenaient la population nègre valide dans le but de la vendre sur les grands marchés de bétail humain. Il est de vastes régions du

(1) Palavra ou palabra, mot portugais signifiant parole. 
centre africain qui ne sont pas encore repeuplées depuis la suppression de cet odieux fléau.

La justice consiste en une application brutale de peines corporelles, souvent de mutilations des organes instruments du forfait ou bien d'amendes, si l'accusé est riche. Ces amendes sont versées au profit du chef. L'accusé n'est jamais autorisé à se défendre.

VII. - Les ÉChANGES, LES MARChÉS, LES ROUTES. - Le nègre est commerçant. C'est là en quelque sorte une de ses

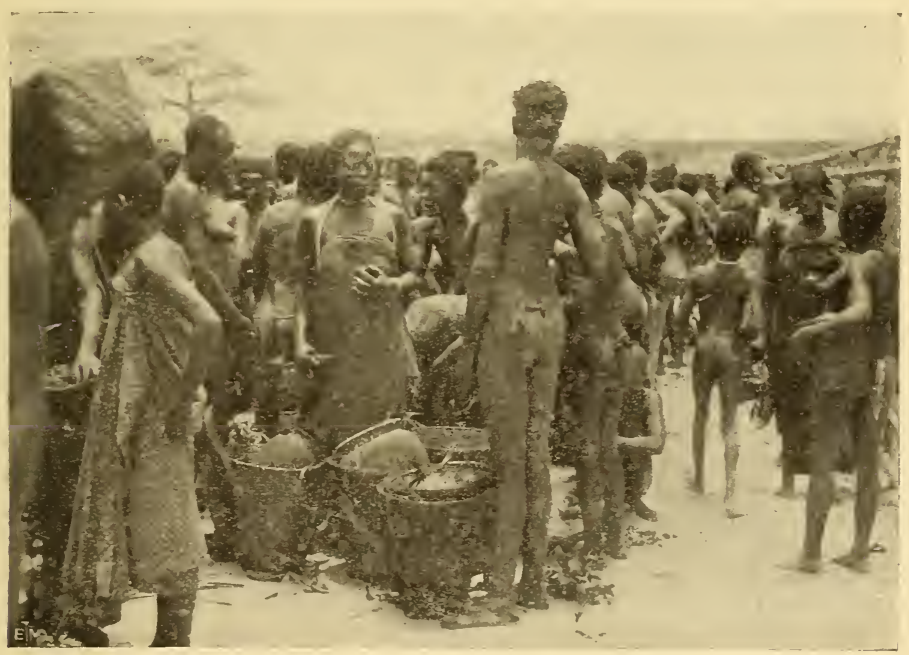

Fig. 70.- ScÈne de MARChÉ à Lokandou sur le Loualaba. C'est au marché qu'on vend qu'on achète, qu'on se rencontre, qu'on apprend les nouvelles, qu'on règle les affaires prirées oı publiques. Il n'est pas de bourgade de quelqu'importance qui n'ait son marché à jour fixé et très bien ordonné. Sur le haut Congo (Loualaba), il y a des marchés à toutes les trois ou quatre lieues, dit Living-tone, et l'on y vient de très loin, car c'est une institution féminine non moins entrée dans les mœurs que chez nous de courir les boutiques.

(Phot. comm. par le Misistère des Colonies.)

propriétés spécifiques. Il possède cet art complexe de présenter et de faire valoir ou de déprécier la marchandise selon 
qu'il est vendeur ou acheteur. Il pratique encore l'échange en nature, le simple troc, mais le plus souvent a établi une véritable monnaie. Les étalons d'échange qui ont cours sur les marchés sont les étoffes, les perles, les coquillages (en voie de désuétude), les pointes d'ivoire, les lingots et les bâtonnets (mitakos) de cuivre ou de laiton, dans certaines régions, le

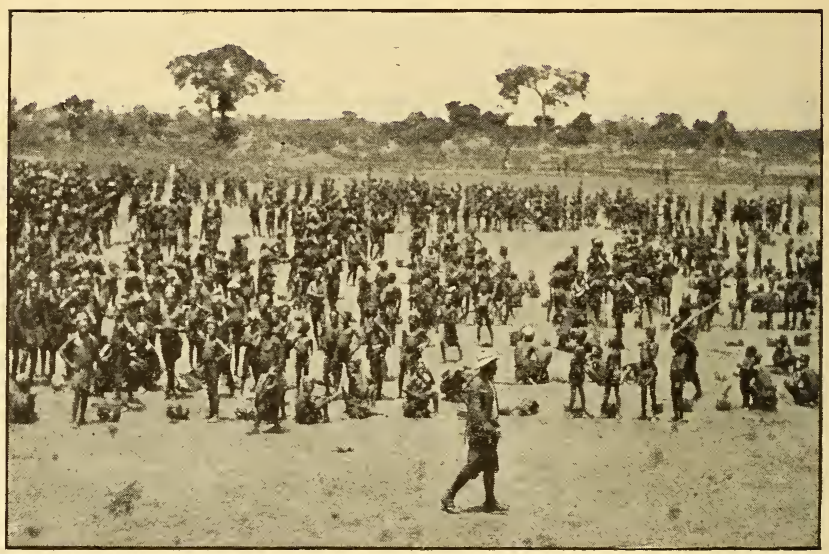

Fig. 7I. - Le marché des Mangbettous a Nyangara.

petit fragment de caoutchouc. Le domestique, l'esclave indigène est lui-même étalon d'échange.

Le marché public est d'importance vitale pour le nègre.

Les routes indigènes parcourues de tout temps par les noirs sont des sentiers pratiqués à travers la savane ou la brousse. Etroits, de largeur d'hommes, ils se déroulent sur de très longs espaces, à travers plaines, plateaux et montagnes, suivant rarement le fond des vallées, souvent à mi-côte d'un versant ou même, lorsque la nature tabulaire du pays le permet, coïncidant avec la ligne de faîte de deux bassins de ruissellement. On doit de très curieuses observations à l'explorateur Lemaire au sujet des commerçants et des colporteurs 
noirs trafiquant très activement de la côte occidentale portugaise à la Zambézie et au Katanga. Voyageant en grand nombre, quelquefois à plus de $\mathrm{I}, \mathrm{nOO}$, ces Wamboundous tel est le nom de ces négociants indigènes — ont tracé d'importantes routes de portage. Au sud du Katanga il en est notamment une, dite le grand chemin des Wamboundous, suivant la ligne de faîte Congo-Zambèze et faite d'une dizaine de sentiers parallèles. Les fortes colonnes de colporteurs la parcourent sur une dizaine d'hommes de front. Nous signalons spécialement ces Wamboundous parce qu'ils sont les plus curieux des commerçants nègres et parce que tous les pays qu'ils draînent, très pacifiquement d'ailleurs quoique marchands d'esclaves, pays riches, prospères et hospitaliers, nous offrent une preuve de plus de la nécessité de pénétrer la colonie par l'éducation lente et progressive des peuples noirs. "Les Wamboundous, dit Lemaire (I), sont des colporteurs ; parmi eux, les uns travaillent pour leur propre compte, d'autres se groupent; si leurs moyens le leur permettent, ils arrivent de l'ouest accompagnés de porteurs libres; pour le retour vers l'ouest, la campagne d'échanges terminée, les Wamboundous ont comme porteurs supplémentaires les esclaves achetés au cours de leurs opérations commerciales. Il importe de remarquer que les Wamboundous $\mathrm{du}$ moins en règle générale — ne se livrent aucunement à des razzias ; ils ne capturent pas eux-mêmes des indigènes à emmener en esclavage.

"Ils achètent des esclaves comme ils achètent tout autre produit commerçable: caoutchouc, ivoire, cire, peaux, etc., etc. Pour ces achats, ils apportent à l'intérieur tout ce que l'on peut trouver à la côte comme articles européens, l'alcool excepté. Somme toute, ces gens ont transporté au loin dans

(1) Lemare, Les Wamboundous, les colporteurs noirs entre litlantique et la Ka-Tanga. - Revue de géographie. 1902. 
l'intérieur le commerce apporté et instauré par les blancs à la côte, depuis cinq siècles. Ils ne font, au Centre Afrique, que ce qu'ils ont appris des Européens à la côte. On me semble donc très mal venu de les considérer comme des bandits sur lesquels il faut tomber à bras raccourcis. C'est à la fois injuste et maladroit.

" Injuste, puisque ces gens, je vous le répète, ne font qu'imiter ce que leur ont appris nos prédécesseurs en Afrique; injuste encore puisque ces gens ne funt que le commerce librement accepté par les indigènes (les capteurs d'esclaves sont les chefs indigènes et c'est sur eux qu'il faut agir); injuste parce que si les Wamboundous viennent acheter beaucoup d'hommes dans le Centre Afrique, c'est pour les envoyer vers l'océan Atlantique où ces hommes deviennent les travailleurs des Européens ; en particulier les "contractés " envoyés à San-Thomé et à l'île du Prince, sont souvent de provenance wamboundou.

" Maladroit aussi est-il de tomber à main armée sur les Wamboundous ; j'ai dit combien le pays parcouru par eux était peuplé et riche ; combien les populations étaient accueillantes et confiantes; comment les villages étaient largement ouverts au passant honnête. L'action des commerçants wamboundous n'a donc pas eu que des conséquences désastreuses, bien au contraire ; ils ne commercent pas que le bois d'ébène, mais aussi beaucoup de caoutchouc et de cire ; ils apportent aux indigènes des quantités de produits manufacturés et leur venue annuelle est nécessaire à la vie de ces régions.

"Si, par un moyen quelconque, on parvenait à refouler d'un seul coup les Wamboundous, on provoquerait dans les pays habitués à les voir, un mécontentement général et profond qui se manifesterait peut-être par des agressions sérieuses contre les nouveaux occupants du pays.

"Cependant l'Européen est aujourd'hui en train d'occuper lui-même par ses postes gouvernementaux et par ses factoreries 
de commerce, le pays parcouru séculairement par les Wamboundous. Que va donner cette concurrence?

"Pour moi, il me paraît nettement que la première action gouvernementale doit être l'établissement d'un certain nombre de stations frontières, commandées par des Européens de choix, dépendant d'un commandement unique ; ce commandement unique devra être pénétré de l'idée que les Wamboundous sont de simples commerçants, sans plus, et que, pour les battre sur leur terrain, ce n'est pas aux fusils qu'il faudra avoir recours, mais à la concurrence commerciale; les Wamboundous, qui sont rompus aux mœurs des Européens de la côte, ne feront aucune difficulté à se soumettre aux règles de douane; pour que cesse leur commerce d'hommes, il suffira d'agir sur les chefs négriers du pays. ") (I).

\section{VIII. - LES CROYANCES ET LES ARTS. - Le nègre n'expli-} que pas les forces de la nature, il en est le sujet et croit aux esprits du bien et du mal qu'il incarne dans les êtres animaux et végétaux et dans les objets les plus divers. Il tâche de se les rendre propices par des offrandes et des sacrifices. Tout nègre a ses amulettes et ses talismans, se façonne des fétiches.

Toute tribu, tout peuple a ses dieux, ses fétiches. Le chef impose quelquefois un culte à ses sujets pour mieux les dominer, par exemple celui du chanvre chez les Baloubas du bassin du Kasaï, en remplacement des anciens fétiches qui furent brûlés en public.

Les féticheurs disposent en général d'un très grand pouvoir ; ils sont à la fois prêtres, sorciers, médecins et conseillers du chef. Ils rendent la justice si le chef n'est le justicier.

Le nègre aime la musique. En général, il est compositeur assez monotone. Chez certaines tribus du Kwango et de l'Ouellé, chez les Azandés dont nous parlerons plus loin, on

(1) C'est nous qui avons souligné certains passages. 
entend de belle musique touchante ou guerrière, mélodieuse et inspirée. On rencontre des orchestres complets composés de tambours, tam-tams, xylophones, grelots, gongs, castagnettes, harpes, mandolines, flûtes, etc... On signale au loin l'arrivée des solistes réputés.

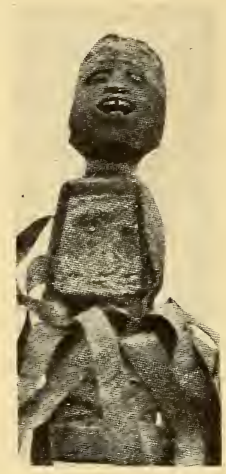

Fig. 72. - Un FÉtrCHE. - Les fétiches ne sont pas des idoles. Leur puissance leur a été attribuée par l'indigène lui-même. lls symbolisent les esprits favorables ou nocifs et perfides. Le nègre les flatte et leur offre des cadeaux ou les effraie et les frappe pour les fléchir Fétiches vivants ou fétiches figures d'êtres animés ou de choses.

La trombe d'ivoire est très en honneur et, avec le gros tamtam, sert aux communications lointaines de village à village.

De l'art musical, chant ou instrument, le nègre ne sépare jamais l'art de la danse qu'il adore et qu'il pratique avec une adresse et une fougue qui ne sont guère appréciées de l'Européen.

Par dessus tout, le nègre aime les contes. Il est lui-même bavard et s'écoute volontiers parler. Le soir les hommes se réunissent sur la place du village ménagée à cet effet, y boivent le pombé et conversent avec animation, insouciance et gaîté jusque fort avant dans la nuit. 


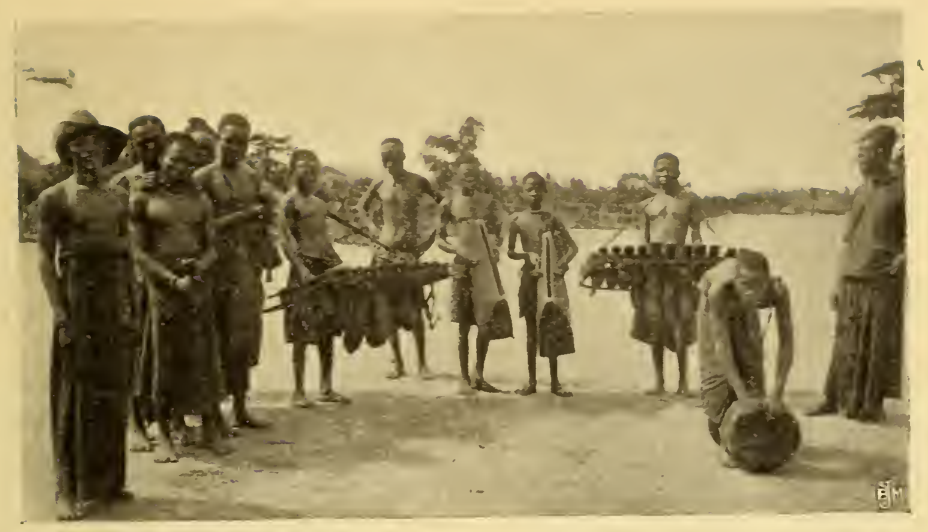

Fig. 73. - UN ORCHESTRE.

(Phot. comm. par le Ministère des Colonies.)

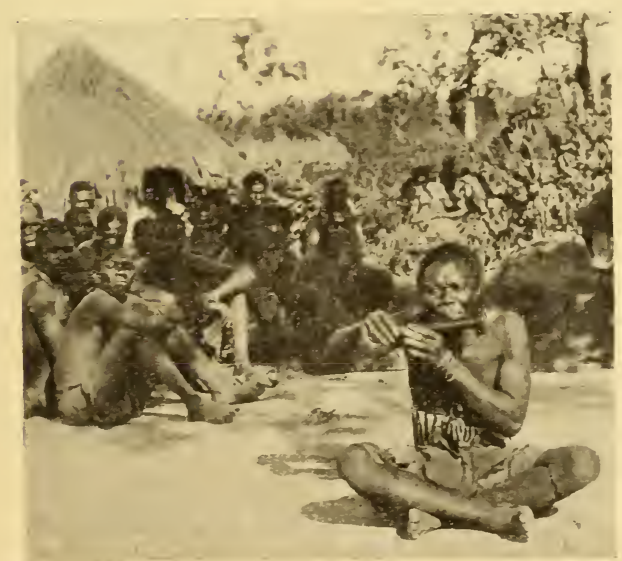

Fig.7f.-UN soliste. \&.e musicien a l'air vivement intelligent, met vraiment du sentiment dans l'exécution des divers morceaux, dont il est naturellement le compositeur; il a plaisir à charmer son auditoire; tous nos gens sont groupés autour de lui. Pendant une demiheure il nous amuse fort. Finalement la conversation suivante s'engage : - « D'où vientta gouina-ia-molomo (c'est le nom de la flute)? - Je la fis moi-mème. - Alors tu pourrais en faire une autre? -- Oui, bien certainement. - Je voudrais acheter celle-ci. , L'homme semble se consulter, puis, sans un mot, il me tend l'objet convoité par le collectionneur blanc; je lui remets deux brasses d'étoffe, ce qui semble un roỵal paiement, à en juger par l'expression que prend sa bonne frimousse. " (Phot. et texte Lemaire.) 
IX. - LES TRIBUS. - Nous n'avons pas besoin de signaler ici les divers peuples qui constituent l'ensemble de la population congolienne. Nous indiquerons seulement les tribus les plus marquantes appelées, semble-t-il, à jouer un rôle important dans l'évolution sociale des Congos et celles qui présentent un intérêt particulier comme, par exemple, les nains de la forêt. Ne perdons pas l'occasion d'observer l'influence du milieu naturel sur le degré de culture et de civilisation de nos amis. Dans la région côtière et sur le basfleuve, les indigènes ont subi, depuis quatre cents ans, l'influence européenne. Dans la région forestière, l'agriculture s'est forcément moins développée que dans la savane; les communications y étant difficiles, les groupements indigènes sont restés plus isolés les uns des autres, le caractère plus farouche, le degré de civilisation moins élevé ; l'anthropophagie s'y est aussi maintenue davantage. Dans la savane et dans la brousse, les conditions de climat et de communications sont plus favorables; l'agriculture, l'industrie et le commerce se sont mieux développés; les populations sont moins cruelles et plus pacifiques, quoique de grandes et longues guerres, conduites par des chefs puissants, aient tourmenté de vastes territoires.

Une classification des peuples congos, basée sur cette notion de l'influence du milieu naturel sur l'homme, apparaît ici comme seule nécessaire. C'est là d'ailleurs une classification purement géographique.

$I^{\circ}$ Bantous-Congos de la côte et du bas-Congo. - Sont assez mêlés, ont fortement subi l'influence européenne depuis quatre siècles. Vigoureux et intelligents, superstitieux comme tous les nègres. Parmi eux les Bakongos fournissent beaucoup de travailleurs à l'État et ont donc en partie abandonné l'agriculture et la pêche; les Batékés, très noirs, peu tatoués, coquets, commerçants piroguiers, potiers, agriculteurs. 
$2^{\circ}$ Bantous-Congos du centre forestier. - Restés plus indépendants les uns des autres et sans contact aucun avec le dehors. Brillantes qualités physiques et intellectuelles. Vices pénibles ancrés profondément : cruauté et anthropophagie. Les Bangalas, au tatouage et à la coiffure spécifiques. Sont beaux, grands, intelligents. Leurs villages, composés de huttes rectangulaires, sont vastes et nombreux. Artisans du fer. Piro-

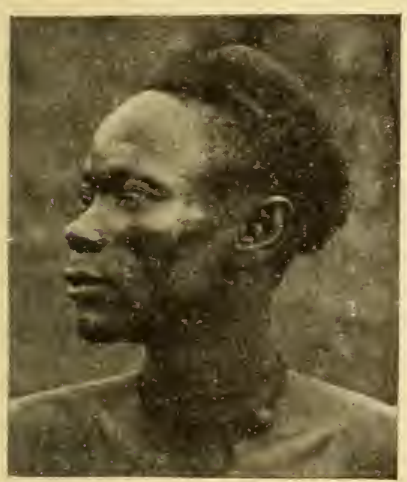

Fig. 75. - HoMME BatéKÉ.

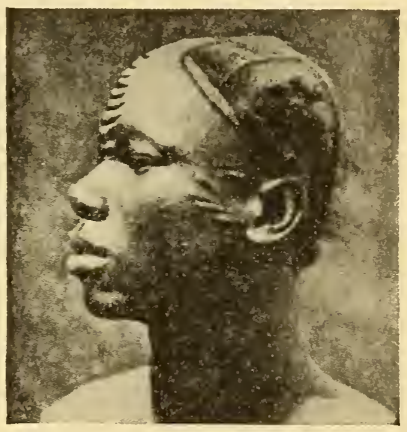

Fig. 76. - Jeune homme Bangala. (D'après le Mouvement géographique.

guiers célèbres (grande pirogue basoko). Guerriers de carrière. Anthropophages. - Les Mongos, forgerons habiles, commerçants, ont sourent été attaqués et mangés par les Bangalas. Les Oubanghis, dans un pays moins boisé, ont subi l'influence du nord. Agriculteurs sédentaires (manioc, canne à sucre, arachide, banane, patate, féverolle) comme les Gobous, ou bien chasseurs-pêcheurs exclusivement nomades, comme les Banziris, ou bien encore mi-chasseurs, mi-cultivateurs, vivant dans de grands villages comme les Bongos (luttes souvent sanglantes entre les sédentaires et les nomades brigands).

$3^{\circ}$ Bantous-Congos de la savane et de la brousse ou du sud et du sud-est. - Plus grande unité de type, de langue et. de mœurs. Les Baloundas ou Loundas (ancien royaume du 
Mouata-Yamvo, du Kasai au Loualaba). Grands, de teint clair, à la bouche lippue. Très commerçants, en général pas travailleurs; exceptions très laborieuses et industrieuses. - Les Balou-

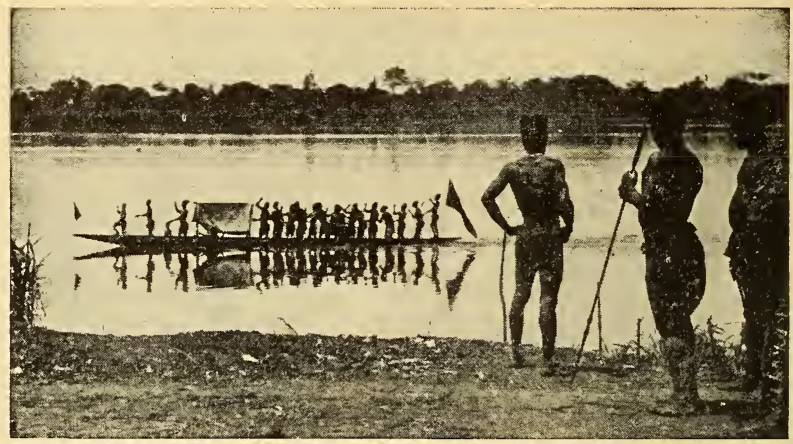

Fig. 77. - Pirogue bangala sur le Congo.

bas, au nord des Balounda;, de la Louloua au Tanganika. Paisibles, d'abord facile, très agriculteurs (irrigations; manioc,

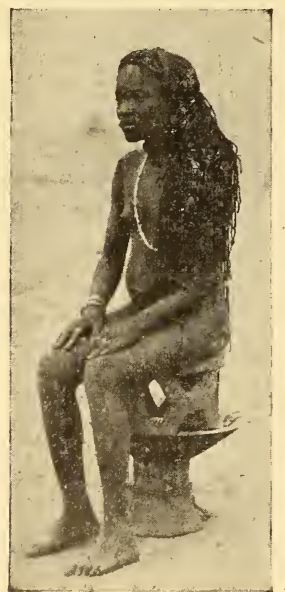
maïs, millet, arachides), commerçants, artisans du fer. Villages populeux jusqu'à 3,000, 4,000 habitants, même plus. - Les Bakoubas, du Sankourou inférieur et du Kasaï, de taille moyenne et de traits fins, sont d'un type beau et pur. Agriculteurs pacifiques, aimables. Artisans du fer, du tissage, de la poterie, de la vannerie, sculpteurs, commerçants honnêtes. Pagayeurs habiles. Non anthropophages. Vivent en gros villages. Organisation féodale (chef roi et chefs vassaux). - Les Batetelas, voisins des Bakoubas, remarquables aux points de Fig. 78.- Jrune fille odbaxam. vue physique et intellectue?. (1 hot. comm. par le Ministère des Colonies.) 


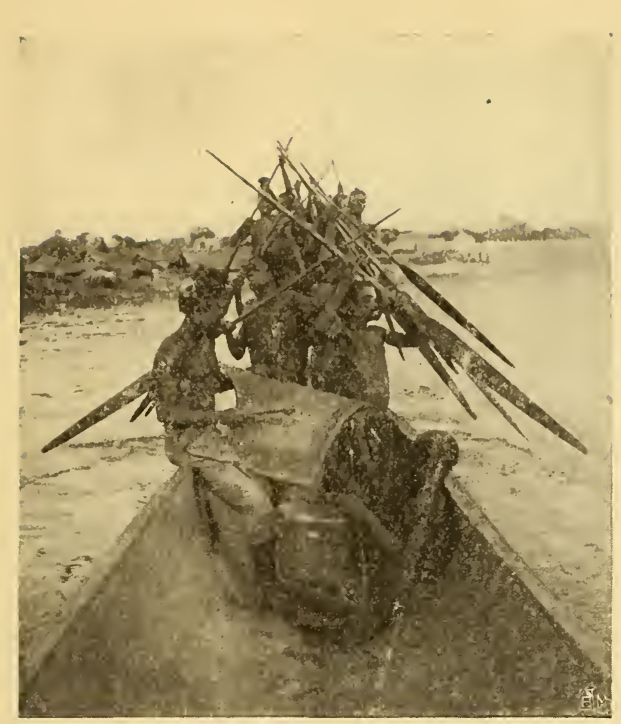

Fig. 79. - Piroguiers sur le haut Congo (Loualaba). lls pagayent en chantant au rythme d'une musique cadencée qui harmonise les mouvements de tous et règle ainsi la marche de l'embarcation. Les indigènes représentés n'appartiennent probablement à aucune des peuplades citées dans notre aperçu. (Phot. comm. par le Ministère des Colonies.)

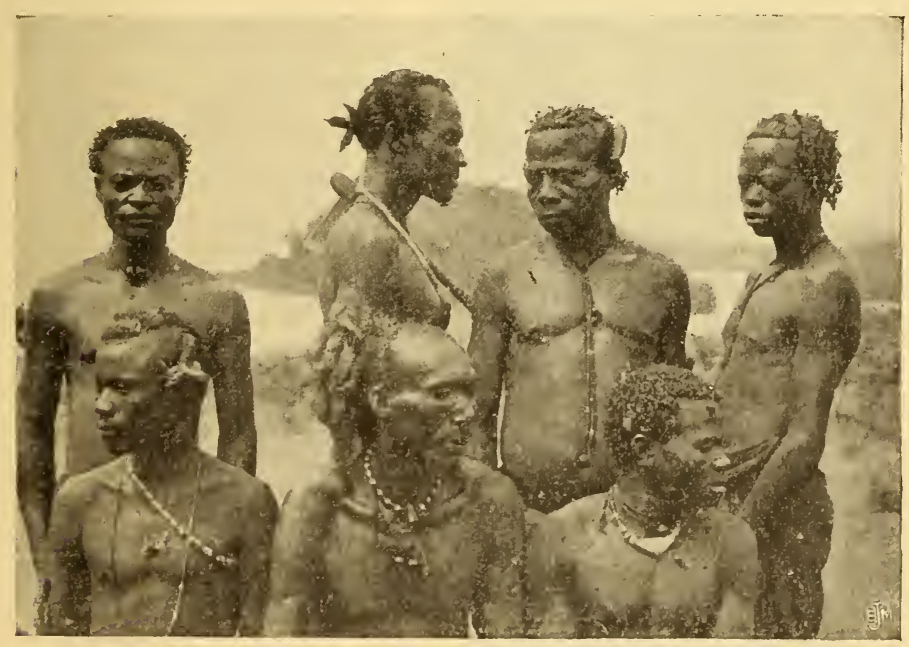

Fig. 8c. - INdigk̀ies de L'Ourova (lac Moéro). Affinités avec les Baloubas. (Photographie Lemaire.) 


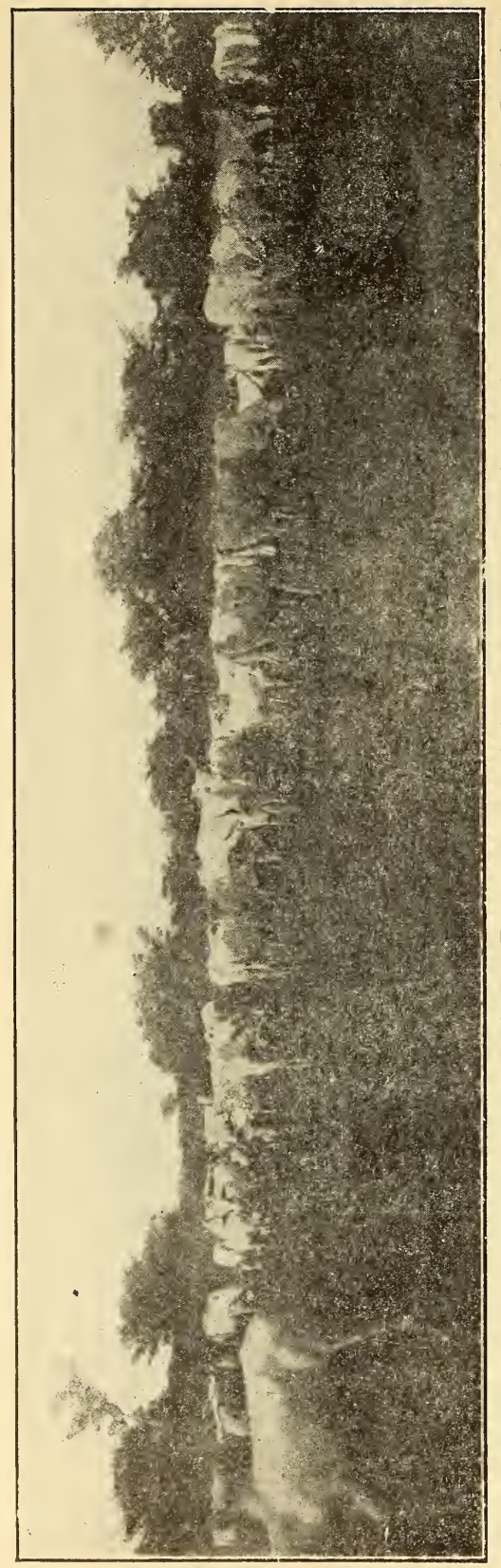

$4^{\circ}$ Les Nilotes. - Dans l'extrême nord-est de la colonie, répandues sur la région faîtière des bassins du Congo et du Nil, s'étendant sur toute l'enclave de Lado et sur une grande partie du Soudan oriental, vivent des populations nigritiennes qui ont entre elles des affinités de langue. Ce sont les Nilotes, nègres qui n'ont que fort peu de contact avec les Bantous et chez lesquels, par suite d'influences diverses, notamment arabes, une division curieuse s'est établie : les uns, comme les Baris, établis sur le Bahr-el-Djebel, sont sédentaires, agriculteurs (sorgho, millet, manioc, patate douce, haricot, banane, tabac,) possèdent des troupeaux assez nombreux, sont artisans et vivent en groupes denses; d'autres, comme les Alouris, sur les rives du lac Albert, sont exclusivement nomades, pêcheurs, commerçants, à tendances très pacifiques; d'autres, enfin, nomades aussi, sont chasseurs de la brousse et guerriers.

$$
5^{\circ} \text { Les Négrilles. - Dans }
$$


la forêt dense, éparpillés et fuyant les Bantous, vivent des indigènes de très petite taille, de $\mathrm{I}, 35$ mètre à $\mathrm{I}, 55$. Teint relativement clair et rougeâtre; cheveux crépus et courts, noirrougeâtre; tête grosse; base du nez large; pommettes faibles: maxillaires très hauts; bouche non lippue à aspect simiesque; tour du thorax faible; ventre gros; membres grêles; démarche sautillante. Exclusivement nomades chasseurs; ont des huttes à terre ou perchent dans les arbres sous des abris. Ils échangent leurs produits de chasse contre les produits agricoles de leurs voisins : ils déposent la viande en un endroit habituel où les cultivateurs voisins viennent la chercher et la remplacer par des bananes par exemple. Les plus typiques sont ceux de l'Arouwimi, que Stanley vit pour la première fois : Akka ou Tiki-Tiki, vivant aussi au pays des Azandés. Les Watwas ou Batouas dans la forêt de la rive gauche et des affluents de gauche du Congo moyen (Lomami, Tchouapa) sont moins purs. Certains négrilles sont anthropophages. Ils fuient l'étranger blanc ou noir et par conséquent sont peu étudiés.

$6^{\circ}$ Sangs mêlés. - Outre les populations bantoues et négrilles, il y a au Congo quantité de peuplades et d'individus métissés, issus non seulement du croisement de ces deux éléments, mais encore des mélanges des sangs bantou, nigritien et éthiopien. Ces derniers métissages se sont naturellement faits dans le nord et le nord-est, au contact des peuples congoliens (Bantous), éthiopiens (Nubiens) et nilotes (Nigritiens du Soudan Oriental). Pour nous, les Sandés (Asandés ou Azandés) et les Mangbettous, présentent un intérêt spécial. - Chez les Azandés, on reconnaît des caractères des trois types avec prédominance, semble-t-il, des caractères généraux éthiopiens : taille moyenne, teint assez clair (brun chocolat), nez peu ou pas épaté, bouche petite et lippue, pommettes peu saillantes, joues rondes, tête peu 


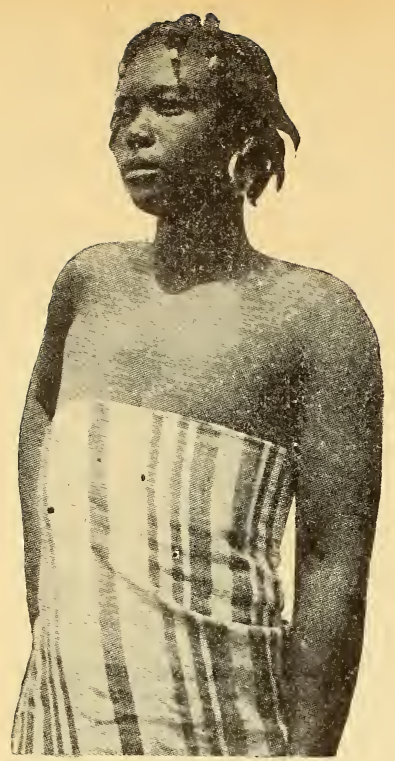

Fig. 82 - Femme Azaxdé.

allongée et assez large, démarche élégante et ferme, caractère indépendant. Les Azandés sont chasseurs et bons cultivateurs (bananes, manioc, mais, beau bétail); ils vivent en de gros villages. Ils pratiquent l'anthropophagie quoiqu'ayant du bétail. Ils sont artisans du fer, du cuivre, du coton, du cuir, portent des vêtements d'écorce, de cuir et de tissus. Leur organisation politıque et militaire, quoique toujours despotique, est plus élevée que celle des Bantous. Ils n'achètent plus leur femme et se la font désigner par le chef. Leur religion est un mélange de fétichisme et d'islamisme. - Les Mangbettous, mélange probable de Sandés, de Nilotes et de Bantous, constituent une peuplade puissante commandée par un despote sultan. Réputés hommes honnêtes, respectueux de la parole donnée, accordant à l'épouse les mêmes droits qu'à eux-mêmes et aimant le travail, agriculteurs patients, forgerons et potiers habiles, sculpteurs, excellents constructeurs de pirogues. En raison du respect professé pour la femme, la famille mangbettoue est d'organisation plus élevée et plus stable que celle des peuplades bantous.

Comme les Sandés, ils sont fétichistes islamisés et ont fortement subi l'influence de la civilisation arabe.

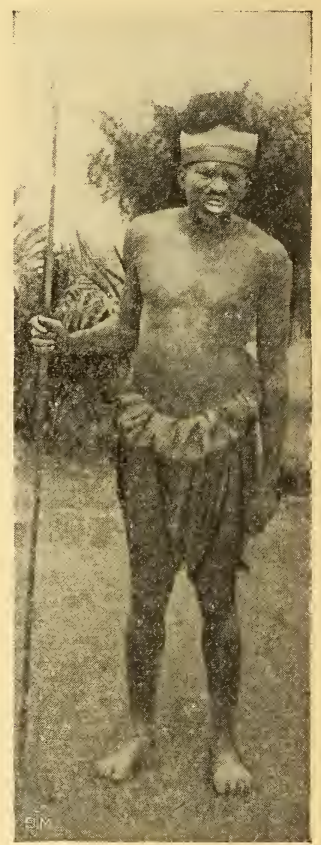

Fig. 83. - Chef Mangmettov. 
$\mathrm{X}$. - Meillevrs que nous ou pires. - Comment pouvous-nous résumer les principaux traits intellec'uels et mcraux de la population congolienne? Tout jugement porté sur le nègre doit être empreint d'inexactitude, sinon de partialité. En effet, les voyageurs nous rapportent le portrait de l'indigène africain, non tel qu'il est, mais tel qu'il ont cru le voir à travers le prisme de leur mentalité européenne. On peut affirmer que le nègre nous ressemble beaucoup, comme un homme à un autre homme, mais avec des différences qui trouvent ?eur origine dans la différence des milièux. Le milieu du nègre est la nature vierge, généreuse et rude des régions équatoriales. Le nôtre, loin d'être la nature encore vierge, porte au contraire l'empreinte profonde du développement séculaire d'une haute civilisation. Nous avons à considérer le nègre comme un homme plus rapproché que nous de l'état de nature, d'une mentalité autre que la nôtre, mais aussi valable et aussi respectable. Le noir, le jaune, le cuivré, sont des hommes exactement au même titre que le blanc.

Le nègre nous paraît enfant; il est sentimental et très spontané ; il est craintif, ce qui paralyse souvent en lui l'esprit d'initiative ; il est souvent impréroyant, vit dans le présent et non dans l'appréhension d'un avenir malheureux. Il se contente d'assez peu : la satisfaction de ses besoins immédiats. La terre féconde lui accorde assez facilement un entretien rudimentaire, aussi ne cherche-t-il guère à dérelopper ses ressources. Comme souvent l'homme civilisé, le nègre est bavard et aime les contes. Comme nous il est coquet, il aine se parer et, nous prenant pour être puissants et supérieurs à lui, il se pare volontiers de nos défroques. En cela comme en tant de choses, il cherche à nous ressembler. Certains explorateurs le disent fréquemment inhospitalier, vindicatif et essentiellement menteur, mais nous accorderons plutôt créance à d'autres qui l'affirment aimable lorsqu'on i'aborde simplement et honnêtement, qui le disent hôte très 
généreux et véridique de nature. Sa crédulité, disent-ils, traduit sa bonne foi, son mensonge trahit toujours un état de crainte, de méfiance et de défense, vis-à-vis de son interlocuteur blanc. Tous, nous savons que la crainte engendre le mensonge, défense du faible. L'on est d'accord pour reconnaître au nègre une très haute faculté d'adaptation. De sa psychologie sociale et individuelle semble ressortir trois tendances : celle du commerce, celle d'imitation et le désir illimité d'apprendre.

Ce désir s'extériorise par des séries de questions qui n'ont pas de fin lorsqu'on veut bien y répondre avec bonté, ce à quoi le nègre est très sensible. Ce désir d'apprendre est secondé à merveille par un esprit d'observation toujours réel et quelquefois très sagace. Ces tendances de l'esprit nègre que l'on retrouve quelquefois chez nous, Européens, nous montrent combien seuls les bons exemples et les bons procédés réussiront à élever notre frère congolien à un état de civilisation supérieur à celui où il se laisse vivre.

" Tout mon contact avec les noirs me les a montrés semblables à nous. Mis dans les mêmes conditions, dans toutes les mêmes conditions, le blanc et le noir réagiront de même. D'autre part, demeuré plus près de la nature, le noir congolais est d'intelligence pratique infiniment plus vive que la plupart des blancs. Il doit en effet se suffire très souvent à lui-même à peu près pour tout, et c'est toujours un spectacle poignant pour un penseur que de voir le noir aux prises avec les mille difficultés de la vie dans la brousse, aux mauvais passages d'eau par exemple, aux campements par mauvais temps, etc. Ils accomplissent alors de véritables tours de force d'ingéniosité et de labeur. Quand le blanc voudra bien reconnaître l'inanité de sa morgue vis-à-vis des autres couleurs, ces autres couleurs pourront lui devenir mieux connues, et par suite le travail commun en bénéficiera pour l'avantage commun. "(Lemaire, correspondance). 


\section{LE GOUVERNEMENT COLONIAL. - RÉGIME POLITIQUE, ADMINISTRATIF, JUDICIAIRE, FINANCIER}

I. - Le gouvernement. - La reprise du Congo par la Belgique se fit au cours des années 1907 et I908. Le 28 novembre 1907 le traité de cession fut conclu entre l'État Indépendant du Congo et la Belgique. Le 20 août Igo8, la Chambre des représentants et le 9 septembre 1908 , le Sénat votèrent l'annexion. Le 18 octobre suivant fut donné l'arrêté royal promulguant la lo1 d'annexion. A partir du I 5 novembre dernier (1908) la Belgique assura l'exercice de son droit souverain sur sa colonie. Jusqu'alors le Congo avait été pays de colonisation personnelle dénommé État Indépendant et gouverné par un souverain absolu, le roi Léopold. Celui-ci exerçait directement les pouvoirs législatif, exécutif et administratif. Il s'était entouré de divers services constitués en un gouvernement dit central, siégeant a Bruxelles et un gouvernement dit local, siégeant à Boma, ville capitale du Congo.

Le gouvernement central dirigé par un secrétaire d'État comprenait trois départements : affaires étrangères, finances et intérieur.

Le gouvernement local était dirigé par un gouverneur général représentant le roi en Afrique. Assisté d'un vice-gouverneur, d'un inspecteur d'État et d'un secrétaire général, il assurait l'exécution des décrets et décisions du souverain et avait la haute direction de tous les services. Le Congo est divisé en 14 districts à la tête desquels sont placés des commissaires 
de district. Ceux-ci sont secondés par les chefs de zone et de secteur et par les chefs de poste.

Actuellement, la colonie est administrée par un ministère des colonies ayant son siège dans la capitale de la métropole et remplaçant le gouvernement central. Le gouvernement local est devenu une administration locale. Des

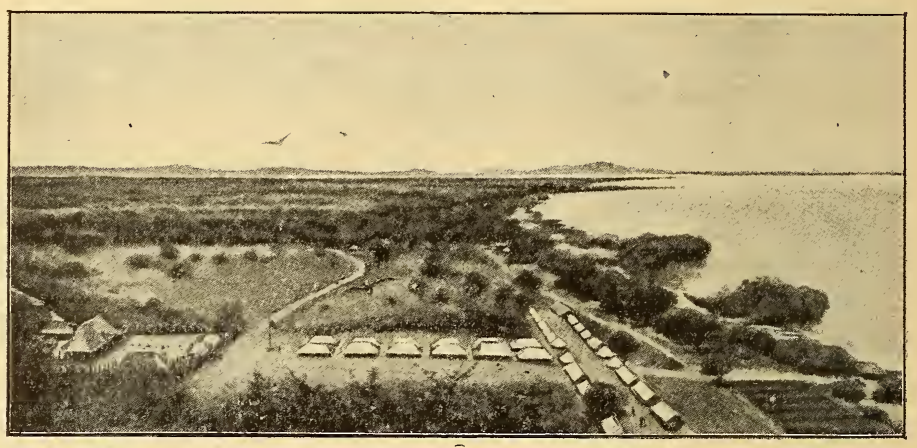

Fig. 84. - Panorama d'une station : MPwéto (Pwéto) en 1898 sur le lac M̃oéro. - A gauche, espace carré clôturé avec habitations assez vastes: quartier des Européens; au centre 12 cases disposées en deux rangées: quartier de la force publique ; - à droite, deux files de nombreuses cases moins grandes que celles des soldats: quartier des travailleurs; - entre les huttes des travailleurs et la berge du lac: jardins potagers. (Phot. Lemaire.)

Cette photographie, fort intéressante, constitue à elle seule une leçon de topographie urbaine qui pourrait s'appliquer à plus d'une de nos cités européenues : quartier aristocratique, militaire, populeux. Remarquer la route aboutissant sur la place entre le quartier européen et le quartier militaire.

changements fondamentaux seront probablement et à bref délai apportés dans le système des services locaux et dans les attributions des fonctionnaires en Afrique, en un mot dans toute l'administration locale en raison de la transformation du régime politique du Congo qui, naguère colonie personnelle autocratique, est devenue colonie nationale bénéficiant des garanties constitutionnelles de la métropole.

Les territoires soumis à la colonisalion beluge sont non seulement ceux de l'ancien État Indépendant du Congo, mais 


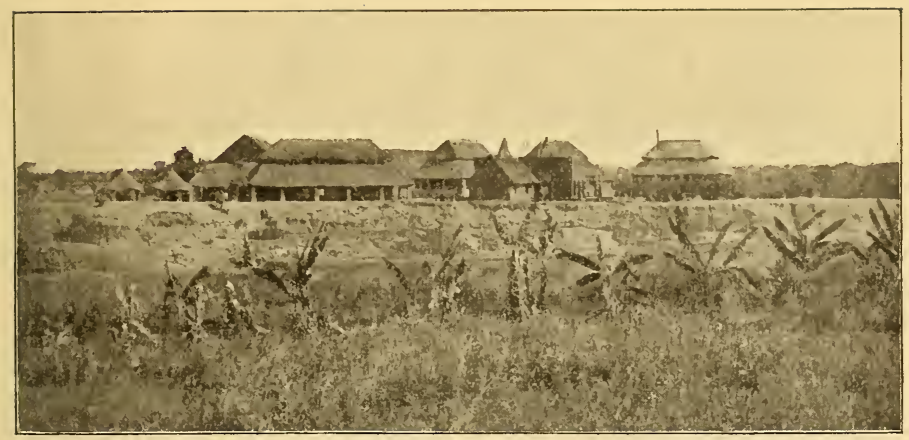

Fig. 85. - Vue générale d'une station militaire de l'État : DouxGou en I9o3. A l'avantplan : jeunes bananiers. (I'hotogr. Lemaire.)

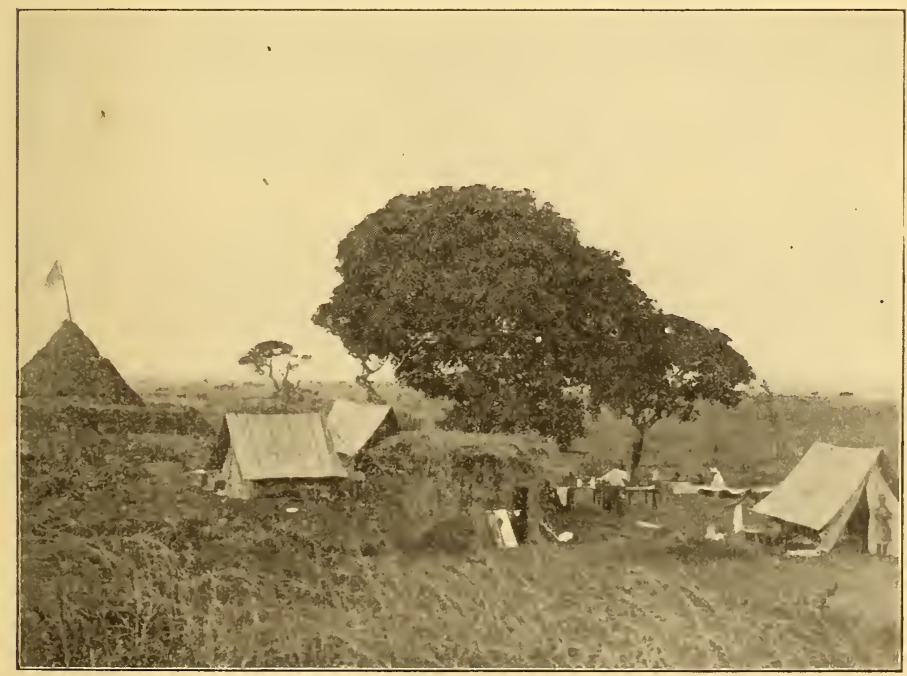

Fig. 86. - Sur la ligne de faite Congo-Zambèze pres du lac Dilolo. Canipenent DE LA mission Scientifique du KAtanga. Au second plan, à gauche, butte géodésique établie par la mission. A l'arriere-plan à droite, on aperçoit le lac. Remarquer le caractere de haute plaine et l'aspect du tapis végétal. (Photogr. Lemaire.) 
encore ceux pris à bail à l'Angleterre par le dit État par la convention de I894: l'enclave de Lado qui fera retour à l'Angleterre à la mort de Léopold II et la petite enclave de Mahagi sur le lac Albert dont le bail ne sera pas rompu par suite de la mort du roi. Les conventions de I894 et de 1907

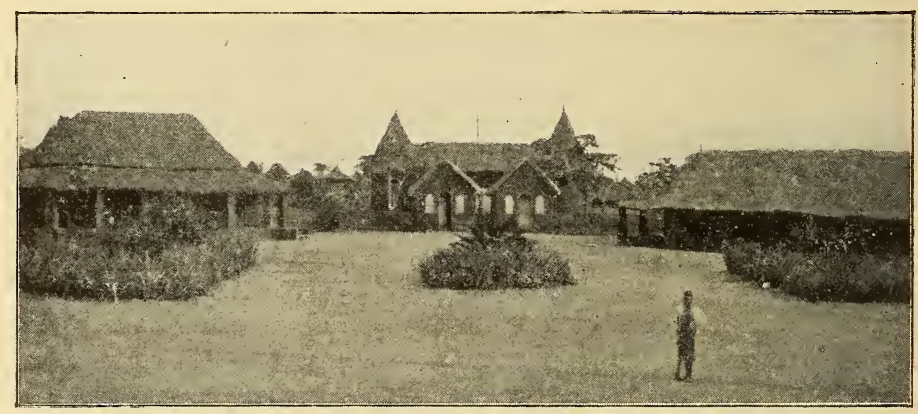

Fig. 87. - Intérieur d'un poste militaire de l'État : FARADJ, station fortifiée. Au milieu, à l'arrière-plan, porte d'entrée avec corps de garde, prison, etc.; à gauche au second plan: maison du chef de zone; à droite au second plan: bâtiment pour blancs, avec magasin; à l'avant-plan : factionnaire soldat nègre de la force publique. (Photogr. Lemaire.)

reconnaissent à la Belgique le droit d'en bénéficier aussi longtemps qu'elle possédera le Congo. L'enclave de Mahagi présente un intérêt considérable car elle ouvre à la colonie belge un débouché par la voie du Nil. Il est vrai que la navigation y est interrompue par le sedd, mais ce n'est là qu'un obstacle temporaire qui sera vaincu dans un délai rapproché par la colonisation anglaise du Soudan nilien.

II. LA POPULATION BLANCHE. - La colonisation active sur le sol africain se fait par un nombre croissant d'Européens de diverses nationalités, aidés par une multitude de travailleurs noirs.

$\mathrm{Au} \mathrm{I}^{\mathrm{er}}$ janvier 1908, il y avait sur la terre congolienne 2,943 blancs parmi lesquels I,743 Belges, 200 Suédois, 197 Italiens, I45 Anglais, I29 Portugais, I24 Hollandais, 88 
Suisses, 58 Françaıs, 57 Allemands, 55 Russes, 54 Norrégiens, 47 Américains, 36 Danois, 25 Luxembourgeois, I4 de nationalités diverses.

I] n'est guère possible de se faire une idée même approximative de la répartition des blancs en considérant leur classement par districts, ceux-ci étant des divisions territoriales administratives de dimensions extrêmement variables. Les groupements de plus de 25 blancs sont peu nombreux et

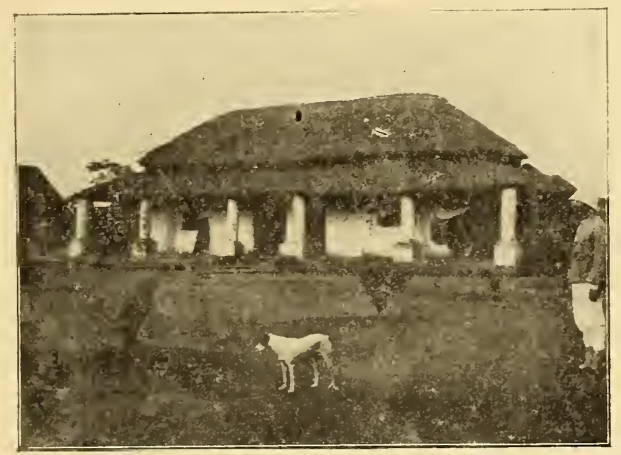

Fig. 88. - TYPe dune habitation potr Européen. Remarquer le double toit de chaume et la galerie abritée par le toit débordant. (Photogr. commun. par M. Lemaire.)

varient d'ailleurs beaucoup d'une année à l'autre suivant les exigences des services d'administration et d'exploitation. En 1908 - $\mathrm{I}^{\text {er }}$ janvier - Boma comptait 262 blancs, Léopoldville 208, Matadi 155, Stanleyville 83, Kindou 72, Dima 49, Thysville 38, Nourelle-Anvers 36, Banana 35, Kinchasa 32, Pwéto 30, Coquilhatville 29, Toumba 27, Kisantou (mission située non loin du chemin de fer, à moins de roo kilomètres de Léopoldville) 26.

Parmi cette population, la mortalité fut grande dans les débuts de la colonisation. La connaissance du milieu aui se traduit par toutes espèces de pratiques individuelles et de mesures collectires, fait diminuer cette mortalité d'année en 
année. En 1902 elle atteignait 6.31 p. c., en 19035.09 p. c., en 19044.23 p. c., en 19053.4 I p. c., en 19062.85 p. c., en 19072.97 p. c. Le choix d'un personnel d'élite au double point de vue physique et moral s'impose comme facteur essentiel de la diminution de la mortalité des colons.

III. Les CUltes. - La liberté de conscience et la tolérance religieuse sont garanties par l'Acte général de Berlin. L'organisation religieuse est d'initiative privée avec l'approbation du gouvernement.

340 missionnaires catholiques belges des deux sexes et 2 I I missionnaires protestants des deux sexes administrent des missions religieuses drınt le but est d'évangéliser, d'instruire, de civiliser les noirs. Leur principal moyen d'action est l'établissement de colonies scolaires, de lazarets, de dispensaires, de refuges, de fermes-chapelles, ctc. D'après les voyageurs les plus dignes de foi, leur influence est bienfaisante.

IV. LA FORCE PUBliQUe. - La force publique ( 13,750 hommes) recrutée par levées et par engagements a pour but d'assurer la police intérieure de l'État.

V. LA Justice. - Le pouvoir judiciaire se résume en ceci : en matière civile et commerciaie, les différends gont tranchés par les tribunaux de l'État excepté dans les cas où les deux parties en cause sont indigènes, auquel cas le chef local juge selon la coutume; en matière répressive, toute infraction à la loi pénale commise par un blanc ou un indigène est jugée par les tribunaux de l'État. Dans certains cas, les indigènes peuvent être soumis à l'application des coutumes indigènes.

VI. - Les FinAnces. - La Dette. - La valeur totale de la dette congolaise s'élevait à I I4,576,650 francs au moment de la reprise de la colonie par la Belgique. 
Cette dette comporte une charge annuelle de 4,510,705 francs. Elle a été contractée par une série d'emprunts de 1887 à I906. Nous renvoyons le lecteur à la partie historique de ce court aperçu pour lui rappeler la genèse financière des rapports entre la Belgique et l'État du Congo.

Le budget arrêté de 1908 se marquait par un léger excédent des recettes ordinaires, 35,378,000 francs, sur les dépenses ordinaires, 35,344,088 francs. Les dépenses extraordinaires prévues s'élevant à 3,90I,875 francs, il restait 3,866,, 63 francs à couvrir par la voie d'emprunt.

Le budget arrêté de 1909 se différencie en un budget métropolitain destıné à couvrir uniquement les dépenses que l'administration de la colonie exige dans la métropole (ministère des colonies) - ces dépenses s'élèvent à 942,450 francs - et un budget colonial local. La recette ordinaire balance la dépense ordinaire: 36,094,036 francs. Une somme de $\delta, 423,300$ francs consacrée à des dépenses extraordinaires sera demandée à l'emprunt.

Les impôts.-- Les ressources ordinaires de la colonie, ou mieux de l'État du Congo, jusqu'à la reprise, ont été $\mathrm{I}^{\circ}$ des contributions directes et indirectes sur les non-indigènes; $2^{\circ}$ les douanes; $3^{\circ}$ - part la plus considérable - contributions sur les indigènes, payables en argent, en nature ou en prestations. Elles étaient payables en général par douzièmes, l'argent d'impôt ne pouvant sortir des limites 6 à 24 francs par tête, le travail à fournir en manière d'acquittement d'impôt ne pouvant excéder 40 heures par mois. En I 908, 975,000 indigènes étaient inscrits aux rôles des prestations. La grande majorité travaillatt à la récolte des produits domaniaux (caoutchouc et copal), au pcrtage, etc. Un grand nombre encore payait les impôts en nature. Enfin, une très faible minorité, 85,000 , remettait en argent le montant de ses contributinns. 
La réforme du système d'imposition indigène s'est montrée comme une des plus urgentes. La question d'introduction ef de généralisation de l'emploi du numéraire est intimement liée à celle des impôts. Nous en parlerons un peu plus loin.

La monnaie. - Le système officiel des monnaies, poids et mesures de l'État du Congo était le même qu'en Belgique. Par une convention toute récente, la colonie belge est incorporée à l'Union latine : les monnaies belges d'or et d'argent ont cours dans la colonie. Par mesure de transition, les monnaies frappées par l'État Indépendant du Congo auront encore cours un certain temps. Le billon qui sera frappé spécialement pour la colonie n'aura pas cours en Belgique.

L'emploi du numéraire s'introduit avec une lenteur extrême parmi les indigènes. De nombreux essais locaux ont pourtant été couronnés de succès. Il en est de même au Gabon (Congo français), en Afrique occidentale française, où l'emploi du numéraire, encore tout à ses débuts, facilite étonnamment les transactions commerciales. Toutes les tentatives et spécialement le fait qu'aux environs des stations - tant au Congo belge que dans les colonies étrangères - l'indigène se familiarise petit à petit avec l'emploi du numéraire, montrent toute la nécessité qu'il y a de payer en argent les produits ou le travail de l'indigène.

VII. LE RÉGIME FONCIER. - Pour une exploitation rapide, une appropriation économique du sol s'imposa à l'administration de l'État Indépendant du Congo. Avant la reprise, en 1908 encore, cette appropriation, par une série de nombreux décrets souverains, se trouvait réglée ainsi qu'il suit :

I $^{\circ}$ Domaine public, hors du commerce, pas susceptible de propriété privée, plus particulièrement affecté à des services publics, consistant en rivières, cours d'eau navigables ou flot- 
tables avec leurs rives, chemins, chemins de fer, routes, rivages, lais et relais de la mer, ponts, forteresses, etc.

$2^{\circ}$ Domaine privé (D.P.) originairement constitué par toutes les terres que possédaic l'Etat et qui étaient susceptibles d'appropriation privée, c'est-à-dire que l'État avait le droit de céder en pleine propriété, en location, en usufruit ou en concession. Ce domaine privé comportait toutes les terres vacantes - res mullius (I) - c'est-à-dire non occupées par les indigènes ou non possédées par de non-indigènes. Une partie très considérable de ce domaine privé fut aliénée au profit de la Fondation de la Couronne et de sociétés d'exploi. tation concessionnaires. Une autre partie considérable fut exploitée en régie par l'État du Congo. Le restant attendait la mise en valeur soit en régie, soit en concession.

Un décret souverain du 3 juin 1906 détacha du domaine privé toutes les terres exploitées en régie et toutes les mines non encore concédées pour en constituer le domaine national. Il en résıltait qu'à partir de la promulgation de ce décret, le domaine privé de l'Etat du Congo consistait en toutes terres possédées par l'État, à l'exclusion de celles administrées en régie et des mines déià concédées.

$3^{\circ}$ Domaine national, formé en exécution du décret précité par toutes les terres administrées en régie et toutes les mines non concédées à la date du 3 juin I go6. D'importantes parties de ce domaine ont été aliénées en pleine propriété ou en usufruit ou en concession au profit de particuliers, de sociétés, de missions ou encore de la Couronne.

$4^{\circ}$ Domaine de la Couronne, constitué, étendu, organisé, réglementé en exécution d'une série de décrets souverains promulgués de 1896 à 1904. Formé d'une partie déterminée du clomaine privé, il était la propriété de la Fondation de la Couronne qui jouissait de la personnalité civile. Différents décrets apportèrent des modifications au domaine de la Cou-

(1) Littéralement : chose de personne, n’appartenant à personne. 
ronne et concédèrent à des sociétés des droits d'exploitation sur une grande partie de ses territoires. L'État du Congo se chargea d'exploiter les terres de la Couronne de même que celles de certaines concessions (Abir et Mongala) (décret du 22 décembre 1906).

Enfin, au cours des pourparlers entre l'Êtat Indépendant du Congo et la Belgique au sujet de la reprise de la colonie, un décret souverain fut promulgué le 5 mars 1908 retirant à la Fondation de la Couronne la personnalité civile à partir du jour où la Belgique assumerait l'exercice de la souveraineté du Congo, ses biens devant faire retour au souverain Léopold. Celui-ci, moyennant des engagements de la Belgique lui céderait à la dite date les terres vacantes du domaine de la Couronne - les concessions faites étant respectées - ainsi que le porteteuille et des immeubles de la Fondation. Le Roi-Souverain se réservait 40,000 hectares dans le Mayoumbé.

Ainsi donc, en vertu de ce décret, le domaine de la Couronne fut supprimé de droit et de fait par la reprise de la colonie par la Belgique - ( I 5 novembre I908, date à laquelle la Belgique assuma l'exercice de la souveraineté du Congo).

$5^{\circ}$ Terres acquises par les non-indigènes, particuliers, sociétés ou missions. Ces terres ont été achetées aux indigènes ou à l'État ou bien elles ont été données en bénéfice de location ou de concession par l'État qui les détacha ainsi de son domaine privé. Quelques-unes enfin furent attribuées en don à des missions religieuses.

$6^{\circ}$ Terres appartenant aux indigènes, propriétés des indigènes en vertu de leur droit d'occupation. En général et à cause souvent de l'état semi-sédentaire, semi-nomade du cultivateur nègre, ces terres n'ont pas été délimitées. Des décrets souverains ont bien été promulgués ordonnant cette délimitation, mais les difficultés d'exécution de ces décrets sont extrêmes. 
L'annexion du Congo étant chose accomplie, le régime foncier de la colonie se trouve modifié. Existent encore: $:^{\circ}$ le Domaine public, $2^{\circ}$ le Domuine privé avec le Domaine

ESQUISSE DE L'APPROPRIATION FONCIÈRE

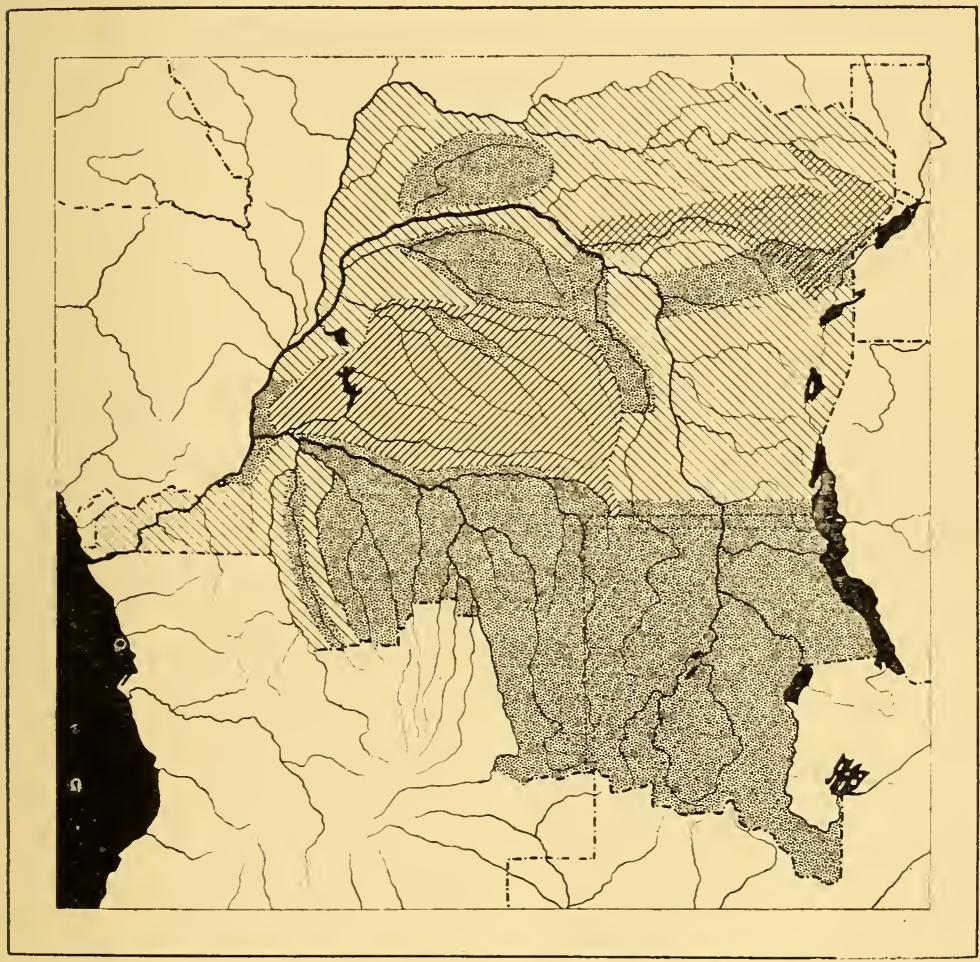

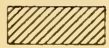

Ancien domaine de la Courome

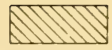

Domaine prize avec domaine national

Fig 89

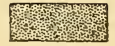

Territrires

concédés

Échelle 1 à 25.000 ono

national, $3^{\circ}$ les terres acquises par de non-indigènes, $4^{\circ}$ les terres appartenant aux indigènes. Le principe fondamental de cette appropriation des terres gît dans le mode d'exploiation que l'on a cru nécessaire d'appliquer aux terres congo- 
liennes. Ou bien l'État exploitait d'une part en régie le domaine national, pour son propre compte, d'autre part le domaine de la Couronne pour le compte de la Fondation de la Couronne et certaines terres concédées pour le compte des sociétés concessionnaires elles-mêmes (Anglo-Belgian-India Rubber ou Abir dans le district de l'Équateur, bassin du Lopori et de la Maringa, et Mongala dans le district des Bangalas, bassin de la Mongala) ou bien les sociétés concessionnaires et les propriétaires privés exploitaient directement les territoires dont ils avaient la concession ou la propriété. Il pouvait aussi se produire que cette exploitation se fît en association mutuelle, voire avec l'État.

La nationalisation belge de la colonie qui fut l'État Indépendant du Congo entraînera forcément des réformes considérables dans son organisation administrative, judiciaire et financière. Parmi ces réformes, les modifications à apporter au régime foncier sont les plus urgentes parce qu'elles concernent le plus directement la situation de l'indigène et ses rapports vitaux avec le blanc. 


\section{LA SITUATION ÉCONOMIQUE DE LA COLONIE}

I. - Exploitation FORestière et Agricole. - La forêt, la savane et la brousse, chacune arec sa nature de sol et son climat, ont leurs produits spécifiques. Parmi ces produits, il en est auxquels le marché du monde assure un écoulement constant, quelle qu'en soit la quantité. Nous voulons parler des bois de construction et d'ébénisterie, des gommes comme le caoutchouc, des résines comme le copal, de certaines huiles, du cacao, du café, du coton et aussi des céréales que le Congo peut produire en abondance. Les gros problèmes qui apparaissent d'emblée sont ceux de la main d'œuvre et des voies de transport.

Les bois. - La grande forêt — qui n'eśt d'ailleurs pas partout de même renue et présente çà et là des éclaircies, des clairières plus ou moins étendues — se trouve la plus puissante dans le Mayoumbé, la Loukoléla, l’Arouwimi et la Province Orientale. Elle constitue une source inépuisable de bois de construction et d'ébénisterie.

Les bois propres à la construction, souvent jaunâtres, sont de forte densité (jusqu'à 950) et d'une très grande résistance. Certain bois de densité à peu près égale à celle du chêne (725) dépassent très notablement celui-ci comme résistance. Nous ne citerons que le séké, l'élongo, l'éloukou, le moukoutou qui sont parmi les meilleurs. Les bois d'ébénisterie, noirs, 
rouges, jaunes et blancs, sortes d'ébènes, d'acajous, de palissandres (bois des îles) fournissent les meubles les plus beaux et les plus durables. Le plus remarquable est le mbotou, espèce de bois de fer.

Les essences colorantes sont nombreuses. Elles fournissent le rouge, l'amarante, le violet, le bleu, le brun. Signalons aussi l'orseille, plante parasite des arbres, fournissant un rouge-violet et le rocou, petit arbre donnant une teinture rouge. Les produits tinctoriaux végétaux sont de moins en moins demandés sur le marché et sont donc de peu d'importance. pour le Congo.

La région du Katanga fournit en quantité le bambuu dont l'utilisation peut être très grande. Sa croissance extrênement rapide lui donne une importance qui ne doit pas échapper dans la recherche de matières premières servant à fabriquer le papier.

Certains palmiers sont utilisés dans toutes leurs parties : fibres, feuilles, fruits (l'elä̈s de Guinée, le raphia vinifera dont les tibres servent à faire des étoffes indigènes, les pétioles des feuilles et les feuilles à construire et à couvrir les cases indigènes; les calamus qui donnent les rotangs, les attalea qui donnent le piassava à faire les brosses). D'autres essences spontanées sont utilisées de façons très variées : les gommiers, les cotonniers, le baobab, etc. Nous en parlerons sous les rubriques gommes, résines, textiles, plantes médicinales, etc.

Les bois de construction et d'ébénisterie ont été exploités en d'assez bons débuts, mais actuellement cette exploitation est interrompue et ne pourra être reprise effectivement et avec succès que lorsque le transport des matières pondéreuses et encombrantes destinées à l'exportation sera d'un prix moins élevé. I_e déboisement conduit rationnellement n'épuisera pas la richesse forestière et conservera au sol toute l’humidité nécessaire. 
Les huiles. - Le principal producteur d'huile est l'élä̈s de Guinée, palmier de belle venue - 25 à 30 mètres. Ses régimes ou grappes de fruits dépassent quelquefois I mètre de long et 40 kilos en poids. Sa croissance spontanée et exubérante sur presque tout le territoire de la colonie ne réclame

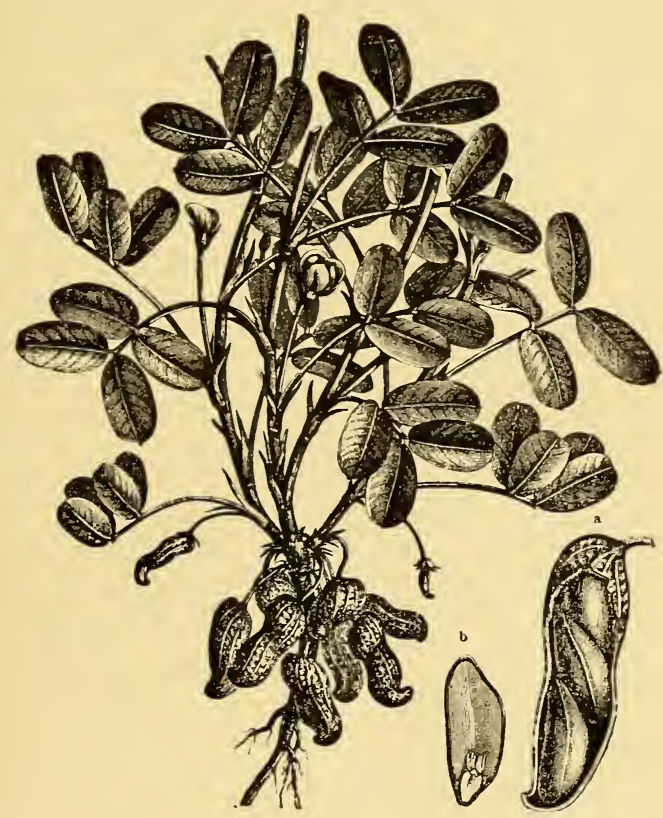

Fig. 90. - ARACHIDE; a) gousse avec fruit mûr, b) coupe du fruit mûr. (D'après le Bilder-Atlas zur Pflanzengeographie de Kronfeld, - Bibliogr. Instit. Leipzig.)

pour ainsi dire aucun soin. Les indigènes en font d'énormes plantations. Ses fibres constituent une excellente matière textile; ses feuilles sont utilisées comme couverture des huttes; sa sève fournit le malafou, boisson fermentée; son gros bourgeon terminal ou cœur dit choux palmiste est consommé comme légume; son fruit — plusieurs centaines d'amandes agglomé- 
rées en régime - donne des huiles industrielles et comestibles; de la partie extérieure, charnue, on extrait une huile épaisse employée dans la fabrication des bougies, des savons et dans le graissage des machines; du noyau de l'amande on extrait une excellente huile fine.

Le palmier à vin (Raphia vinifera) et le cocotier (Cocos nucifera), l'arbre à beurre (Karité), le ricin, le sésamier et d'autres plantes abondantes ne sont actuellement presque pas exploités comme producteurs oléagineux. I_as indigènes pratiquent beaucoup et partout la culture de l'arachide, petite plante donnant une graine assez volumineuse et très huileuse. Le rendement en est bon, elle donne environ 30 p. c. de son poids d'huile fine, d'usage combustible ou industriel et fournit un résidu apprécié comme engrais du sol et comme nourriture du bétail. La culture de. l'arachide ne réclame que des soins très rudimentaires; elle est très productive.

Les gommes et résines. - La plus grande richesse du Congo est celle du caoutchouc. Le caoutchouc est un produit de coagulation d'un suc qui n'est point la sève, qui circule dans la partie profonde de l'écorce de certains végétaux : arbres, arbrisseaux et lianes et que l'on appelle latex. C'est une liane forte et longue appelée Landolphia - il y a plusieurs variétés de landolphia - qui fournit le caoutchouc en pıus grande quantité et en meilleure qualité. Un arbre l'iréh, de 20 mètres de haut fournit aussi un caoutchouc abondant et apprécié. Le caoutchouc est exploité non seulement en forêts, mais en plantations. Celles-ci constituent une des principales activités coloniales du Congo et peuvent se faire sur presque toute l'étendue de la colonie. La région du Kasaï est jusqu'à présent la plus riche et fournit le caoutchouc rouge du Kasaï désigné dans le commerce sous le nom de Kasä̈ prima; c'est le meilleur (fr. I2.50 à fr. I3.50 le kilo). Le gouvernement de l'État du Congo a pris de bonne heure les mesures néces- 
saires non seulement pour éviter l'épuisement des ressources en plantes à latex, mais pour les augmenter et les améliorer.

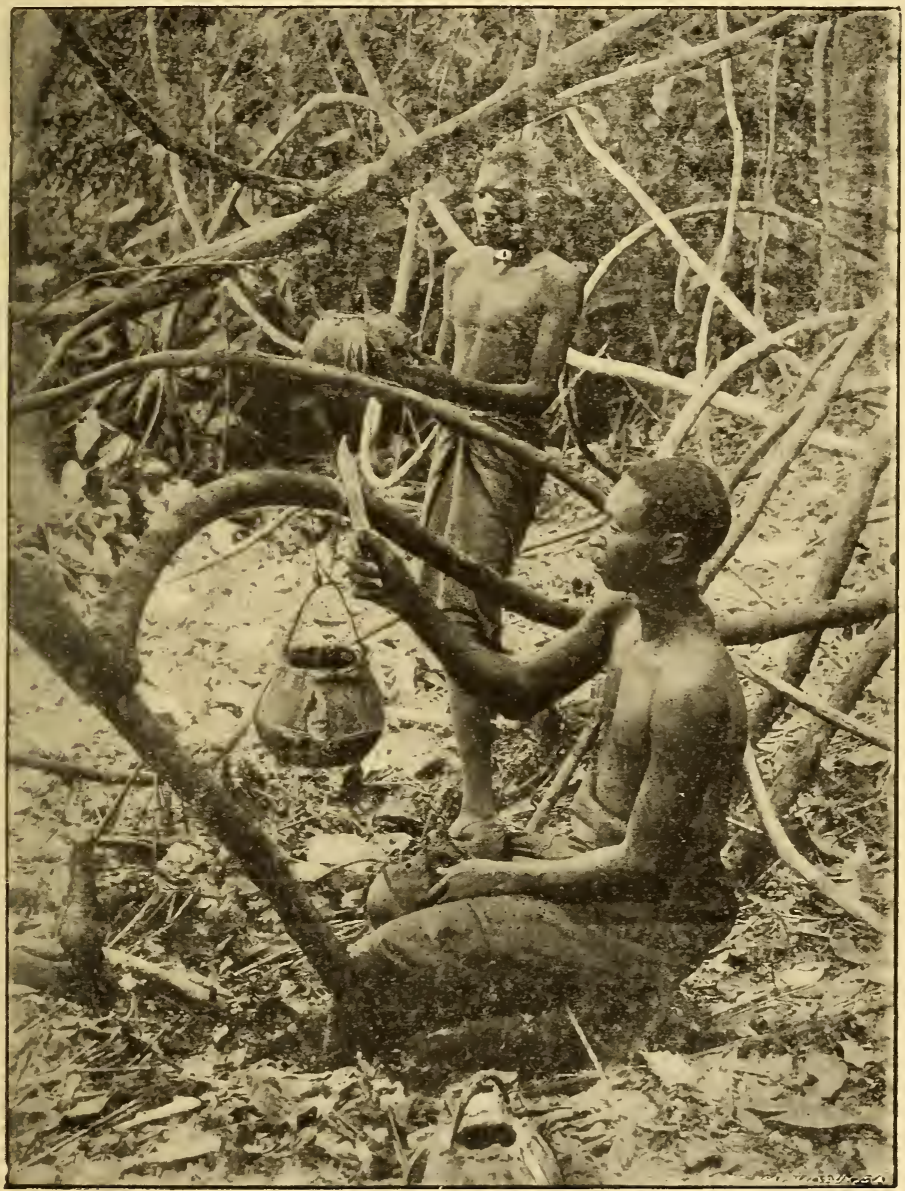

Fig. 9I. - LA RÉCOLTE dU CAOLTCHOUC.

D'une part l'exploitant est obligé de replanter en proportion de sa récolte et d'autre part des essences étrangères ont été introduites, telle l'Herea du Brésil qui donne le caoutchouc 
Para, le plus apprécié de tous. Les trois plus importants centres de culture d'essences caoutchoutifères, qu'il importe

\section{L'EXPORTATION DU CAOUTCHOUC DE I887 A I907}

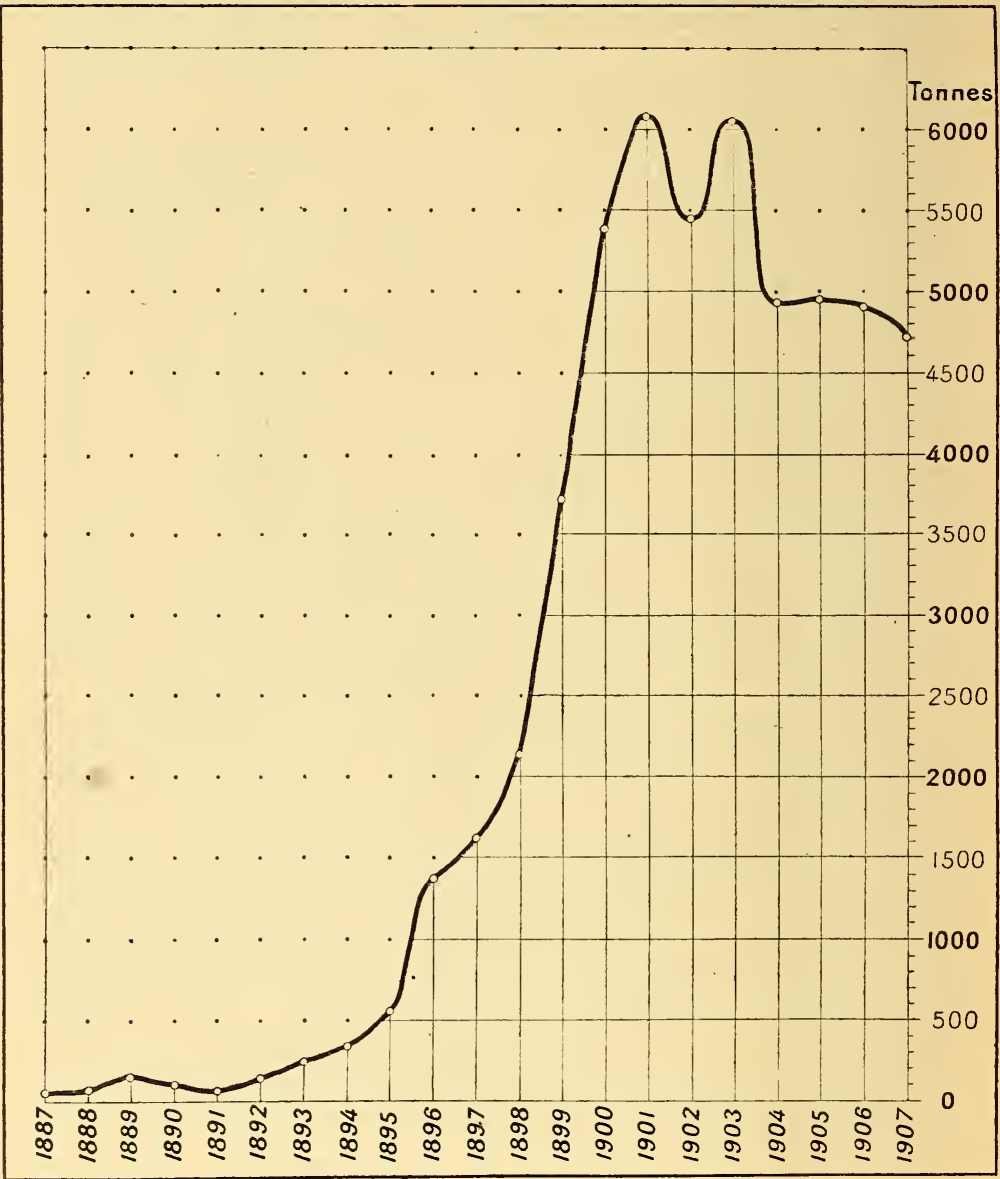

Fig. 92.

(D'après les statistiques officielles)

de ne pas confondre avec les très nombreux centres de production, étaient jusqu'en ces derniers temps celui de Banza 
dans le Mayoumbé, celui de Douma dans l'Oubanghi et celui de Lodja sur la haute Loukénié dans le Loualaba-Kasaï. L'activité de l'État dans l'entretien et le développement des richesses de la colonie se manifestera bientôt dans l'organisation des plantations à caoutchouc. Les centres de culture d'essences caoutchoutifères seront considérablement multipliées et pourront soutenir une production constante.

En I90I, la production du caoutchouc congolien a atteint plus de 6,000,000 de kilogrammes. Depuis elle a diminué.

Sur les 68,000 tonnes négociées sur le marché mondial en 1906, 43,000 tonnes étaient originaires d'Amérique, 23,000 d'Afrique $(4,849$ tonnes du Congo belge d'une valeur de 48,489,000 francs, soit les $5 / 6$ du commerce d'exportations congoliennes) et 2,000 d'Asie, de l'Insulinde et de la Polynésie. Les principaux marchés d'importation sont ceux de New-York, de Liverpool, de Hambourg, d'Anvers et du Havre

Copal. - Gomme employée dans la fabrication des vernis, le copal est une des richesses du Congo. Fourni par des arbres très abondants, 11 a atteint en 1906 une exportation de 869 tonnes, soit une valeur de I,086,000 francs.

Gutta-percha. - Des essais de culture d'arbres à guttapercha ont réussi.

Les textiles. - Comme cultures industrielles, il faut encore signaler les cotonniers qui d'ailleurs croissent spontanément et dont la culture limitée aux régions à saison sèche promet un avenir rémunérateur. Rappelons les palmiers et le baobab dont les fibres sont utilisées comme textiles par les indigènes ou par l'industrie moderne (brosserie, vannerie), le chanvre, narcotique aimé des indigènes du Kasaï, dont le rendement permettra l'exploitation comme textile. La ramie et le jute récemment introduits au Congo y ont trouvé de très bonnes conditions de déreloppement. 
Les cultures alimentaires sont celles du manioc, de la patate douce, de l'igname, du sorgho, du millet, de l'éleusine, du riz, du mais, de la banane, de l'arachide, des courges, de la canne à sucre, des légumineuses qui toutes entrent

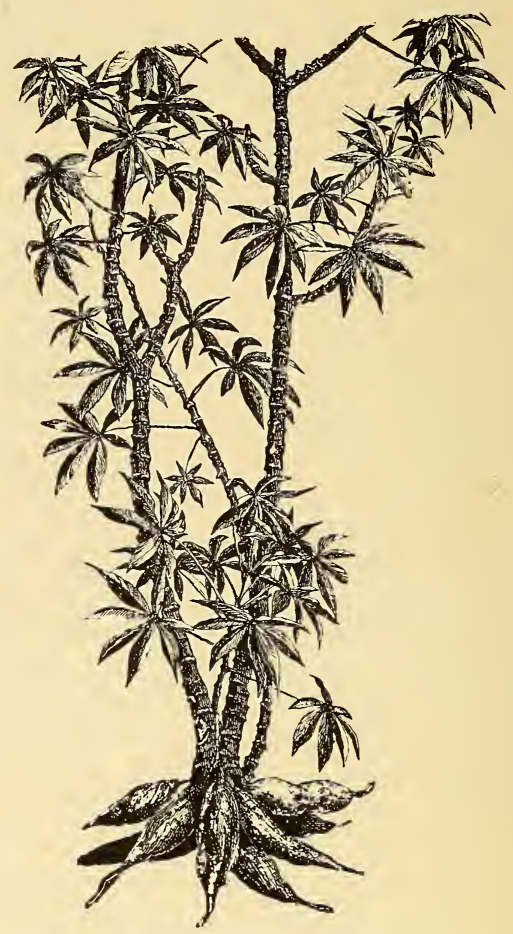

Fig. 93. - Manioc. - Racine comestible, utilisée non seulement par l'indigène, mais encore par l'Européen qui en consomme sous forme de tapioca et de fécule.

(D’après le Bilder-Atlas cité de Kronfeld.)

pour une part considérable dans l'alimentation locale. On a introduit le froment dans le sud-est et l'est. - Manioc: dans toute la colonie; rendement jusqu'à 40,000 kilos à l'hectare soit l'alimentation annuelle de 40 indigènes; sert à la fabrication du tapioca et de la fécule. - Patate douce et igname: 
tubercules comparables aux pommes de terre; dans presque toute la colonie; grand rendement. - Sorgho,céréale donnant de bonne farine et de bonne bière (pombé); surtout dans le nord, l'est et le sud-est (savane). - Millet et éleusine : céréales, nourriture de l'indigène et du bétail : dans l'est et le nord. - Riz: céréale répandue et cultivée avec succès dans la plus grande partie de la colonie (riz des marais et surtout riz des montagnes demandant moins d'eau). Maïs : céréale dans tout le Congo; fort rendement; nourriture très appréciée de l'indigène; excellent fourrage. Banane: le bananier (musacée) plante herbacée de grande taille : 5 mètres; fruits en régimes de 20, 30 kilos et plus; nourriture délicate et supérieure; rendement: I0,000 pieds sur un hectare produisent 75, 00 kilos (voir fig.47).-Arachide: voir plus haut sous la rubrique "huiles». - Elä̈s guineensis: voir plus haut. - Les légumineuses d'Afrique et d'Europe et toutes plantes potagères fournissent une alimentation abondante aux Européens et aux indigènes dans le voisinage des stations (voir plus haut : cultures indigènes).

Outre ces cultures dont les produits jusqu'à présent ne servent qu'à l'alimentation locale des noirs et des blancs, il est trois cultures coloniales d'alimentation de rendement appréciable : la canne à sucre, le cacaoyer et le caféier. Canne à sucre : grande graminée, croît spontanément et est cultivée par l'indigène dans tout le haut Congo. Quoique d'un bon rendement, elle ne pourra pas lutter contre la grande production des îles Hawaï et de l'Amérique. Cacaoyer ou théobrome (=aliment des dieux): arbre de 5 a Io mètres de hauteur, originaire d'Amérique; beaucoup de variétés de culture; introduit en I884 dans l'État du Congo; de culture très difficile et attaqué par beaucoup d'insectes il est surtout propagé dans le Mayoumbé, moins dans l'Équateur, l'Arouwimi et la Province Orientale. Actuellement il y a. environ au Congo 300,000 cacaoyers (exportation en 1896 : 


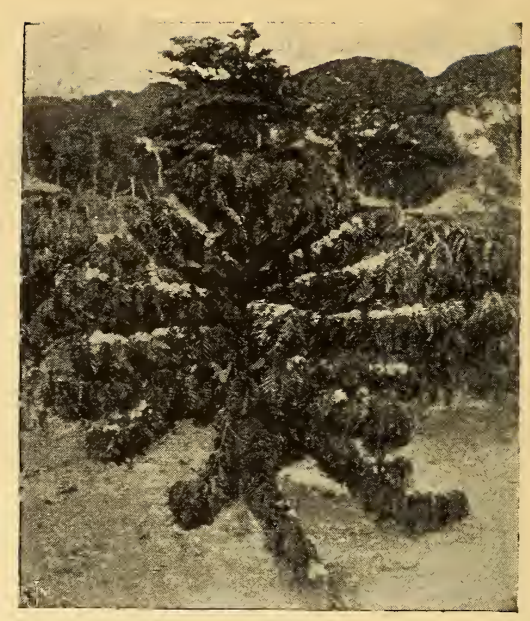

Fig. 94.-Ux CAféter d'Afrique (coffea liberica). L'albuste est en fleur.

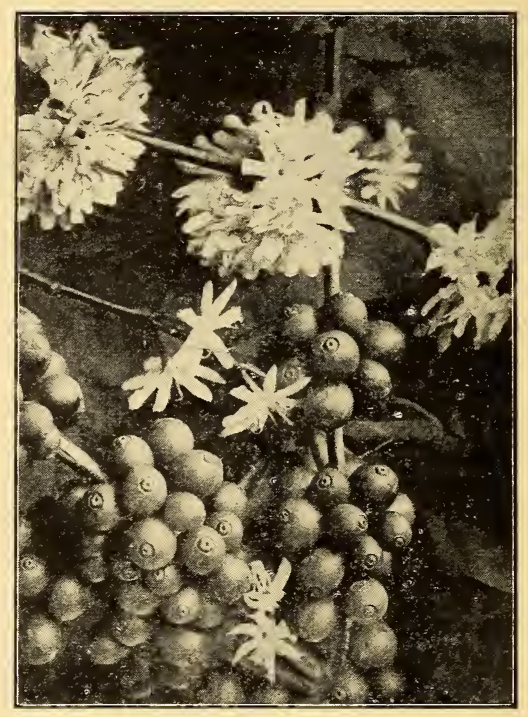

Fig. 95 - Fleuk et Fruit de Caférer (d'après Vegetationsbilder de Kürsten ct Schenc'k; G. Fischer à léna). 
92 kilos; en 1906: 402,429 kilos). - Caféier: petit arbre originaire d'Afrique; beaucoup de variétés; de culture plus aisée; sa culture a commencé en I894; il est principalement répandu dans le Mayoumbé, dans la zone des Stanley-Falls, dans l'Équateur et l'Arouwimi. En I904, l'exportation a atteint le chiffre encore très modeste de I6I,522 kilos d'une valeur de 153,446 francs; depuis, la production a basssé : en 1906, 74,916 kilos, d'une valeur approximative de 63,000 fr. La situation de la culture du café est difficile en raison de l'énorme production du Brésil : I2,300,000 balles de 60 kilos en 1906, soit 738,000,000 kilos, les trois quarts de la production du monde $988,800,000$ kilos.

A classer aussi parmi les cultures d'alimentation, celles des plantes à fraits suivantes : ananas, papayer, arbre à pain, jacquier, safoutier, avocatier, cœur de bœuf, goyavier, maracoujas, pomme cannelle, grenadier, pomme d'acajou, tomate, aubergine, citronnier, mandarinier, oranger, manguier et celles des plantes à épices : poivrier, gingembre, muscadier, piment, cannellier, vanillier, etc... dont beaucoup croissent spontanément dans la forêt.

Les plantes médicinales et aromatiques sont nombreuses :

Les plantes médicinales et aromatiques sont nombreuses: kolatier, euphorbes, tamarinier, quinquina, camphrier, croton, baobab, liane à réglisse, ricin, etc.

Enfin la culture du tabac déjà étendue par l'indigène à de très grandes régions, pourra, dans l'avenir, prendre une extension considérable en vue de l'exportation.

Nous n'avons énuméré que les produits végétaux principaux spontanés ou cultivés. En vérité il y a un très grand nombre d'exploitations et de cultures qui actuellement commencent. Forcément le choix le plus avantageux se fera, certaines cultures seront abandonnées, mais d'autres, nouvelles, 
seront introduites et soumises à des essais. La station agricole d'expériences d'Éala répond en partie à cette destination. Elle fut créée en 1900 et comporte actuellement un jardin botanique, un jardin d'essais et une ferme modèle. Sans exagération et sans faux espoir, on peut croire que le rendement des cultures du Congo d'ici à 50 ans, dépassera ce que les plus audacieux ont prédit, à plusieurs conditions bien entendu qui ressortiront au cours de notre étude.

Les animaux. - Jusqu'à présent, les produits de la chasse servent à l'alimentation locale de la population. Les

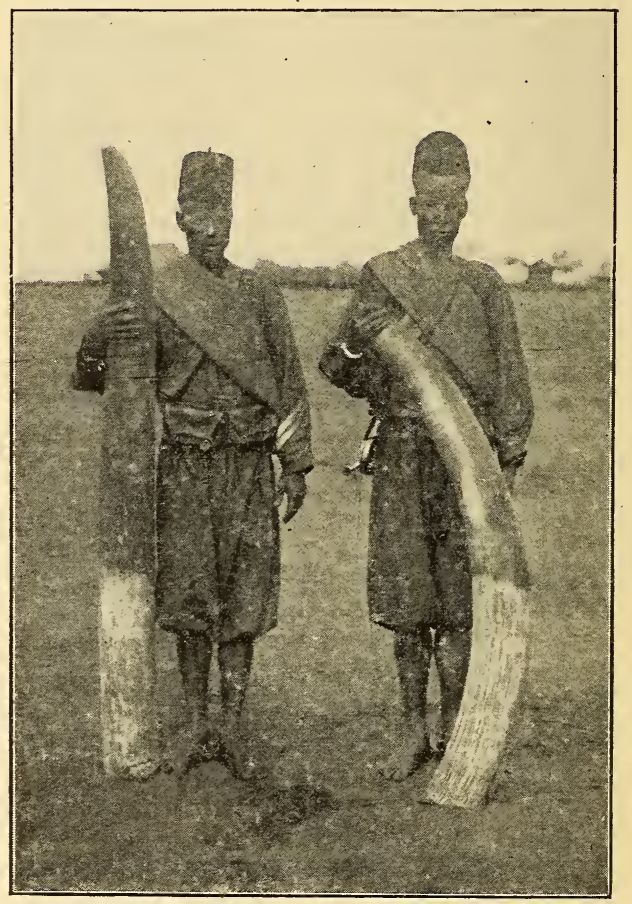

Fig. g6. - DÉFEnses D'un ÉlÉPhant tué par les membres de la Mission Congo-Nil, près de la place du Yé-Yi. Les deux personnages représentés sont des soldats de la, force, publique.

(Photogr. Lemaire.) 
dépouilles animales sont négligeables au point de vue économique et commercial à l'exception de l'ivoire d'éléphant et d'hippopotame. Cette matière constitue encore un article

L'EXPORTATION DE L'IVOIRE

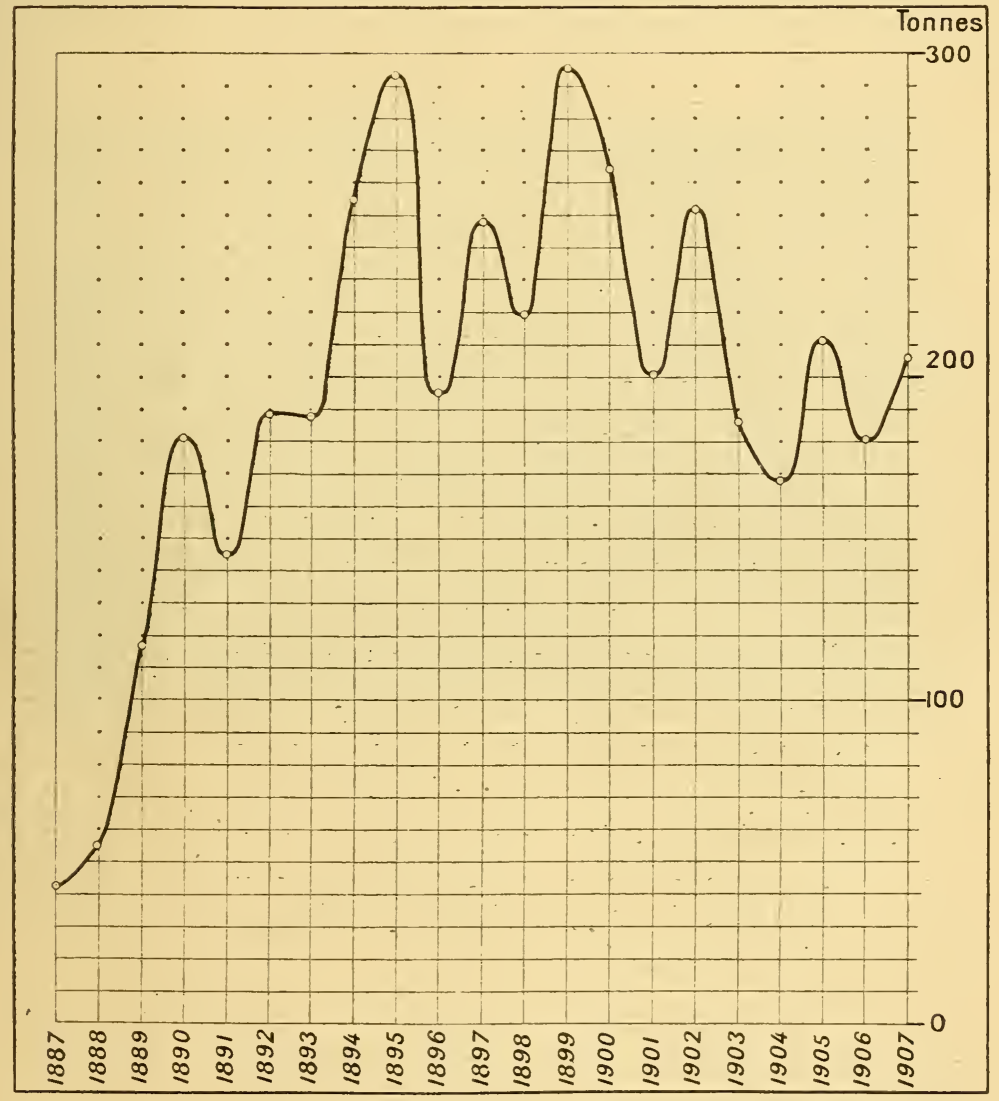

Fig. 97.

(D'après les statistiques officielles)

d'exportation important. Dans un avenir rapproché la production en diminuera au point de devenir tout à fait secondaire. En I906, le Congo a exporté environ i80,00o kilos 
d'ivoire (mort et vivant) pour près de 4,500,000 francs. Il est le premier producteur du monde (total mondial : environ 600 tonnes en 1906 dont les 5/6 originaires d'Afrique). Les trois grands marchés importateurs sont Anvers (327,800 kilos en 1907), Londres (24I,000 kilos) et Liverpool (22,000 kilos).

L'élevage. - Ia mouche tsetsé empêche l'extension du bœuf, qu'elle tue. Il faut donc au gros bétail des soins multiples et constants pour lui permettre de lutter contre des conditions défavorables. Les indigènes de la savane et de la brousse, c'est-à-dire ceux du sud-ouest et plus particulièrement ceux de l'extrême est et nord-est ont pu former des troupeaux de bœufs. Par de grands efforts, l'Européen est parvenu à constituer quelques centres d'élevage qui progressent grâce aux splendides pâturages de la savane (BasCongo, Bas-Kasaï, Manyéma, Rouanda, Katanga). Le but poursuivi est l'amélioration des races indigènes par croisement avec des races importées. Outre l'utilisation comme viande de boucherie, le bœuf présente pour le Congo l'intérêt capital de pouvoir avec avantage remplacer les indigènes dans le portage caravanier et de servir comme animal de trait pour les lourdes charges. En I906, l'État du Congo était propriétaire de 5,000 têtes de gros bétail; à ce chiffre il faut ajouter celui des troupeaux des indigènes et des éleveurs, notamment les 7,000 têtes appartenant à une société d'exploitation du Bas-Congo.

L'importation du cheval, qui, lui aussi, succombe sous la piqûre de la mouche tsetsé, se fait très lentement. On tente des croisements avec le zèure dont la domestication fait l'objet d'essais. L'âne et le mulet appelés à rendre de grands services ne sont pas assez répandus. - Le mouton à poils, du Soudan et le mouton sans laine, la chèvre, le cochon sont assez communs et sont dans de bonnes conditions de déve- 
loppement. -- La basse-cour indigène se réduit à la poule, très abondante d'ailleurs, et s'est enrichie de poules, de canards et de pigeons européens qui croissent bien et rapidement. On mange aussi le chien.

La domestication de l'éléphant et du zèbre est un des points importants de l'activité économique du Congo en

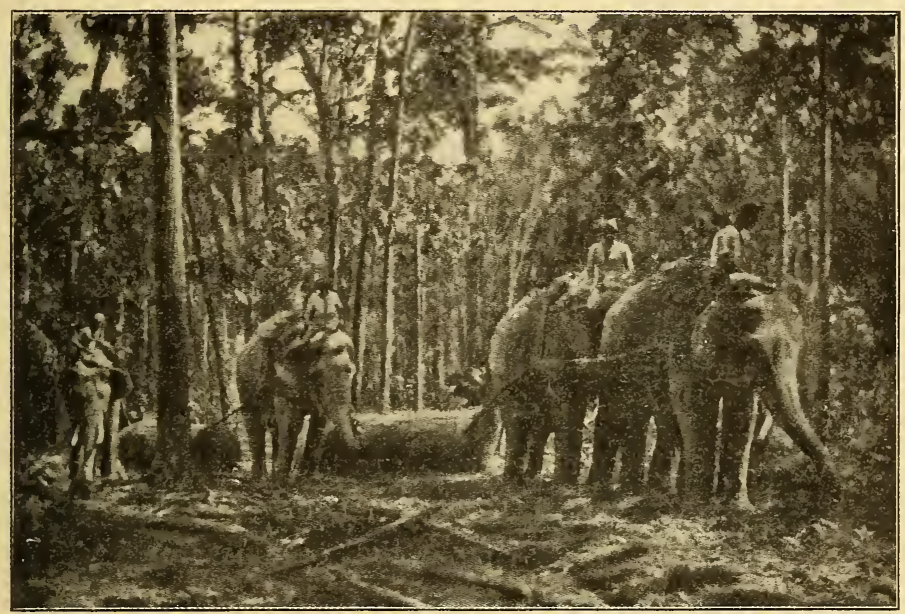

Fig. 98. - Euplor D:S ÉlÉPhunts dans les exploitations forestières aux Indes. Forèt de tecks. (D'après le Bilder-Atlas zur Zoologie der Säugetiere de H. Marshall;Bibliogr. Instit. Leipzig.)

raison des services que ces animaux rendraient dans les transports. C'est à Api, chez les Azandés, à 20 kilomètres au nord de l'Ouellé que les essais de la domestication de l'éléphant se font. Le troupeau ne compte que des individus très jeunes dont les plus âgés n'ont pas 8 ans (à I 5 ans l'éléphant est adulte). Il y a peu de temps, les éléphanteaux d'Api étaient au nombre de 25 mesurant de $\mathrm{I}^{\mathrm{m}} 30$ à $\mathrm{I}^{\mathrm{m}} 72$. Ils travaillaient déjà. Dans le nord le dromadaire et l'autruche pourront aussi, semble-t-il, être employés ou exploités avec avantage. 
Voilà la situation actuelle des territoires de la colonie congolienne au point de vue de l'exploitation forestière et agricole. A celui qui réfléchit et qui songe aux conditions d'étendue et de climat, il apparaît clairement que les deux plus grands problèmes de la mise en valeur et de l'exploitation de la Congolie sont: $I^{\circ}$ la main $d^{\prime}$ 'auvre et $2^{\circ}$ les moyens de transport. Nous allons confirmer cette opinion par l'examen rapide des ressources minérales du pays.

II. - LES GITES MINÉRAUX ET LES ROCHES. -- Les recherches minières au Congo sont encore dans les débuts. La nature du sol et la disposition des couches géologiques (voir plus haut) ont concentré ces recherches sur le pourtour surélevé de la grande plaine du milieu, dans laquelle les terrains anciens sont recouverts d'une épaisse couche de terrains récents et d'alluvions lacustres ou fluviatiles.

Le minerai de fer à l'état de magnétite surtout, d'oligiste ct de limonite est réparti en très grande quantité à peu près partout. Le cuivre surtout, sous forme de malachite en gisements très riches, est très fréquent. L'or existe et a déjà été exploité (en I 906 : 274.5 kilos, soit 851,500 francs). Des filons riches en étain permettent déjà une faible exportation. Enfin l'argent, le nickel, le manganèse, le plomb, le zinc ont été reconnus en différents endroits; la houille aussi, même le diamant. Quant à la pierre exploitable pour la construction, elle existe en assez grande quantité et pourra toujours satisfaire aux besoins futurs du pays : grès rouges et blancs sur toute l'étendue du bassin, schistes formant ceinture aux grès, granits sur le pourtour, calcaires à chaux et calcaires marbres dans le sud-est, le nord-est et l'ouest, argiles en dépôts considérables çà et là. Le sel est abondant, mais localisé. Il est très apprécié des indigènes. La région des grands lacs en possèdent de petits gisements d'origine lacustre dont la production dépasse notablement la consommation 
locale. Certains indigènes de la région oubanghienne et du centre, privés par la nature de chlorure de sodium (sel) le remplacent par un produit auquel ils ont depuis des siècles adapté leur physiologie et qui constitue un poison pour tout organisme non adapté, pour le nôtre par exemple ; il s'agit du chlorure de potassium qui charge les cendres de végétaux par lesquelles ces indigènes remplacent le sel, denrée rare et précieuse, servant souvent d'étalon d'échange.

La région minière par excellence est le Katanga, particulièrement riche en fer et en cuivre (et en sel; moachia). Ces gisements permettront plus tard une exploitation sur une très grande échelle.

Nous avons eu plus haut l'occasion de constater le parti que les indigènes ont tiré des richesses de leur pays, en fer et en cuivre. Ils n'exploitent que les gisements qui affleurent.

En ce qui concerne la mise en valeur des gisements du Katanga, voici des parples autorisées :

" ... c'est que de récentes explorations n'ont fait que montrer davantage leur grande importance et la possibilité de leur exploitation industrielle, lorsque le développement des moyens de communication le permettra. M. Cornet a également décrit de nombreuses mines de fer qui peuvent passer, dit-il, pour les plus importantes du monde. Si dans l'état actuel, ces mines sont sans valeur au point de vue industriel, les choses n'en resteront pas toujours là et il est du plus haut intérêt de savoir qu'il existe là, à proximité d'une région que l'exploitation du cuivre rendra certainement industrielle, des amas énormes de fer dont nul ne saurait prévoir l'avenir »(Stainier).

L'on assure que dès l'établissement d'une ligne ferrée, le Katanga fournira 100 tonnes de cuivre par mois et l'on prévoit même une production de 100,000 tonnes par an. La quantité de combustible nécessaire pour le traitement du minerai étant considérable, on compte la réduire au minimum en adoptant le traitement électrique rendu aisé par la captation de l'énergie en houille blanche des chutes de la Loufira et de la 
Loualaba. La force de ces chutes est évaluée à I 50,000 chevaux.

La double question de la main d'œuvre et des voies de communication se pose ici, plus impérieusement encore que pour l'exploitation agricole, en raison de l'éloignement du Katanga. Il apparaît comme évident que cette exploitation minière ne pourra être engagée sur une grande échelle que plus tard, lorsque la mise en valeur agricole et forestière, véritable fond économique de la colonie, aura créé et alimentera déjà un réseau de voies de communication. C'est alors seulement que sera praticable et rémunérateur le transport des matières minérales pondéreuses et encombrantes entre, l'arrière-pays minıer et la côte.

III. - Le mouvement commercial. - En i887, l'État du Congo produisait et exportait pour $1,980,000$ francs de marchandises (caoutchouc, II 7,000 francs; ivoire 795,500 fr.; noix palmiste, 79I,000 francs; huile de palme, 462,500 francs; copal, arachides et divers, I4,500 francs). Vingt ans plus tard, en 1906, les exportations se chiffraient par 58,278,000 francs (caoutchouc, 48,489,000 francs; ivoire, 4,455,000 francs ; noix palmistes, I,469,000 francs ; huile de palme, $1,197,000$ francs ; copal blanc, I,086,000 francs; or, 85I,000 francs; cacao, 563,000 francs; café, 75,000 francs ; riz, étain, peaux, arachides, minerai de cuivre et divers, 93,000 francs).

En I893, première année où la statistique des importations se fit, l'Etat du Congo importait et consommait pour 9, 1 75,000 francs. Treize ans après, en 1906, ce chiffre montait à 2 I $, 478,000$ francs.

Nous envisageons là ce que le Congo produit et exporte, ce qu'il importe et consomme, abstraction faite du transit. C'est le commerce dit spécial de l'Etat du Congo qui, en I906, se chiffrait donc par 58,278,000 (exp.) et 2 I,478,000 (imp.) ce qui donne 79,756,000 francs. En I893, ce commerce 
LE COMNERCE SPÉCIAL DU CONGO (EN MILLIONS DE FRANCS)

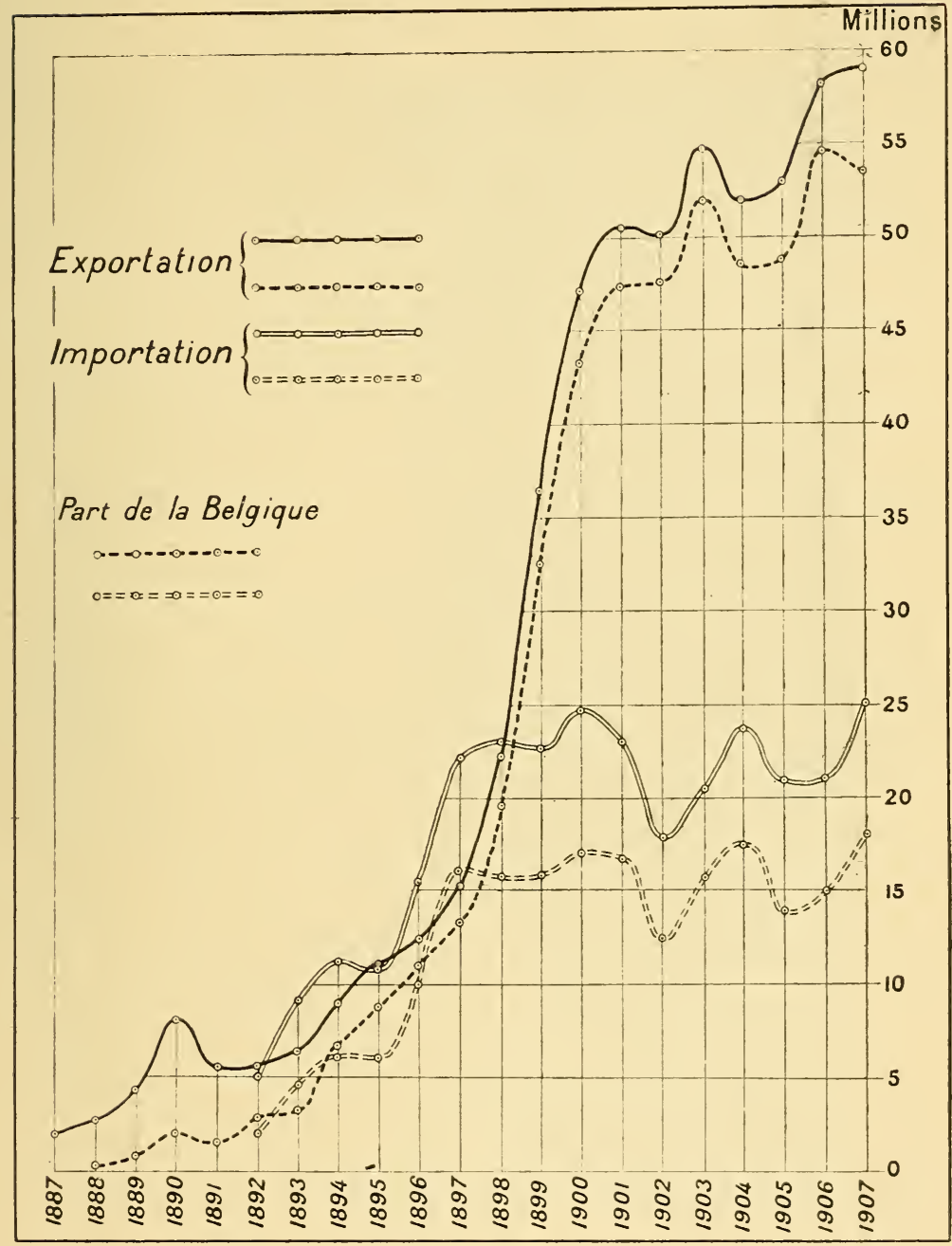

Fig. 99.

D'après les statistiques officielles.) 
était de 6,206,000 (exp.) plus 9,I75,000 (imp.) ce qui donne I 5,88I,000 francs. Il a donc plus que quintuplé en I 3 ans.

Les exportations au compte du gouvernement de la colonie, de sociétés concessionnaires et aussi de particuliers, se font en presque totalité à destination de la Belgique par la voie de Matadi-Boma-Anvers. De même, la Belgique est le principal importateur. Comme tout pays neuf, producteur de matière première, le Congo importe surtout des objets fabriqués, d'usages divers : ${ }^{\circ}$ objets d'échange commercial, tels que tissus de coton, de laine, de toile, de soie, fil de laiton, perles, bibelots, quincaillerie, vieilles armes: $2^{\circ}$ machines et outils pour les chemins de fer, la navigation, l'exploitation agricole et minière, la construction, etc... A l'importation figurent aussi les matières alimentaires en conserves à destination de la population blanche.

IV. - Les voies de COMmunicAtion. - Les administrateurs de la colonie léopoldienne ont de tout temps compris la nécessité d'un réseau de voies de communication, permettant une pénétration aisée et un draînage rapide et peu coûteux des produits. Dès l'origine de la colonie, les efforts furent dirigés dans ce sens et poursuivis sans relâche. "Sans chemins de fer, le Congo ne vaut pas un penny " avait dit Stanley.

La création d'un chemin de fer d'escalade des contreforts africains percés en cascades par les eaux congoliennes, devait permettre l'utilisation progressive d'un réseau naturel de I 5,000 kilomètres de voies d'eau. La création et l'entretien de routes et de chemins de fer intérieurs devaient réduire les difficultés et aboutir au résultat commercial étonnant que nous avons signalé plus haut et qui est unique dans l'histoire de l'exploitation coloniale. 


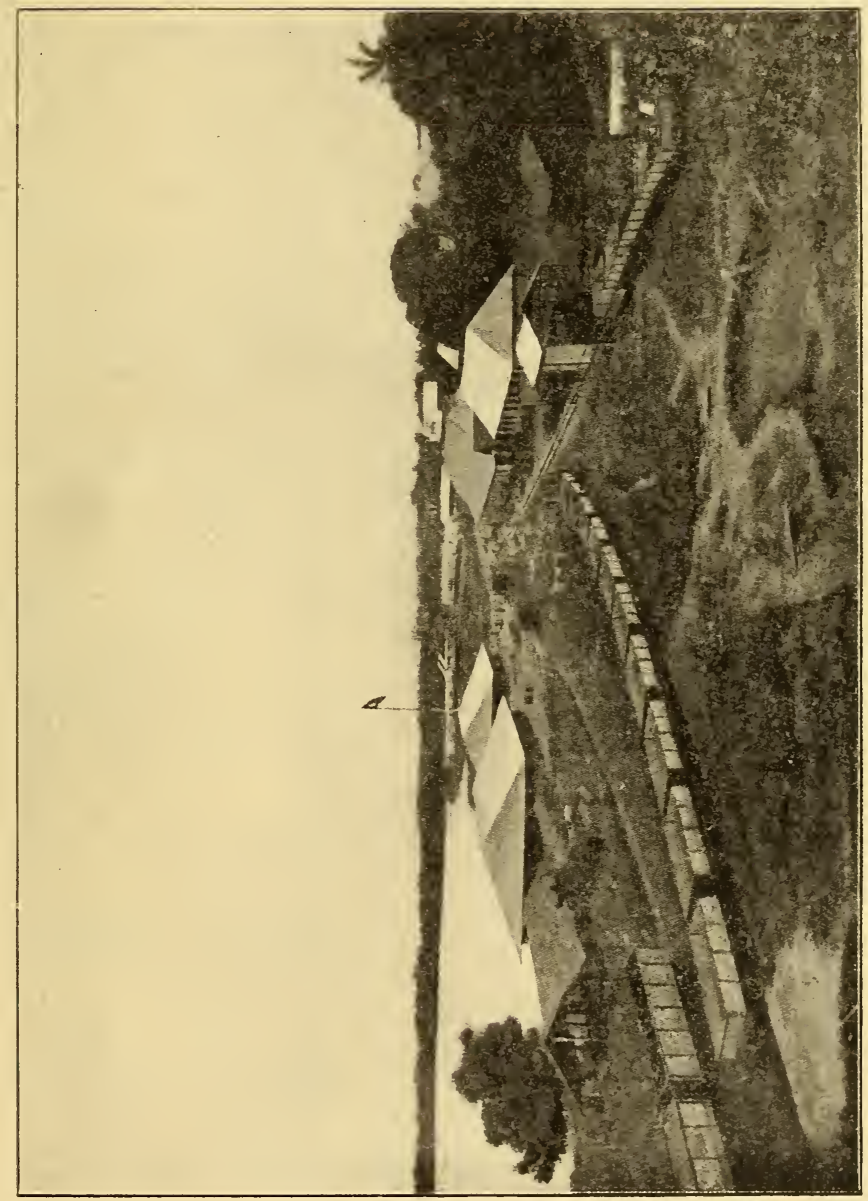

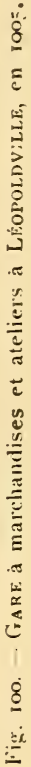


Comment on arrive au Congo. - I. Par l'embouchure du Congo. - $I^{\circ}$ Ligne Anvers-Las Palmas (Canaries)-Banana-Boma-Matadi, (Compagnie belge maritime du Congo), 9,000 kilomètres, départ toutes les trois semaines; durée : 18 à 20 jours; - $2^{\circ}$ Ligne Lisbonne-Cabinda-San Antonio do Zaïre et au delà (Compagnie portugaise), départ tous les quinze jours, durée : 20 à 22 jours; - $3^{\circ}$ Ligne Le HavreBordeaux-Banana-Boma-Matadi, (Compagnie française), départ une fois par mois; -- $4^{\circ}$ Ligne Anvers-CabindaBanana-Boma-Matadi, (Compagnie hollandaise), départ une fois par mois; $-5^{\circ}$ Ligne Liverpool à toute la côte occidentale de l'Afrique (Compagnie anglaise), départ toutes les semaines.

II. Par la voie du Nil. - I.ignes française, allemande ou anglaise à Alexandrie; remontée de la vallée nilienne en bateau et chemin de fer (Alexandrie à Lado : 35 jours).

III. Par l'Océan Indien. - I $^{\circ}$ Lignes anglaise ou allemande à Mombasa. Traversée des territoires anglais en 30 à 35 jours (voir carte). $2^{\circ}$ Lignes anglaise ou allemande à Dar-es-Salaam. Traversée des territoires allemands. Route abandonnée.

IV. Par Capetown et le Sud-africain, jusqu'à Broken Hill (voir carte).

La voie absorbant presque tout le trafic des voyageurs et des marchandises est celle d'Anvers-Boma-Matadi.

Comment on circule au Congo.- Une fois que le chemin de fer du Bas-Congo, contournant les chutes Livingstone, de Matadi à Léopoldville, fut construit et mis en exploitation (juillet I898), tous les produits de l'intérieur pouvaient arriver à la mer et être versés sur le marché du monde. C'est de cette époque que date l'essor commercial vrai du Congo (voyez les diagrammes). Léopoldville et Matadi, l'une comme point de concentration et centre d'équi- 
L'Afrique politique. -- Les voies de COMMUUNication

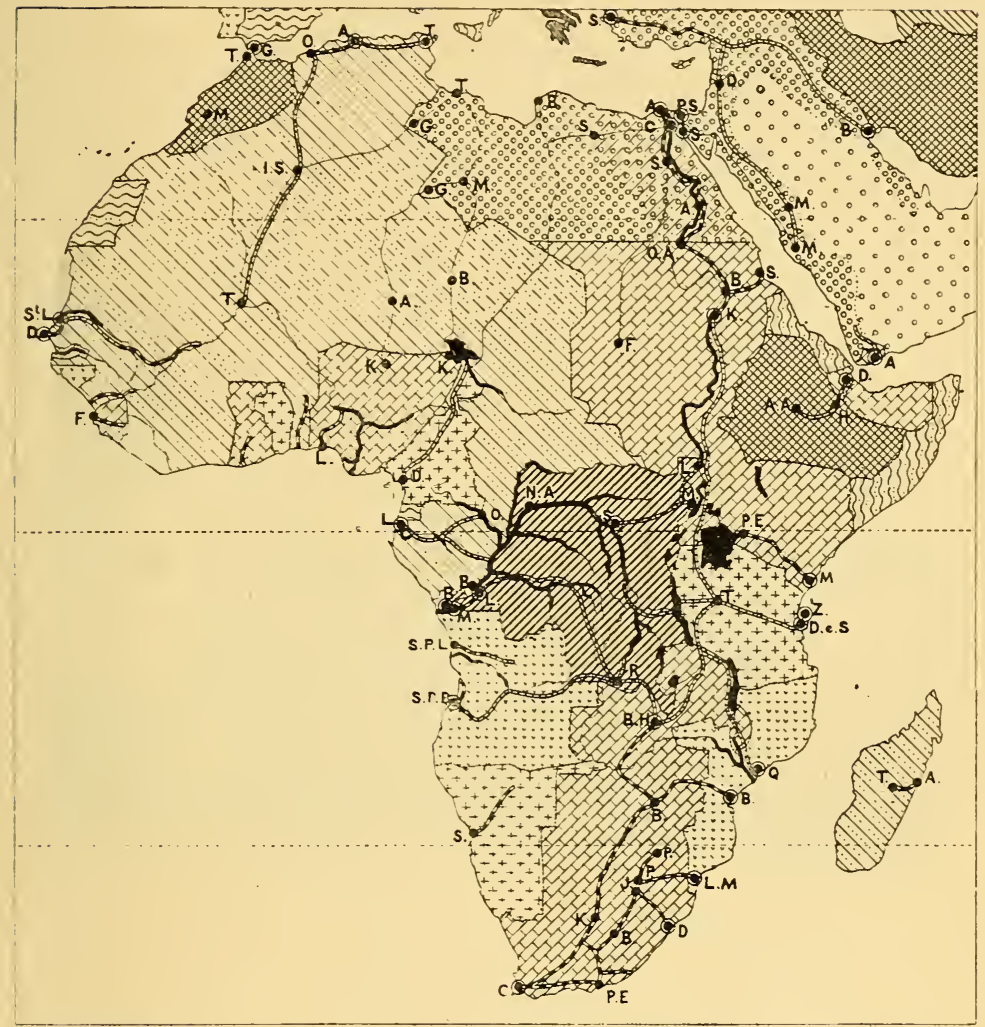

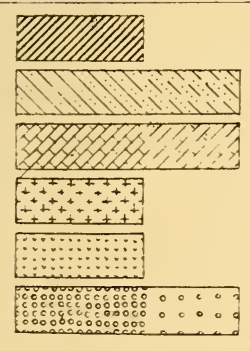

Fig. ror.
Possessions belges.

Possessions françaises

Possess. britanniques (et territ. d'infl. britan.)

Possessions allemandes.

Possessions portugaises.

Possessions turques iet territoires nominalement possédès par la Turquie.)
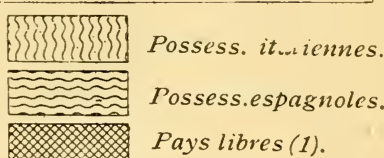

Possess.espagnoles. Pays libres (1).

Chemins de fer en exploitation.

Chemins de fer projetés.

Cours d'eau nari. gables.

Routes decararane's.

(1) Par erreur, la république de Libéria n'est pas indiquèe comme Etat libre. (Petit espace en blanc à la côte de Guinèe) - (Voir texte p. 23.) 
libre de toutes les voies fluviales du Congo, l'autre comme seul point de contact maritime et d'éjection au dehors des produits congoliens, sont les deux points vitaux du commerce du pays. Boma, sur l'estuaire, a acquis comme centre capital de l'adrinistration, un développement considérable.Conserve-

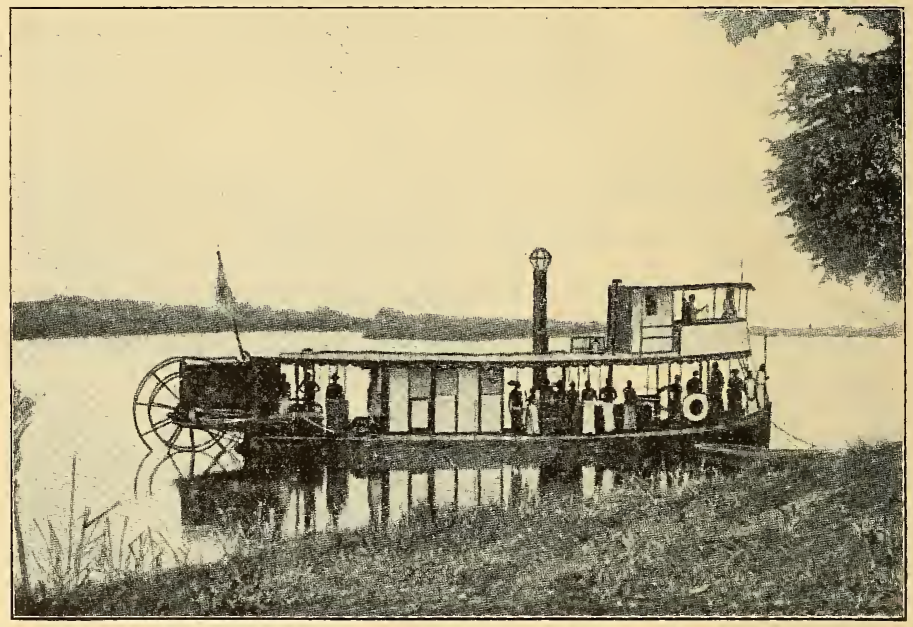

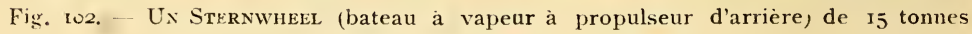
(le Baron Dhanis, sur le Loualaba)

ra-t-il cette importance ? Cela est peu probable, car les voies de communications se perfectionnant, l'administration centrale trouvera profit de se déplacer vers l'intérieur. A Banana semble réservée une prospérité commerciale considérable, grâce à sa belle rade hospitalière.

En amont des cataractes, la navigation fluviale se fait à l'aide de bateaux plats à propulseurs d'arrière ou de côté, mus à la vapeur, à moins que les rivières ne présentent pas une profondeur suffisante. Dans ce cas on emploie d'assez grandes allèges plates, des barques européennes ou des pirogues indigènes. Les principaux ports fluviaux, Léopoldville, 
Kinchasa, Ponthierville, possèdent des chantiers de montage qui ont livré à la circulation environ quatre-vingts petits steamers. La résolution du problème des voies de transport se poursuit par l'établissement de chemins de fer d'exploitation locale, de voies ferrées contournant les chutes et rapides $\mathrm{du}$ haut-fleuve, par l'étude de la ligne joignant le Nil au Congo, le Katanga à Léopoldville, etc... (Voir sur la carte : ligne vicinale du Mayoumbé ; ligne de Stanleyville à Ponthierville; ligne, actuellement en construction, de Kindou à à Kongolo qui permettra l'accès au plus haut bief navigable du Loualaba.)

Des efforts considérables ont été faits pour compléter le réseau des voies fluviales et ferrées par un réseau de routes de roulage (route Congo-Nil de Bouta à Redjaf (I) et route Sankourou-Loualaba de Pania à Bouli), mais le résultat obtenu ne concorde pas avec l'effort fourni. On ne voit guère sur ces routes que des caravanes de porteurs. Les routes de portage proprement dites, assez nombreuses, sont plutôt des sentiers plus ou moins larges le long desquels s'acheminent les caravanes de porteurs et dont nous avons eu l'occasion de nous entretenir à propos du commerce des indigènes.

Le service des postes est organisé sur toute l'étendue de la colonie. Celui des télégraphe et téléphone fonctionne entre quelques points : de Boma à Coquilhatville (ligne télégraphique suivant le Congo et desservant les stations intermédiaires) ; de Kasongo sur la Loualaba à Ouvira sur le Tanganika (ligne télégraphique); de Stanleyville à Ponthierville (ligne télégraphique).

Dans un avenir assez rapproché, les chemins de fer allemands et anglais de l'est et surtout la ligne anglaise transafricaine Cap-Caire (voir cartes) prendront une très grande

(1) En avril 190?, trois voitures automobiles faisaient le service de Bouta vers l'Ouellé. 
importance dans le commerce de la partie orientale de notre colonie, depuis les pays du Nil jusqu'aux confins de la Zambézie.

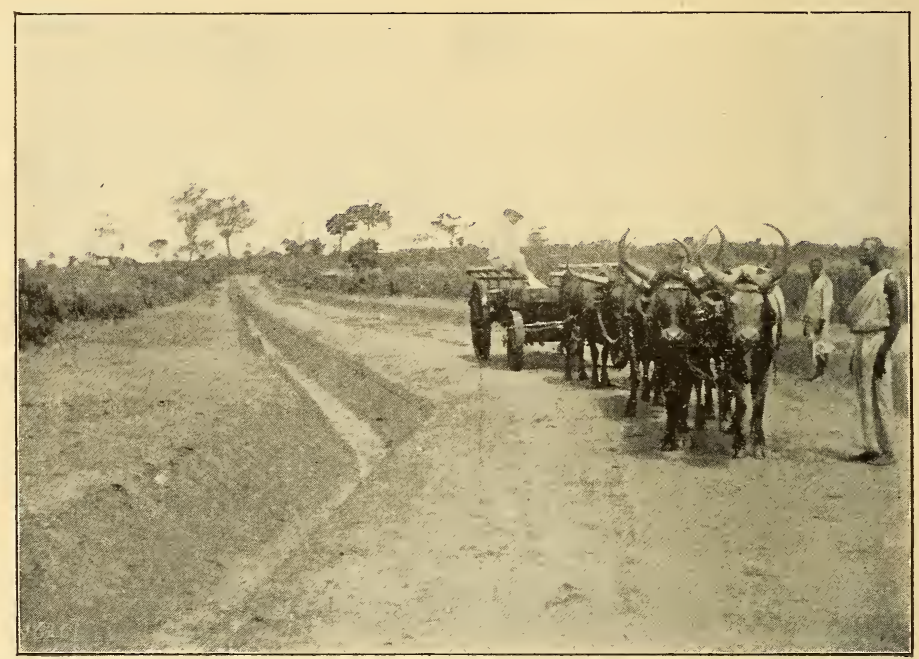

Fig. 103. - LA ROUTE DE ROULAGE près de Lousambo. (Photogr. Verdick.)

V.- LA MAIN D'EUVRE.- La traite a été justement abolie; mais au Congo subsiste encore cette forme indigène assez atténuée et d'ailleurs indéracinable ex abrupto de l'esclavage domestique. Dans les débuts de la pénétration, le blanc a eu recours à l'engagement de travailleurs étrangers originaires en grande partie de Zanzibar et des côtes de Guinée, car les indigènes se montraient méfiants et réfractaires à tout travail. Les efforts de Stanley en I880 et I88 I et ceux de tous ses successeurs aboutirent à la création parmi les indigènes de la côte de la profession de porteur. En I 893 déjà, on estimait à 50,000 le nombre des noirs circulant sur la route des cataractes. Depuis, l'indigène de l'intérieur et de la côte s'est familiarisé avec le travail du portage, de la culture, de la 
forêt, du chantier et de l'atelier de construction. Les Congoliens sont portefaix ou pagayeurs, ouvriers des champs ou de l'atelier ; ils sont cultivateurs, jardiniers, bûcherons, machinistes, poseurs de voies ; ils sont employés ou magasiniers, cuisiniers ou hommes de peine, guides ou domestiques (boys). Il $\mathrm{y}$ a certaines régions où tous ces travailleurs se recrutent sans peine, non pas qu'ils soient artisans accomplis, ils ont tout à apprendre, sont en général de bonne volonté et le restent s'ils sont traités avec égard, bonté $\approx$ justice. Leurs salaires sont d'ailleurs bas. En moyenne fr. I.50 par jour à la côte ; le salaire descend, dans l'intérieur, à quelques pièces d'étoffes de 5 francs comme mensualité. Nous avons vu plus haut que l'impôt se payait à l'État en heures de travail (40 heures par mois). Ajoutons à cela l'entraînement aux travaux manuels des soldats de la force publique. Nous nous expliquons ainsi comment l'État a pu, à très bon compte, créer de très vastes plantations et s'assurer un revenu considérable.

En outre, les missions catholiques s'occupent très activement de l'éducation professionelle du nègre.

En I897, M. Wauters écrivait :

« La question de la main-d'œuvre semble donc en voie de solution. Les résultats obtenus en si peu d'années permettent de bien augurer de l'avenir. On peut entrevoir le jour où il deviendra inutile d'importer des travailleurs exotiques, où les bras s'offriront sur tous les points du territoire et où, grâce à l'organisation du travail sur les bases de la liberté, chacun des pauvres villages d'aujourd'hui sera transformé en un centre de production et de richesse. $)$

Nous avons souligné quelques mots à cause de leur vérité et de leur actualité. En effet, dans l'élaboration par le parlement belge du régime de la colonie, l'étude de la situation $\mathrm{du}$ travailleur noir prendra son importance vraie et aboutira à une solution qui marquera dans l'histoire de la colonie belge. 


\section{Le même auteur disait :}

«On a vanté les avantages qu'offrirait, autant comme procédé de colonisation que comme moyen de se procurer une main-d'œuvre régulière et à bon marché, la déportation des individus condamnés dans la mère-patrie, les vagabonds et les malfaiteurs. Ce système qui a fait ses preuves au Brésil et surtout en Australie ajoutons en Nouvelle Calédonie, où la France a souvent des difficultés - a trouvé des partisans en Belgique... Cependant, on ne pourrait le préconiser; ce n'est pas en mettant les indigènes en contact avec le rebut de la société européenne, avec des êtres lâches, paresseux et corrompus, cueillis sur le pavé de nos grandes villes, qu'on arrivera à rehausser le niveau moral et intellectuel des races africaines : la présence de pareils déchets serait extrêmement nuisible au développement de la colonie. 》 


\section{L'AVENIR DE LA COLONIE.}

La marche ascendante de la puissance commerciale de la Congolie est un exemple unique de rapidité dans l'histoire de l'exploitation coloniale. Il a été prouvé que le milieu naturel centrafricain est d'une richesse extrême et que la population nègre, contrairement à beaucoup d'affirmations téméraires, est fort apte au travail et s'offre spontanément au progrès social. Nous nous sommes rendu compte des problèmes et des difficultés résolues ou à résoudre: la main d'cuvre et les communications. Nous avons clairement entrevu que la solution du premier de ces problèmes comporte en elle la solution du second et dépend elle-même de l'organisation du travail sur les bases de la liberté et du recrutement des éléments colonisateurs blancs.

En tant que producteur, le bassin du Congo apparaît comme un des pays les plus féconds.

De tout cela, et sous la condition expresse que les sacrifices financiers nécessaires soient consentis par la jeune métropole, nous inférons, pour un avenir peu lointain, au rôle prépondérant de la colonie congolienne dans l'économie de l'Afrique et à l'importance de sa place sur le marché du monde, au plus grand profit commun du noir et du blanc.

A côté de ces problèmes d'ordre économique s'en dressent d'autres d'ordre éducatif. Le colon agriculteur, ouvrier ou fonctionnaire et l'indigène demandent à être "professionnellement armés dans l'œuvre d'aménagement du pays que l'on 
attend d'eux. L'éducation agricole ou technique du colon s'ébauchera ici, avant son départ et se parfera en des fermesmodèles ou des chantiers-écoles au Congo (I). Pour l'indigène, les écoles professionnelles créées par l'Etat à Boma, Léopoldville et Stanleyville, et les écoles fondées par les missionnaires seront développées, de nouveaux et nombreux établissements scolaires seront organisés.

Sur toute l'étendue de la colonie, des écoles primaires à tendances professionnelle et technique s'imposent, mais, au préalable, des instituteurs indigènes devront être formés danr dés écoles normales à créer dans les principaux centres du Congo. D'une part la psychologie du nègre, d'autre part la prospérité de l'école normale du Gabon et de l'école pour adultes illettrés, toutes deux à Libreville (Congo français) nous sont des garanties de l'empressement de l'indigène à s'instruire. Que des enfants nègres viennent prendre contact

(1) Au moment de mettre sous presse, notre attention est attirée par un arrêté ministériel promulgué le 30 mai dernier :

Service de l'agriculture. - En vue de mettre les agents du service de l'agriculture de la colonie à même de recevoir un enseignement théorique et pratique, il est créé : $1^{\circ}$ à Laeken, une ferme modèle; $2^{\circ}$ à Laeken et à Tervueren, des établissements de culture maraîchère et fruitière.

L'enseignement porte sur les matières suivantes: $1^{\circ}$ notions d'anatomie, de physiologie et de zootechnie des animaux domestiques; $2^{\circ}$ alimentation, hygiène, maladies et reproduction du bétail; $3^{\circ}$ dressage $d u$ bétail à la traction d'instruments aratoires et de véhicules; $4^{\circ}$ étude et mise à l'essai des instruments et des machines agricoles destinées au Congo; $5^{\circ}$ aviculture; $6^{\circ}$ culture maraîchère et fruitière appropriée aux conditions spéciales des régions tropicales; préparation et fumure des terres; multiplication des légumes et des plantes fruitières; production et sélection de graines potagères destinées au Congo.

Les institutions créées par le présent arrèté formeront des sections professionnelles de l'Ecole mondiale de Tervueren. 
avec notre civilisation et fréquenter nos écoles chez nous, que des pédagogues aidés par ceux qui connaissent le mieux la psychologie du noir aillent au Congo organiser des écoles normales, pépinières d'instituteurs noirs pour enfants noirs. Il $y$ a là un genre d'importations et d'exportations aussi nécessaire à la métropole qu'à la colonie.

Enfin le Congo, si sombre, il y a 25 ans, nous est devenu mieux connu. D'année en année, de jour en jour, de meilleures conditions de séjour s'y affirment pour l'Européen. La mortalité diminue é tomberait beaucoup plus bas, s'il était possible aux agents coloniaux de vivre dans un milieu familial. Les missionnaires protestants, accompagnés de leurs épouses, font de très longs séjours dans les colonies équatoriales et les missionnaires catholiques, en 1907, demandaient que " dans l'intérêt des fonctionnaires et agents, dans l'intérêt de l'État luimêtene qui verrait la qualité de ses agents s'améliorer considérablement, le gouvernement encourageât de plus en plus ses tonctionnaıres à renir au Congo avec leur femme ». Des enquêtes fort intéressantes ont mis en évidence que l'installation des familles blanches aurait comme conséquence une certaine décentralisation, une démilitarisation et une moralisation très effectives qui permettraient au colon, fonctionnaire ou non, de vivre dans des conditions plus normales, meilleures pour lui-même et aussi pour l'indigène dont le souci dominant est de vivre à l'instar du blanc.

L'installation volontaire et la colonisation libre par des familles de colons européens avec l'aide de l'État, feront éclore de nombreux et vigoureux centres agricoles. La voie réritable de la colonisation sera fixée moins dans l'exploitation des ressources minérales, très considérables, sans doute, que dans la mise en valeur par des colons propriétaires agriculteurs européens et indigènes - et l'on sait que les nègres sont d'excellents cultivateurs - d'un fond inépuisable de richesse végétale. 



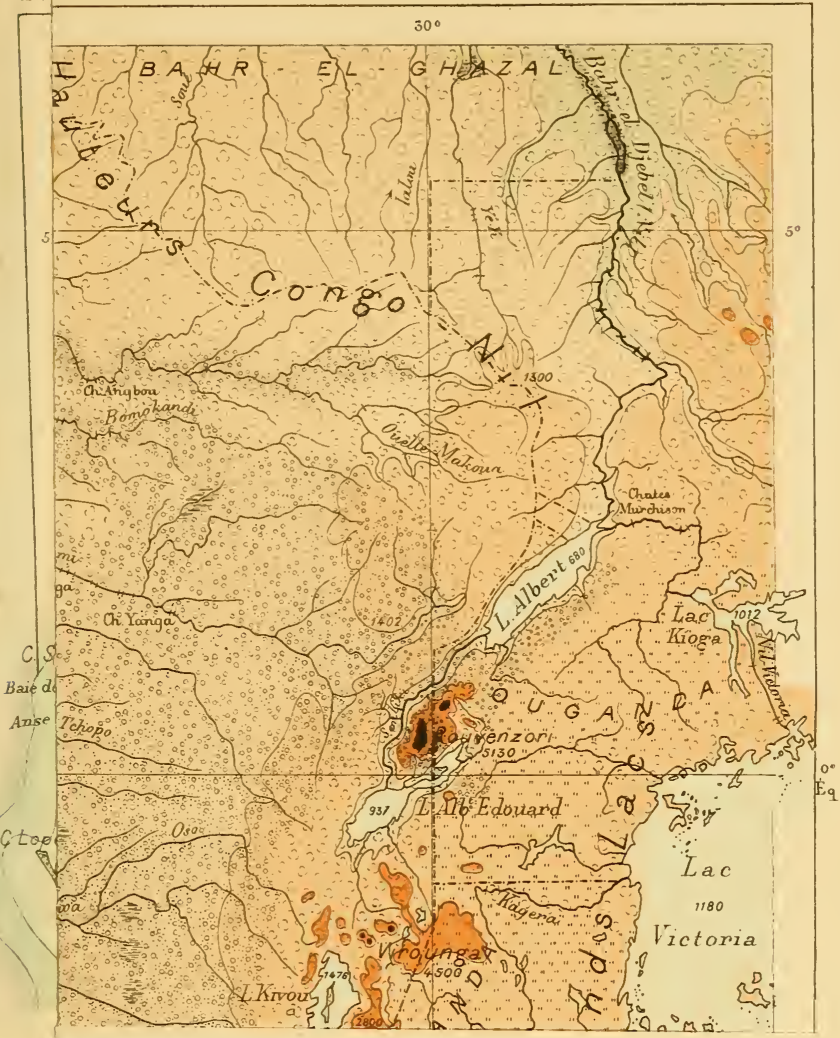





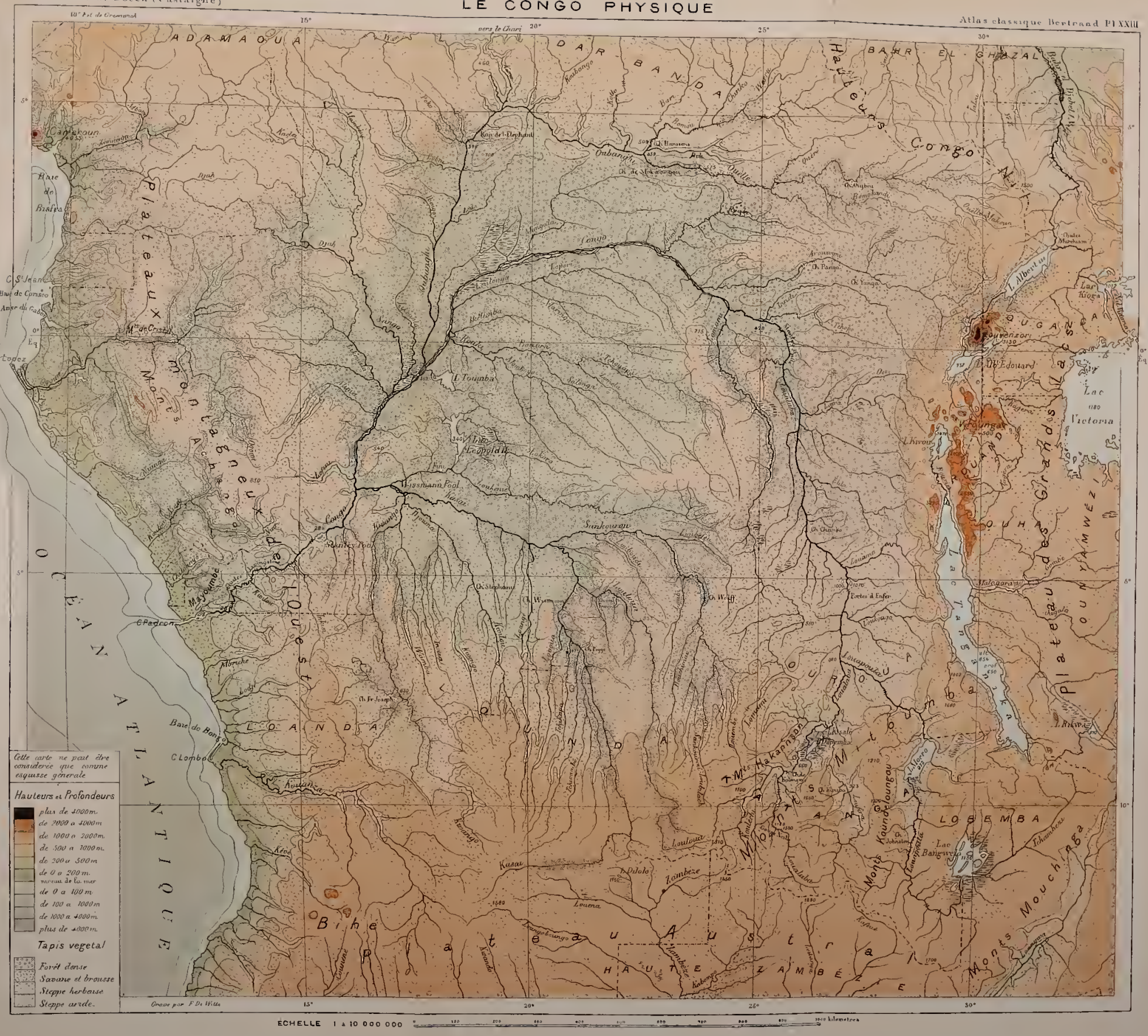





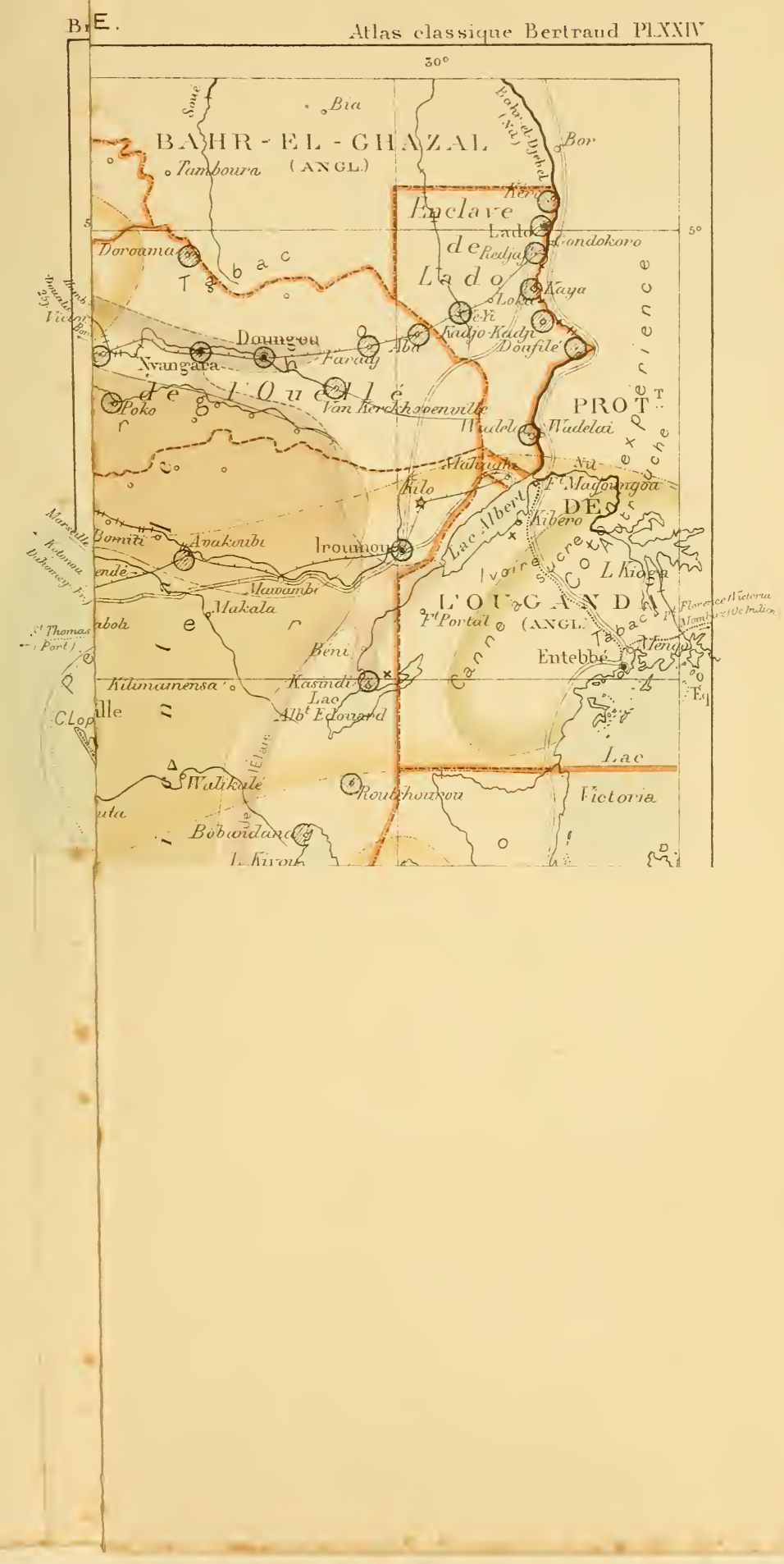









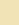


$0^{0}(0 .+1,1 \div-20$

a 


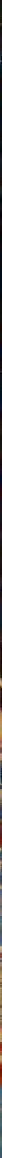
$y^{2}-1$

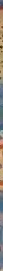

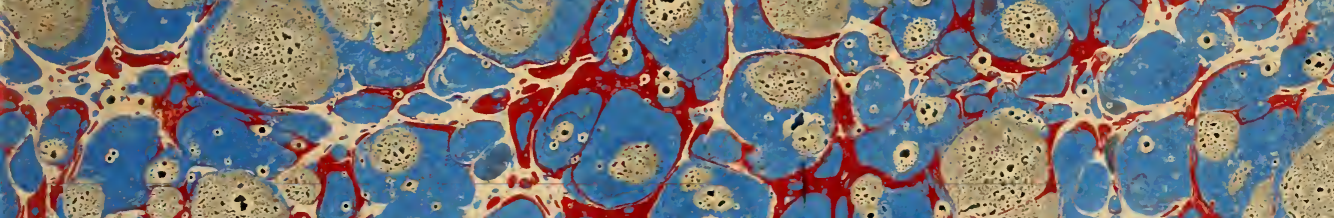

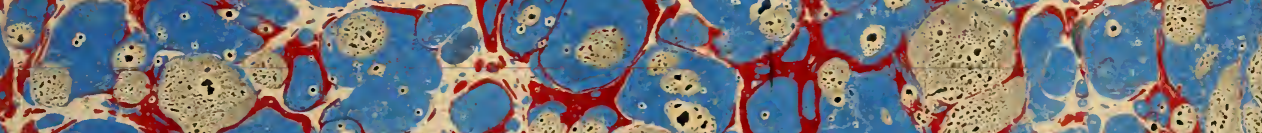
50.5020 .50

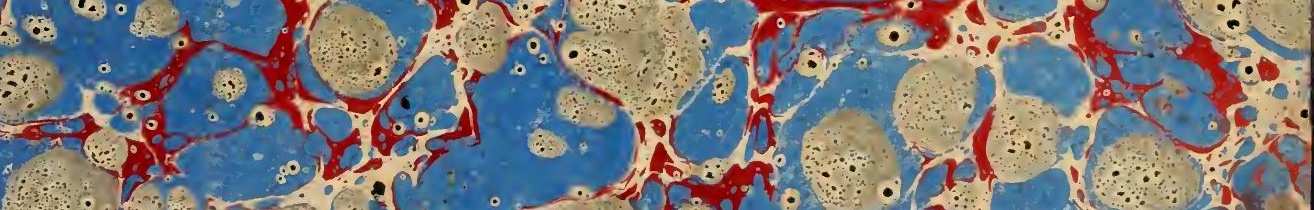

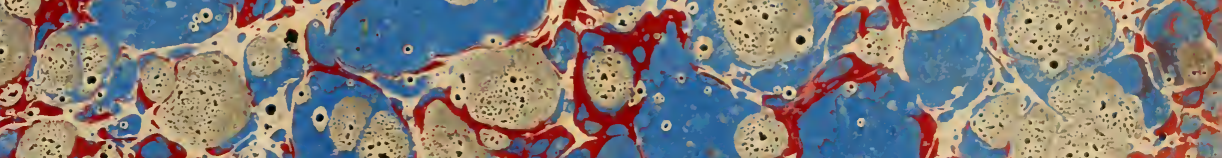




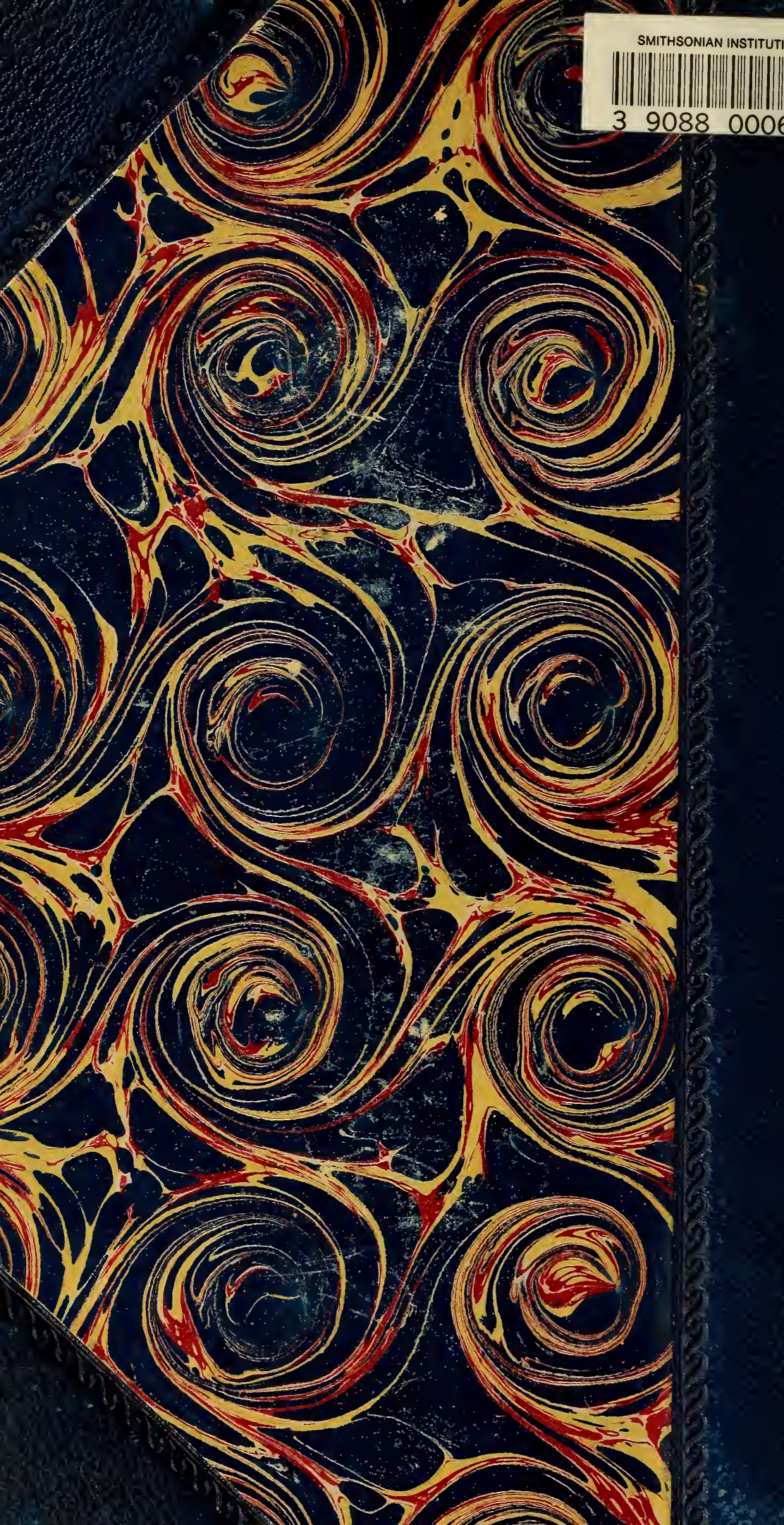

